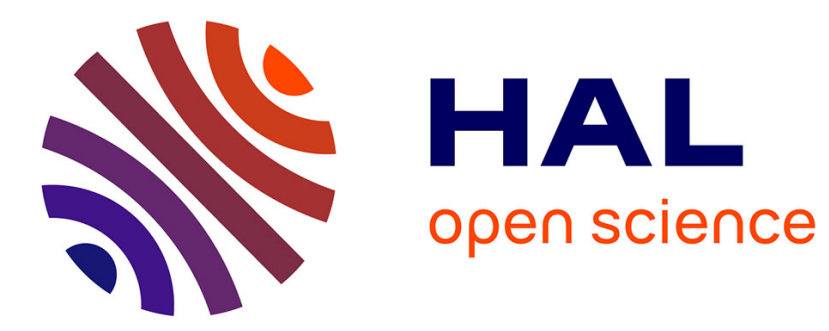

\title{
A Tutorial on Well-Composedness
}

Nicolas Boutry, Thierry Géraud, Laurent Najman

\section{To cite this version:}

Nicolas Boutry, Thierry Géraud, Laurent Najman. A Tutorial on Well-Composedness. Journal of Mathematical Imaging and Vision, 2018, 60 (3), pp.443-478. 10.1007/s10851-017-0769-6 . hal01609892v2

\section{HAL Id: hal-01609892 \\ https://hal.science/hal-01609892v2}

Submitted on 12 Oct 2017

HAL is a multi-disciplinary open access archive for the deposit and dissemination of scientific research documents, whether they are published or not. The documents may come from teaching and research institutions in France or abroad, or from public or private research centers.
L'archive ouverte pluridisciplinaire HAL, est destinée au dépôt et à la diffusion de documents scientifiques de niveau recherche, publiés ou non, émanant des établissements d'enseignement et de recherche français ou étrangers, des laboratoires publics ou privés. 


\title{
A Tutorial on Well-Composedness
}

\author{
Nicolas Boutry · Thierry Géraud · Laurent Najman
}

Received: date / Accepted: date

\begin{abstract}
Due to digitization, usual discrete signals generally present topological paradoxes, such as the connectivity paradoxes of Rosenfeld. To get rid of those paradoxes, and to restore some topological properties to the objects contained in the image, like manifoldness, Latecki proposed a new class of images, called wellcomposed images, with no topological issues. Furthermore, well-composed images have some other interesting properties: for example, the Euler number is locally computable, boundaries of objects separate background from foreground, the tree of shapes is well-defined, and so on. Last, but not the least, some recent works in mathematical morphology have shown that very nice practical results can be obtained thanks to well-composed images. Believing in its prime importance in digital topology, we then propose this state-of-the-art of well-composedness, summarizing its different flavours, the different methods existing to produce well-composed signals, and the various topics that are related to well-composedness.
\end{abstract}

Keywords Well-composedness · Digital topology · Mathematical morphology · Critical configurations · Discrete surfaces · Manifolds

\section{Nicolas Boutry}

EPITA Research and Development Laboratory (LRDE)

14-16 rue Voltaire, FR-94276 Le Kremlin-Bicêtre, France

E-mail: nicolas.boutry@lrde.epita.fr

Thierry Géraud

EPITA Research and Development Laboratory (LRDE)

14-16 rue Voltaire, FR-94276 Le Kremlin-Bicêtre, France

E-mail: thierry.geraud@lrde.epita.fr

Laurent Najman

Université Paris-Est, LIGM, Équipe A3SI, ESIEE

E-mail: l.najman@esiee.fr

\section{Introduction}

In 1979, Rosenfeld [148] studied basic topological properties of digital images that he called digital topology. This work was completed in 90] by Kong and himself. However, Rosenfeld's framework needs to use a dual pair of connectivities to get rid of connectivity paradoxes, leading then to ambiguities: depending on the chosen dual pair of connectivities, the results are not always the same, even for elementary algorithms such as object counting. To overcome this problem, Latecki et al. [104] introduced in 1995 2D sets free from topological paradoxes, called well-composed sets: the connected components of these sets or of their complement do not depend on the chosen connectivity. Observing that the natural extension of this definition is not strong enough in 3D, Latecki et al [100] proposed in 1997 a new definition of well-composedness for 3D sets: a set is said well-composed iff the boundary of its continuous analog is a 2-manifold. In parallel, Wang and Battacharya [183] proposed an extension of 2D well-composedness to arbitrary grids. In 1998, Stelldinger proposed in his book 168 a definition of well-composedness on polytopal complexes, but this definition seems to be illdefined. Fifteen years passed before Najman and Géraud [127] introduced in 2013 the definition of well-composedness in Alexandrov spaces based on discrete surfaces; this definition works in $n$-D and can be applied to cubical complexes defined on Khalimsky grids [88]. Finally, well-composedness on arbitrary grids has been extended to $n$-D in 2016 by N. Boutry [26] in his Ph.D. thesis.

To avoid ambiguities among these different flavors of well-composednesses, we need to precise the terminology. In this paper, well-composedness such that the 


\begin{tabular}{|c|c|c|c|c|c|c|c|c|c|}
\hline 2D: & $\begin{array}{c}\text { EWC } \\
1995[104\end{array}$ & $\Leftrightarrow$ & DWC & $\Leftrightarrow$ & AWC & $\Leftrightarrow$ & CWC & $\stackrel{?}{\leftrightarrow}$ & $\begin{array}{c}\text { AGWC } \\
1997[183\end{array}$ \\
\hline 3D: & EWC & $\Leftarrow$ & DWC & $\Leftrightarrow$ & AWC & $\Leftrightarrow$ & $\begin{array}{c}\text { CWC } \\
1997[100\end{array}$ & $\stackrel{?}{\leftrightarrow}$ & $\begin{array}{l}\text { AGWC } \\
2016[26\end{array}$ \\
\hline$n$-D: & $\begin{array}{c}\text { EWC } \\
2015[29]\end{array}$ & $\Leftarrow$ & $\begin{array}{c}\text { DWC } \\
2015[29]\end{array}$ & $\stackrel{?}{\leftrightarrow}$ & $\begin{array}{c}\text { AWC } \\
2013[127]\end{array}$ & $\stackrel{?}{\leftrightarrow}$ & $\begin{array}{c}\text { CWC } \\
2000[102\end{array}$ & $\stackrel{?}{\leftrightarrow}$ & $\begin{array}{l}\text { AGWC } \\
2016[26\end{array}$ \\
\hline
\end{tabular}

Table 1: The different "flavors" of well-composedness and their relationship on cubical grids.

topological boundary of the continuous analog of a set is a manifold is named well-composedness in the continuous sense or CWCness [29]. Well-composedness based on discrete surfaces in Alexandrov spaces is named wellcomposedness in the Alexandrov sense or AWCness [29]. The natural extension in $n$-D of well-composedness such that the connected components of a set or of the complementary of this set do not depend on the chosen connectivity is named well-composedness based on the equivalence of connectivities or EWCness [29]. Wellcomposedness based on critical configurations, i.e., such that a set is said well-composed iff it does not contain any critical configuration [29], is named digital wellcomposedness or DWCness [29]. Finally, well-composedness on arbitrary grids is renamed in this paper under the name of $\mathcal{A G}$-well-composedness.

On cubical grids, DWCness has been observed to be equivalent to CWCness and to AWCness in $2 \mathrm{D}$ and in $3 \mathrm{D}$; however, if these three flavours of well-composednesses are equivalent to EWCness in 2D, they are stronger than EWCness in 3D. Furthermore, DWCness, CWCness and AWCness have not been proved to be equivalent to each other in $n$-D, $n \geq 4$. Hence it is useful to see how these different flavors of well-composedness are related to each other (see Table 1). Observe that in $2 \mathrm{D}$ the $\mathrm{X}$-well-composednesses $(\mathrm{X}=\mathrm{A}, \mathrm{C}, \mathrm{D}$, $\mathrm{E})$ are equivalent, so a set/image is well-composed iff it is $\mathrm{X}$-well-composed $(\mathrm{X}=\mathrm{A}, \mathrm{C}, \mathrm{D}, \mathrm{E})$. On the other hand, in $3 \mathrm{D}$, the $\mathrm{X}$-well-composednesses $(\mathrm{X}=\mathrm{A}, \mathrm{C}$, D) are equivalent, so a set/image is well-composed iff it is $\mathrm{X}$-well-composed $(\mathrm{X}=\mathrm{A}, \mathrm{C}, \mathrm{D})$. In $n$-D, $n \geq 4$, the equivalences are not proved yet, so we need to specify the type of well-composedness we are dealing with (AWCness, CWCness, DWCness, or EWCness). Note that no link between $\mathcal{A G}$-well-composedness and the other flavours of well-composedness has been published yet in $n$-D.
Also, we will note that well-composed sets are counterparts of $n$-dimensional manifolds in the sense that they do not have singularities. This means that such sets can be directly used by algorithms that compute differential properties from digital data, bypassing the need for converting them to a continuous representation in order to compute the same properties; see [97, 99. for instance. Also, continuous representations obtained from well-composed images are consistent with their digital counterparts; having two consistent representations (digital and continuous) allows for algorithms to choose between the two in order to more effectively perform a certain task.

This state-of-art is organized as follow. Section 2 explains the origins of well-composedness. Section 3 recalls the definition of the generalization of digital wellcomposedness to $n$-D. Section 4 recalls the definitions of well-composednesses on complexes. Section 5 recalls the other flavours in matter of well-composedness. Section 6recalls how well-composedness has been extended from digital sets to digital gray-level images. Section 7 recalls the different methods to obtain well-composed sets or images. Section 8 and Section 9 recall the different topics and applications that are respectively related to well-composed sets and well-composed gray-level images. Finally, Section 10 concludes this paper.

\section{Where does well-composedness comes from?}

We recall in this section some fundamental notions of digital topology, some well-known topological paradoxes, and how these paradoxes led to the birth of a new class of images called well-composed sets.

\subsection{Digital topology in $\mathbb{Z}^{2}$}

Here are the basic definitions of digital topology 148 , 90. when we work in the digital plane $\mathbb{Z}^{2}$. Let $S$ be 


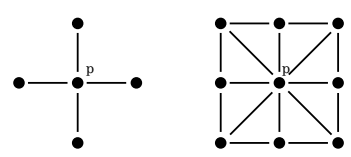

Fig. 1: Neighborhoods of a point $p \in \mathbb{Z}^{2}$.

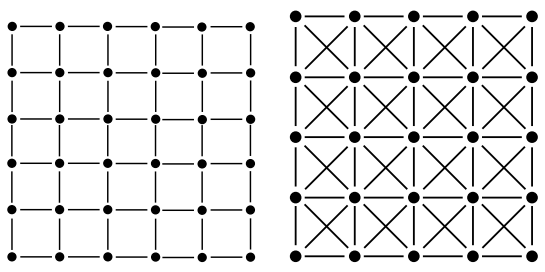

Fig. 2: Square grids using 4-adjacency on the left and 8 -adjacency on the right.

a subset of the digital plane, the points in $S$ will be termed foreground points, while those of its complement in the digital plane, $S^{c} \equiv \mathbb{Z}^{2} \backslash S$, will be termed the background points. Note that the background points (respectively, the foreground points) will be depicted using white or black points depending on the context. The 4-neighbors of a point $(x, y) \in \mathbb{Z}^{2}$ are the points $(x+1, y),(x-1, y),(x, y+1)$ and $(x, y-1)$. The 8 neighbors of a point $(x, y) \in \mathbb{Z}^{2}$ are its four 4-neighbors together with its four diagonal neighbors $(x+1, y+1)$, $(x+1, y-1),(x-1, y+1)$ and $(x-1, y-1)$. For $n \in$ $\{4,8\}$, the $n$-neighborhood of a point $P=(x, y) \in \mathbb{Z}^{2}$ is the set $\mathcal{N}_{n}(P)$ consisting of $P$ plus its $n$-neighbors. $\mathcal{N}_{n}^{*}(P)$ is the set of all $n$-neighbors of $P$ without $P$ itself: $\mathcal{N}_{n}^{*}(P)=\mathcal{N}_{n}(P) \backslash\{P\}$. Figure1 depicts on the left the 4-neighborhood and on the right the 8-neighborhood of a point $p \in \mathbb{Z}^{2}$.

Let $P, Q$ be two points of $\mathbb{Z}^{2}$. We say that a sequence of points $\left(P=P_{1}, \ldots, P_{n}=Q\right)$ of $\mathbb{Z}^{2}$ is a $n$ path, $n \in\{4,8\}$, from $P$ to $Q$ iff $P_{i} \in \mathcal{N}_{n}^{*}\left(P_{i-1}\right)$ for $i \in \llbracket 2, n \rrbracket$, and it is a path if it is a $n$-path for some $n \in\{4,8\}$. A set $X \subseteq \mathbb{Z}^{2}$ is said $n$-connected iff for every pair of points $P, Q \in X$, there exists a $n$-path in $X$ from $P$ to $Q$, and connected if it is connected for some $n \in\{4,8\}$. A $n$-component of a set $S \subseteq \mathbb{Z}^{2}$ is a maximal $n$-connected subset of $S$. Depending on whether 4- or 8-connectedness is used, we mean 4- or 8-components. A set $C \subset \mathbb{Z}^{2}$ is called a simple closed curve if it is connected and each of its points has exactly two neighbors in $C$. Depending on whether we use 4- or 8-neighborhoods, we call $C$ either a 4-curve or a 8-curve.
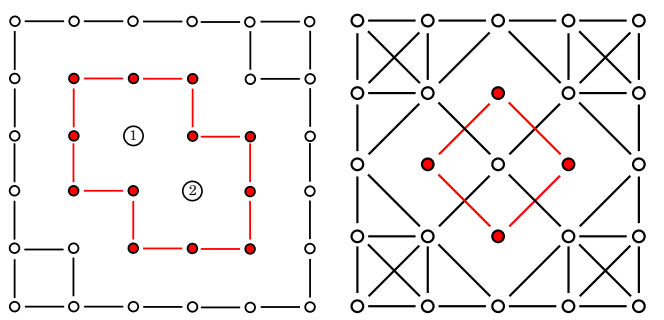

Fig. 3: Some connectivity paradoxes using 4-adjacency on the left and 8-adjacency on the right.
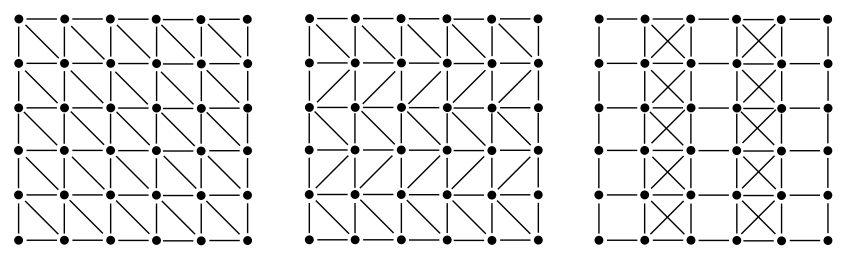

Fig. 4: Different square grids based on 6-adjacency.

\subsection{Connectivity paradoxes on cubical grids}

Let $V$ be the set $\mathbb{Z}^{2}$, and let $E \subset V \times V$ be the irreflexive symmetric binary relation such that any two points $p, q \in V$ verify $(p, q) \in E$ iff $p$ and $q$ are $n$ adjacent. We call the points of $V$ the vertices and the elements of $E$ the edges. We obtain this way a graph structure $\mathcal{G}=(V, E)$ based on the $n$-adjacency. These structures representing the digital plane supplied with the $n$-adjacency can be observed in Figure 2.

Now, assuming that we have a set of foreground points $S \subset \mathbb{Z}^{2}$ that is given and which depicts a 4 - or a 8-curve in $\mathbb{Z}^{2}$, we could hope that the Jordan Separation Theorem which states that a simple closed curve separates $\mathbb{R}^{2}$ into two components, the interior which is bounded and the exterior which is not bounded, holds as in the continuous world. However, when we draw a 4-curve in the digital plane supplied with the 4-adjacency as shown on the left of Figure 3 this curve separates the digital plane into 3 components, two of them are bounded and the third is unbounded. In a certain manner, we have two "interiors". The Jordan Separation Theorem does not hold in discrete spaces using 4-adjacency. We can also draw an 8-curve in the digital plane, as shown on the right of Figure 3, and we obtain that the complement of the 8-curve is an only connected component. The "interior" and the "exterior" are the same component. Then the Jordan Separation Theorem fails with 8-adjacency too.

Rosenfeld called these phenomena the connectivity paradoxes [153, 90,104 and explained that this failure follows from the fact that we use the same adjacency for the foreground and the background. Effectively, we can 

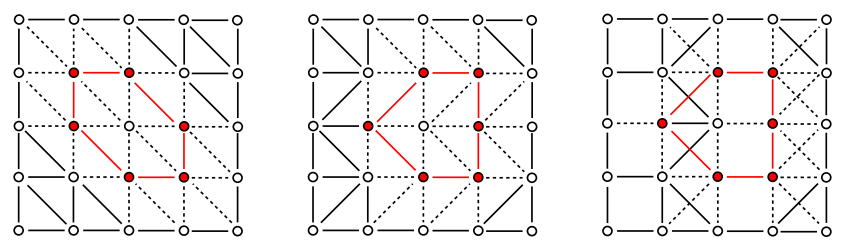

Fig. 5: A 6-curve does not always separates the digital plane even if we use 6-adjacency.
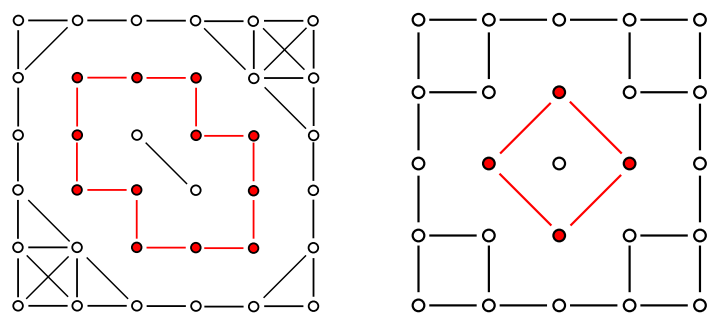

Fig. 6: (4,8)-adjacency on the left and $(8,4)$-adjacency on the right.

remark that when we use 6-adjacency, such as depicted in Figure 4 a 6-curve does not always satisfy the Jordan Separation Theorem (see Figure 5): it works using the first or second grids but not the other.

Note that digital subsets of $\mathbb{Z}^{2}$ are in bijection with digital binary images: a 2D digital binary image [102 (p. 102) is a 4 -uple denoted by $\left(\mathbb{Z}^{2}, X, k, l\right)$ where $X$ is a subset of $\mathbb{Z}^{2}$ such that either $X$ or $X^{c}$ is finite; $X$ corresponds to the foreground and is associated with the $k$-adjacency, and $X^{c}:=\mathbb{Z}^{2} \backslash X$ corresponds to the background of the image and is associated with the $l$ adjacency. Equivalently, this image can be interpreted as the characteristic function of the set $X$ in $\mathbb{Z}^{2}$, that is, a mapping $I$ from $\mathbb{Z}^{2}$ to $\{0,1\}$ such that $I(p)=1$ if $p \in X$ and $I(p)=0$ if $p \in X^{c}$. This way, these same connectivity paradoxes can happen on binary images.

\subsection{Using dual pairs of connectivities}

Using a dual pair of adjacencies, as recommended in 50] for the first time, can be helpful. The $(8,4)$-adjacency, meaning that we use 8-adjacency for the foreground and 4 -adjacency for the background, or the $(4,8)$-adjacency, meaning that we use 4-adjacency for the foreground and 8-adjacency for the background, make the Jordan Separation Theorem (JST) true at the condition that an 8-curve (respectively, a 4-curve) is made by at least 4 points (respectively, by at least 8 points) to avoid pathological cases. This is depicted in Figure 6 on the left, the 4-curve separates the plane into two 8-components, and on the right, the 8 -curve separates the plane into two 4-components.

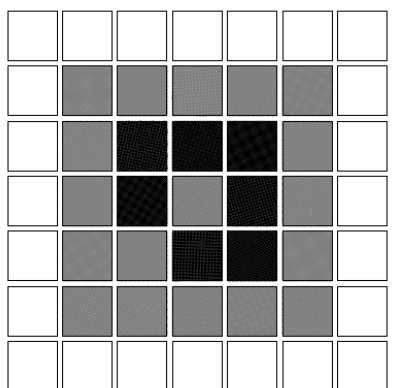

Fig. 7: The gray set is made of one or two components depending on the associated connectivity 118 .

Using a dual pair of connectivities is efficient but has a main drawback: the result of an algorithm often depends on the chosen pair. In other words, we have to choose, depending on the application, one particular pair and we are not always able to know a priori which one is the best to our needs. Indeed, the set of connected components of a given set clearly depends on the chosen pair, and then the consequences can be unexpected or unstable results in some applications like object counting [90].

Using digital multi-label images, that is, digital images with possibly more than two "colors", we may consider each label as the foreground with one connectivity, and the union of the other labels as the background with a dual connectivity. However, this approach can be problematic: some label can be associated with different connectivities if it is considered as the foreground or part of the background, leading to ambiguities. For example, it can be made of one component at a given time and of two components at another time [118] (see Figure 7). Furthermore, in this way we consider only each label relatively to its associated background; the topological relationship between the different labels is then lost [142,11,111. Another approach is to consider that the multi-label image can be represented with a connected components tree 114 where the root is the infinite background, and the rest of the tree represent the nested relationship between the connected components into the image. This way, the new representation of the image is "isomorphic" to a binary image; this approach has then been called binary modeling [114,122]. However, this last method assumes that the topological structure of the connected components of the labels has no ambiguity, and then can only be applied on "simple images" [118.

An alternative to these methods is to work directly with images where we can associate the same connectivity to the foreground and to the background without 


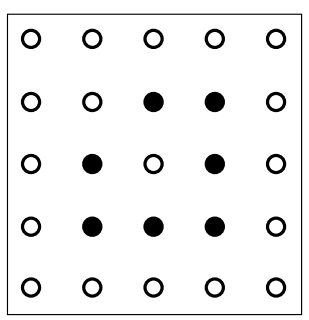

Fig. 8: A set which is weakly well-composed but not well-composed [104].

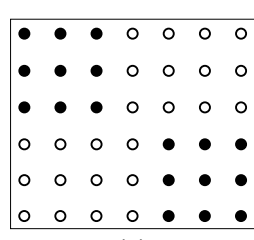

(a)

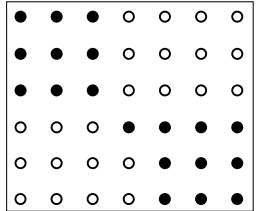

(b)

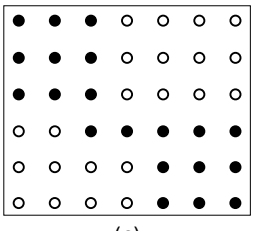

(c)
Fig. 9: The (black) sets are well-composed in $(a)$ and $(c)$, but the (black) set in $(b)$ is neither well-composed nor weakly well-composed [104].

having any topological paradox, ambiguity, or collision between sets, they are called well-composed images. The seminal definition of this class of images, much easier to manage in practice, is developed below.

\subsection{The seminal definition of well-composedness}

In 1995, Latecki et al. introduced what they called wea$k l y$ well-composed sets [104, that is, subsets of $\mathbb{Z}^{2}$ such that any of their 8-components are also 4-components. For example, as shown in Figure 8 [104, this set is weakly well-composed, since it is made of one 8-component (in black) which is also a 4-component.

Since this definition is not self-dual, that is, $S$ weakly well-composed does not imply that its complementary is well-composed, Latecki strenghtened this definition in the following manner: a subset $S$ of $\mathbb{Z}^{2}$ is said wellcomposed 104 iff $S$ and its complement $S^{c}$ in $\mathbb{Z}^{2}$ are both weakly well-composed. As shown in Figure 9 , the (black) set $S$ in Figure 9(a) is made of two 8-components which are also 4-components. The set in Figure $9(c)$ is made of only one 8-component, which is also a 4component. On the contrary, the set in Figure $9(b)$ is made of only one 8-component, which is made of two 4components, and then is neither weakly well-composed nor well-composed.

Then Latecki reformulated the notion of well-composedness using local 4-connectivity 104] a set $S \subseteq \mathbb{Z}^{2}$ is said locally 4-connected iff the points of $S$ in the 8neighborhood of any point of $S$ are 4-connected, i.e.,

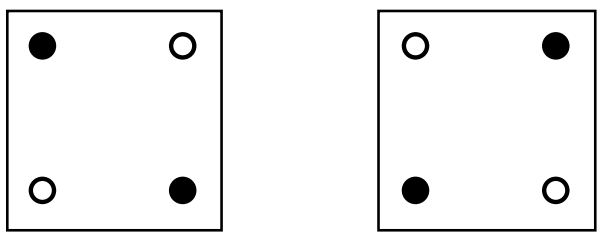

Fig. 10: Forbidden patterns into well-composed sets [104].

$S \cap \mathcal{N}_{8}(P)$ is 4-connected for every point $P$ in $S$. Notice that this notion is self-dual: $S$ is locally 4 -connected iff $S^{c}$ is locally 4-connected. Latecki proposed then the following theorem [104]: any set $S \subseteq \mathbb{Z}^{2}$ is well-composed iff it is locally 4-connected. This way, it was clear that the patterns depicted in Figure 10, that they called "critical configurations", and representing two points which are 8-adjacent but not 4-adjacent, cannot occur in a well-composed set.

In 2015, Boutry et al. 29] have extended the seminal definition of well-composedness to $n$-D, $n \geq 2$, in such a manner that a digital set $X \subset \mathbb{Z}^{n}$ is said well-composed based on the equivalence of connectivities (EWC) iff the set of $\left(3^{n}-1\right)$-connected components $X$ (respectively, of $\left.X^{c}\right)$ is equal to the set of $2 n$-connected components of $X$ (respectively, of $X^{c}$ ). This definition will be detailed in Section 5

\section{Digital well-composedness in $n$-D}

In this section, we recall the basics about digital topology extended to $n$-D, $n \geq 2$, and then we recall the definition and the characterization proposed in [29] of digital well-composedness.

\subsection{Basics of digital topology in $\mathbb{Z}^{n}$}

Let $n \geq 2$ be a (finite) integer called the dimension. Now, let $\mathbb{B}=\left\{e^{1}, \ldots, e^{n}\right\}$ be the (orthonormal) canonical basis of $\mathbb{Z}^{n}$. We use the notation $x_{i}$, where $i$ belongs to $\llbracket 1, n \rrbracket$, to determine the $i^{\text {th }}$ coordinate of the vector $x \in \mathbb{Z}^{n}$. We recall that the $L^{1}$-norm of a point $x \in \mathbb{Z}^{n}$ is denoted by $\|\cdot\|_{1}$ and is equal to $\sum_{i \in \llbracket 1, n \rrbracket}\left|x_{i}\right|$ where |.| is the absolute value. Also, the $L^{\infty}$-norm is denoted by $\|\cdot\|_{\infty}$ and is equal to $\max _{i \in \llbracket 1, n \rrbracket}\left|x_{i}\right|$.

For a given point $x \in \mathbb{Z}^{n}$, the $2 n$-neighborhood in $\mathbb{Z}^{n}$ is noted $\mathcal{N}_{2 n}(x)$ and is equal to $\left\{y \in \mathbb{Z}^{n} ;\|x-y\|_{1} \leq 1\right\}$. In other words,

$$
\mathcal{N}_{2 n}(x)=\{x\} \cup\left\{x-e^{1}, x+e^{1}, \ldots, x-e^{n}, x+e^{n}\right\} .
$$



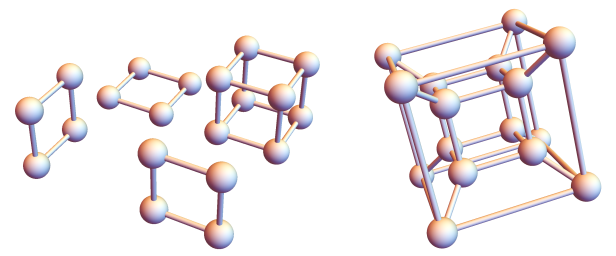

Fig. 11: 2D, 3D and 4D blocks.

For a given point $x \in \mathbb{Z}^{n}$, the $\left(3^{n}-1\right)$-neighborhood in $\mathbb{Z}^{n}$ is noted $\mathcal{N}_{3^{n}-1}(x)$ and is equal to $\left\{y \in \mathbb{Z}^{n} ; \| x-\right.$ $\left.y \|_{\infty} \leq 1\right\}$. In other words, $\mathcal{N}_{3^{n}-1}(x)$ equals:

$$
\left\{x+\sum_{i \in \llbracket 1, n \rrbracket} \lambda_{i} e^{i} ; \lambda_{i} \in\{-1,0,1\}, \forall i \in \llbracket 1, n \rrbracket\right\} .
$$

From now on, let $\xi$ be a value in $\left\{2 n, 3^{n}-1\right\}$.

The starred $\xi$-neighborhood of $x \in \mathbb{Z}^{n}$ is noted $\mathcal{N}_{\xi}^{*}(x)$ and is equal to $\mathcal{N}_{\xi}(x) \backslash\{x\}$. An element of the starred $\xi$-neighborhood of $x \in \mathbb{Z}^{n}$ is called a $\xi$-neighbor of $x$ in $\mathbb{Z}^{n}$. Two points $x, y \in \mathbb{Z}^{n}$ such that $x \in \mathcal{N}_{\xi}^{*}(y)$ or equivalently $y \in \mathcal{N}_{\xi}^{*}(x)$ are said to be $\xi$-adjacent.

Let $x, y$ be two points in $\mathbb{Z}^{n}$ and $X$ be a subset of $\mathbb{Z}^{n}$. A finite sequence $\left(p^{0}, \ldots, p^{k}\right)$ is a $\xi$-path if and only if $p^{0}$ is $\xi$-adjacent only to $p^{1}, p^{k}$ is $\xi$-adjacent only to $p^{k-1}$, and if for $i \in \llbracket 1, k-1 \rrbracket, p^{i}$ is $\xi$-adjacent only to $p^{i-1}$ and to $p^{i+1}$. Such paths are said to be of length $k$.

A subset $X$ of $\mathbb{Z}^{n}$ such that $\operatorname{Card}(X)$ or $\operatorname{Card}\left(\mathbb{Z}^{n} \backslash\right.$ $X)$ is finite is said to be a digital set. A digital set $X \subset$ $\mathbb{Z}^{n}$ is said $\xi$-connected iff for any pair of points $x, y \in X$, there exists a $\xi$-path joining them into $X$. A subset $C$ of $X$ which is $\xi$-connected and which is maximal in the inclusion sense, that is, there is no subset of $X$ which is greater than $C$ and which is $\xi$-connected, is said to be a $\xi$-component of $X$.

A point $x \in \mathbb{Z}^{n}$ is said to be $\xi$-connected to a set $Y \subseteq \mathbb{Z}^{n}$ iff there exists a point $y \in Y$ such that $x$ and $y$ are $\xi$-neighbors or equal. Two sets $X, Y \subseteq \mathbb{Z}^{n}$ are said to be $\xi$-connected iff there exists $x \in X$ such that $x$ and $Y$ are $\xi$-connected.

\subsection{Definition of DWCness in $n$-D}

To be able to recall the definition of digital well-composedness, named this way due to the "digital patterns" on which it is based, we first have to recall the elementary notions of block, antagonism, and critical configurations in $\mathbb{Z}^{n}$.

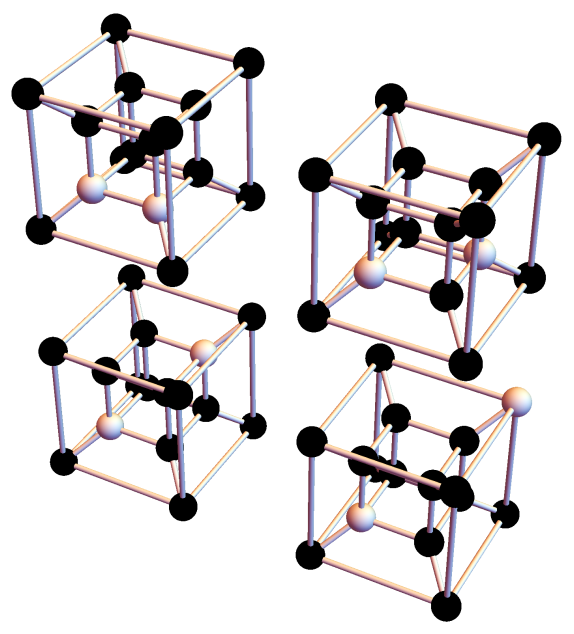

Fig. 12: In the raster scan order: in this $4 \mathrm{D}$ example, the white points are 1-antagonists, 2-antagonists, 3 -antagonists, and 4-antagonists.

Given a point $z \in \mathbb{Z}^{n}$ and a family of vectors $\mathcal{F}=$ $\left(f^{1}, \ldots, f^{k}\right) \subseteq \mathbb{B}$, we define the block associated with the pair $(z, \mathcal{F})$ in this way:

$S(z, \mathcal{F})=\left\{z+\sum_{i \in \llbracket 1, k \rrbracket} \lambda_{i} f^{i} \mid \lambda_{i} \in\{0,1\}, \forall i \in \llbracket 1, k \rrbracket\right\}$.

A subset $S \subset \mathbb{Z}^{n}$ is called a block iff there exists a pair $(z, \mathcal{F}) \in \mathbb{Z}^{n} \times \mathcal{P}(\mathbb{B})$ such that $S=S(z, \mathcal{F})$. Note that a block which is associated with a family $\mathcal{F} \in \mathcal{P}(\mathbb{B})$ of cardinality $k \in \llbracket 0, n \rrbracket$ is said to be of dimension $k$, what will be denoted by $\operatorname{dim}(S)=k$. Figure 11 shows 2D, 3D and 4D blocks. We will denote the set of blocks of $\mathbb{Z}^{n}$ by $\mathcal{B}\left(\mathbb{Z}^{n}\right)$.

Using this notion of blocks, we can define antagonism. Two points $p, q$ belonging to a block $S \in \mathcal{B}\left(\mathbb{Z}^{n}\right)$ are said to be antagonists in $S$ iff their distance equals the maximal distance using the $L^{1}$-norm between two points into $S$. In other words, two points $p$ and $q$ in $\mathbb{Z}^{n}$ are antagonists in $S \in \mathcal{B}\left(\mathbb{Z}^{n}\right)$ iff $p, q \in S$ such that:

$$
\|p-q\|_{1}=\max \left\{\|x-y\|_{1} ; x, y \in S\right\},
$$

and in this case we write that $q=\operatorname{antag}_{S}(p)$ or equivalently $p=\operatorname{antag}_{S}(q)$. The antagonist of a point $p$ in a block $S \in \mathcal{B}\left(\mathbb{Z}^{n}\right)$ containing $p$ exists and is unique. Sometimes we will use the notation $S(p, q)$ where $p, q \in$ $\mathbb{Z}^{n}$ are $\left(3^{n}-1\right)$-neighbors to indicate the block in $\mathcal{B}\left(\mathbb{Z}^{n}\right)$ such that $p$ and $q$ are antagonists in this block.

Also, two points which are antagonists in a block of dimension $k \in \llbracket 0, n \rrbracket$ are said $k$-antagonists. In this case, $k$ of their coordinates differ, and they differ from a 


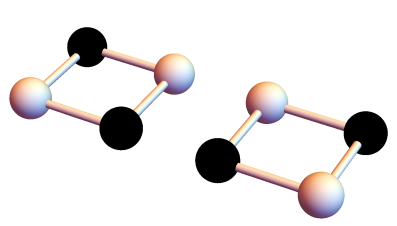

Fig. 13: The white points on the left draw a $2 \mathrm{D}$ primary critical configuration, and the white points on the right draw a secondary 2D critical configuration.

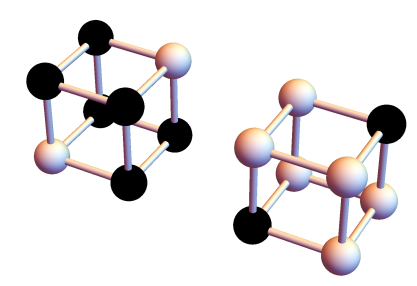

Fig. 14: The white points on the left draw a 3D primary critical configuration, and the white points on the right draw a secondary 3D critical configuration.

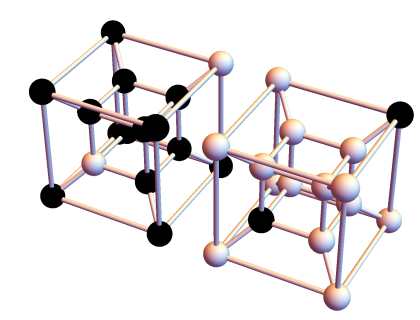

Fig. 15: The white points on the left draw a 4D primary critical configuration, and the white points on the right draw a secondary 4D critical configuration.

value 1 , the other coordinates being equal. Two points which are 0 -antagonists are equal, two points which are 1 -antagonists in a block of $\mathbb{Z}^{n}$ are $2 n$-neighbors in $\mathbb{Z}^{n}$, and two points which are $n$-antagonists in a block of $\mathbb{Z}^{n}$ are $\left(3^{n}-1\right)$-neighbors in $\mathbb{Z}^{n}$. See Figure 12 for different possible pair of antagonists (in white) in a $4 \mathrm{D}$ space.

Now, we are able to define critical configurations of dimension $k \in \llbracket 2, n \rrbracket$ in a $n$-D space: let $X \subset \mathbb{Z}^{n}$ be a digital set, and let $S \in \mathcal{B}\left(\mathbb{Z}^{n}\right)$ be a block of dimension $k \in \llbracket 2, n \rrbracket$. We say that $X$ contains a primary critical configuration of dimension $k$ in the block $S$ iff $X \cap S=\left\{p, p^{\prime}\right\}$ with $p, p^{\prime} \in S$ two points that are antagonists into $S$. We say that $X$ contains a secondary critical configuration of dimension $k$ in the block $S$ iff $X \cap S=S \backslash\left\{p, p^{\prime}\right\}$ with $p, p^{\prime} \in S$ two points that are antagonists into $S$. More generally, a critical configuration of dimension $k \in \llbracket 2, n \rrbracket$ is either a primary or a secondary critical configuration of dimension $k$.
Figures 13, 14 and 15 depict 2D, 3D, and 4D critical configurations.

There comes the definition of digitally well-composed sets in $\mathbb{Z}^{n}$ : a digital set $X \subset \mathbb{Z}^{n}$ is said digitally well-composed or $D W C$ iff it does not contain any critical configurations, that is, for any block $S \in \mathcal{B}\left(\mathbb{Z}^{n}\right)$, the restriction $X \cap S$ is neither a primary nor a secondary critical configuration. Obviously, this definition is self-dual, since a set $X \subset \mathbb{Z}^{n}$ contains a primary (respectively, a secondary) critical configuration in the block $S \in \mathcal{B}\left(\mathbb{Z}^{n}\right)$ iff its complement $X^{c}$ contains a secondary (respectively, a primary) critical configuration in this same block $S$.

Note that this definition is based on local patterns, by contrast to EWCness (defined later in Section 5 ) which is based on connected components, and then is global.

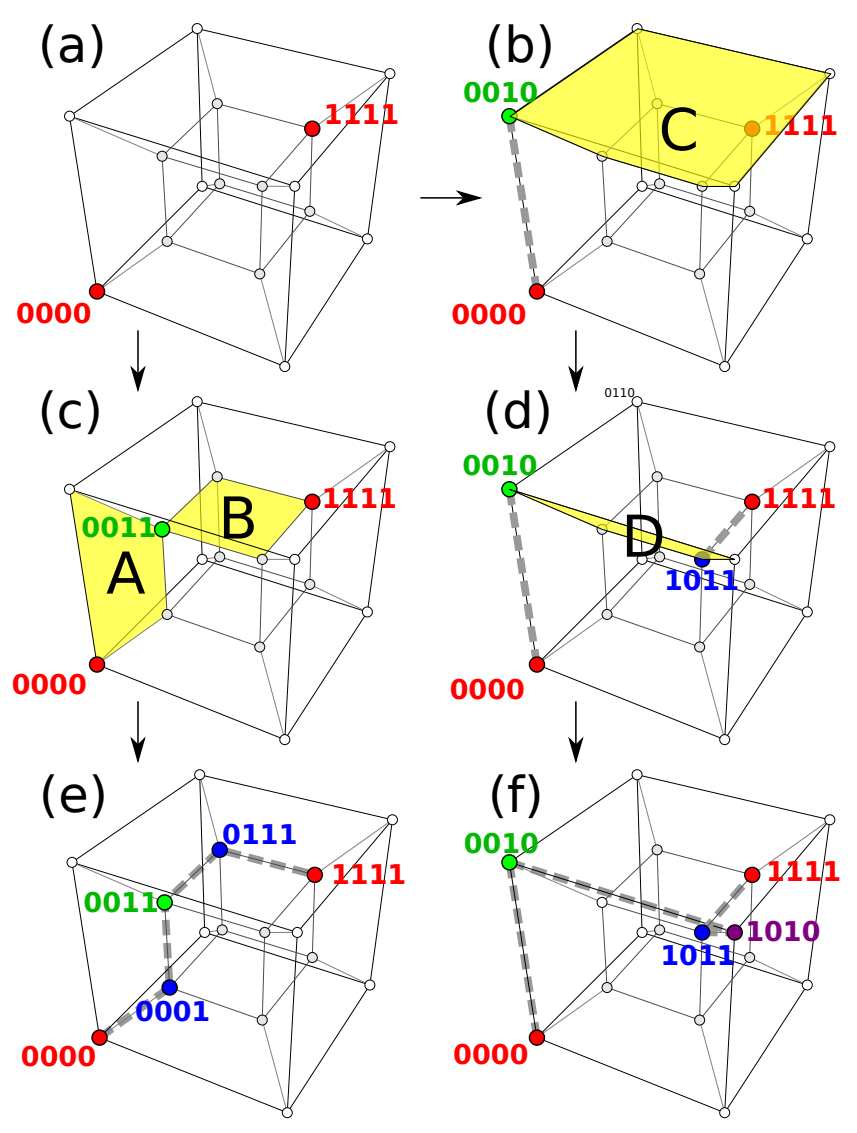

Fig. 16: Step-by-step construction of the $2 n$-path joining the two (red) antagonists into $X \cap S$ into $\mathbb{Z}^{n}$.

We can reformulate digital well-composedness based on $2 n$-paths [29]: a set $X \subset \mathbb{Z}^{n}$ is digitally well-composed iff, for any block $S \in \mathcal{B}\left(\mathbb{Z}^{n}\right)$ and for any pair of points $\left(p, \operatorname{antag}_{S}(p)\right)$ such that they belong to $X \cap S$ 
(resp. $S \backslash X), p$ and $\operatorname{antag}_{S}(p)$ are $2 n$-connected in $X \cap S$ (resp. in $S \backslash X$ ).

This reasoning is illustrated in Figure 16 two antagonists, depicted in red in the block $S$ (the tesseract), are assumed to belong to a digitally well-composed set $X \subset \mathbb{Z}^{n}$, which is shown in Figure 16(a). Since the two red points $(0,0,0,0)$ and $(1,1,1,1)$ belong to $X$ and are 4-antagonists in $S$, there exists at least one more point in the block $S$ belonging to $X$ (in the contrary case, $X$ contains a critical configuration, which is impossible by hypothesis). A first possibility is shown in Figure 16(b), and a second possibility is shown in Figure 16(c), where the green point depicts this additional point. Let us treat first the case corresponding to Figure 16(b): since the points $(0,0,1,0)$ and $(1,1,1,1)$ are 3 -antagonists in the $3 \mathrm{D}$ block $C$ depicted in yellow, there must be at least one more point in this block which belongs to $X$ (for the same reason as before), and then we obtain that the blue point $(1,0,1,1)$ belongs to $X$, which is shown in Figure 16(d). Applying recursively the reasoning until $X$ does not contain any critical configuration, we obtain that the point $(1,0,1,0)$ also belongs to $X$, which is shown in purple in Figure $16(f)$. Finally, we obtain a $2 n$-path joining the two red points $(0,0,0,0)$ to $(1,1,1,1)$ into $X \cap S$. Let us now treat the case corresponding to Figure $16(c)$ : if $(0,0,0,0)$ and $(0,0,1,1)$, which are 2-antagonists, are the only points of $X$ in the block $A, X \cap A$ is a critical configuration, then there exists an additional point among $(0,0,1,0)$ and $(0,0,0,1)$ which belongs to $X$. The same happens in the block $B$ where at least $(0,0,1,1)$ and $(1,1,1,1)$ belongs to $X$ : at least $(0,1,1,1)$ or $(1,0,1,1)$ must belong to $X$. Let us assume that $(0,0,0,1)$ and $(0,1,1,1)$ belong to $X$, we obtain Figure $16(e)$ where a $2 n$-path joins the two red points $(0,0,0,0)$ to $(1,1,1,1)$ in $X \cap S$. Obviously, the reasoning is similar when $(0,0,0,0)$ and $(1,1,1,1)$ belong to $X^{c}$. In this case, we obtain that a $2 n$-path joins these two points in $X^{c} \cap S$, thanks to self-duality of digital well-composedness.

\section{Well-composedness on complexes}

After having recalled some basics in matter of discrete topology related to Alexandrov spaces, we recall the definition of well-composedness in the Alexandrov sense or $A W C n e s s$ introduced in [127] by Najman and Géraud. Then, we present the most used immersions from $\mathbb{Z}^{n}$ into Khalimsky grids, denoted by $\mathbb{H}^{n}$, and we will see the link between DWCness and AWCness into these cubical grids. Finally, we will recall the definition of wellcomposed polytopal complexes according to Stelldinger.

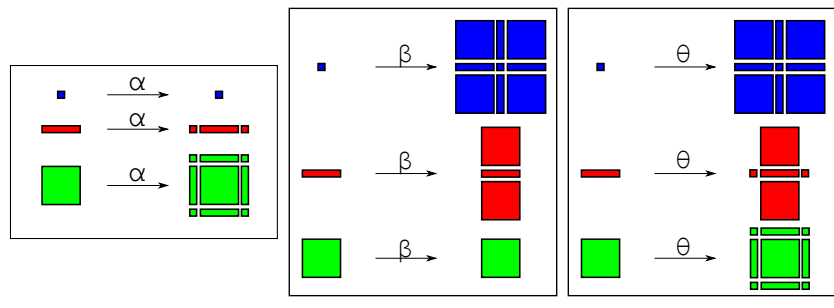

Fig. 17: Basic operators in axiomatic digital topology: $\alpha$ is the combinatorial closure, $\beta$ is the combinatorial opening, and $\theta$ is the neighborhood.

\subsection{WCness on Alexandrov spaces}

Let $X$ be any set, and let $\mathcal{U}$ be a set of subsets of $X$ s.t. $X, \emptyset \in \mathcal{U}$, any union of any family of elements in $\mathcal{U}$ belongs to $\mathcal{U}$, and any finite intersection of any family of elements in $\mathcal{U}$ belongs to $\mathcal{U}$. Then $\mathcal{U}$ is a topology 85 2], and the pair $(X, \mathcal{U})$ is called a topological space. We abusively say that $X$ is a topological space, assuming it is supplied with its topology $\mathcal{U}$. The elements of $\mathcal{U}$ are called the open sets of $(X, \mathcal{U})$, and the complement of an open set is said to be a closed set [2]. A set $N$ containing an element $p$ of a topological space $X$ s.t. there exists $U \in \mathcal{U}$ s.t. $p \in U \subseteq N$ is said to be a neighborhood of $p$ into $X$. We say that a topological space $(X, \mathcal{U})$ verifies the $T_{0}$ axiom of separation $[4,85,2$ iff for any two different elements $X$, at least one of them admits an open neighborhood not containing the other element. A topological space which verifies the $T_{0}$ axiom of separation is said to be a $T_{0}$-space, a topological space $X$ is said discrete [6] iff the intersection of any family of open sets of $X$ is open in $X$, and a discrete $T_{0}$-space is said to be an Alexandrov space [52].

Let $\Lambda$ be an arbitrary set. A binary relation [15] $R$ on $\Lambda$ is a subset of $\Lambda \times \Lambda$, and for any $x, y \in \Lambda$, we denote by $x R y$ the fact that $(x, y) \in R$, or equivalently $x \in R(y)$. A binary relation $R$ is said reflexive iff, $\forall x \in \Lambda, x R x$, asymmetric iff, $\forall x, y \in \Lambda$, $x R y$ and $y R x$ implies $x=y$, and transitive iff, $\forall x, y, z \in \Lambda, x R y$ and $y R z$ implies $x R z$. Also, we denote by $R^{\square}$ the binary relation defined such that, $\forall x, y \in \Lambda,\left\{x R^{\square} y\right\} \Leftrightarrow\{x R y$ and $x \neq y\}$. An order relation [15] on $\Lambda$ is a binary relation $R$ s.t. it is reflexive, asymmetric, and transitive, and a set $\Lambda$ of arbitrary elements supplied with an order relation $R$ on $X$ is denoted $(\Lambda, R)$ or $|\Lambda|$ and is called a poset $[15] ; \Lambda$ is called the domain of $|\Lambda|$.

According to Alexandrov (Th. 6.52, p. 28 of [2]), we can identify any poset $|X|=(X, R)$ with the Alexandrov space induced by the order relation $R$, that is, by considering that a subset $A$ of $X$ is closed iff for any $x, y \in X, x \in A \wedge y R x \Rightarrow y \in A$. This way, we 
consider equivalently $|X|=\left(X, \alpha_{X}\right)$ as a poset and as an Alexandrov space induced by the order relation $\alpha_{X}$. So, let $\left(X, \alpha_{X}\right)$ be a poset and $p$ an element of $X$, we define the combinatorial closure $\alpha_{X}(p)$ of $p$ in $|X|$ as the set $\left\{q \in X ;(q, p) \in \alpha_{X}\right\}$, the combinatorial opening $\beta_{X}(p)$ of $p$ in $|X|$ as the set $\left\{q \in X ;(p, q) \in \alpha_{X}\right\}$, and $\theta_{X}(p)=\alpha_{X}(p) \cup \beta_{X}(p) ; \alpha(p)$ is then the smallest closed set containing $\{p\}$ and $\beta_{X}(p)$ is the smallest open set containing $\{p\}$ in $X$. Some examples of the operators $\alpha, \beta$, and $\theta$ are depicted in Figure 17. Also, we define, $\forall S \subseteq X, \alpha_{X}(S)=\cup_{p \in S} \alpha_{X}(p), \beta_{X}(S)=\cup_{p \in S} \beta_{X}(p)$, and $\theta_{X}(S)=\cup_{p \in S} \theta_{X}(p)$.

Now, let $S$ be a subset of $X$. The suborder [15] of $|X|$ relative to $S$ is the poset $|S|=\left(S, \alpha_{S}\right)$ s.t. $\alpha_{S}=$ $\alpha_{X} \cap S \times S$; we have then, for any $x \in S, \alpha_{S}(x) \equiv$ $\alpha_{X}(x) \cap S, \beta_{S}(x) \equiv \beta_{X}(x) \cap S$, and $\theta_{S}(x) \equiv \theta_{X}(x) \cap S$. For any suborder $|S|$ of $|X|$, we denote by $\operatorname{Int}_{X}(S)$ the open set $\left\{h \in X ; \beta_{X}(h) \subseteq S\right\}$. A set $S \subseteq X$ is said to be a regular open set (resp. a regular closed set) iff $S=\operatorname{Int}_{X}\left(\alpha_{X}(S)\right)\left(\right.$ resp. $\left.S=\alpha_{X}\left(\operatorname{Int}_{X}(S)\right)\right)$.

We call relative topology [52] induced in $S$ by $\mathcal{U}$ the set of all the sets which can be written $U \cap S$ where $U \in \mathcal{U}$. A set which is open in the relative topology of $S$ is said to be a relatively open set [52]. A set $S \subseteq X$ is then said to be connected iff there is no decomposition $S=T_{1} \cup T_{2}$ such that $T_{1} \cap T_{2}=\emptyset$, both $T_{1}, T_{2} \neq \emptyset$, and relatively open sets with respect to $S$. The largest connected set in $(X, \mathcal{U})$ containing $p \in X$ is called the component [2] of the point $p$ in $(X, \mathcal{U})$ and we denote it $\mathcal{C C}(X, p)$. When $(X, \mathcal{U})$ is non-empty, the set of maximal components of $X$ in the inclusion sense is denoted by $\mathcal{C C}(X)$ and is called the set of connected components of $X$. We call path [15] into $S \subseteq X$ a finite sequence $\left(p^{0}, \ldots, p^{k}\right)$ such that for all $i \in \llbracket 1, k \rrbracket, p^{i} \in \theta_{X}^{\square}\left(p^{i-1}\right)$, and we say that a set $S \subseteq X$ is path-connected [15] iff for any points $p, q$ in $S$, there exists a path into $S$ joining them. Since $|X|$ is an Alexandrov space, any subset $S$ of $X$ is connected iff it is path-connected [52,15].

The $\operatorname{rank} \rho(x,|X|)$ of an element $x$ in $|X|$ is 0 if $\alpha_{X}^{\square}(x)=\emptyset$ and is equal to $\max _{y \in \alpha_{X}^{\square}(x)}(\rho(y,|X|))+1$ otherwise. The rank of a poset $|X|$ is denoted by $\rho(|X|)$ and is equal to the maximal rank of its elements. An element $x$ of $X$ such that $\rho(x,|X|)=k$ is called $k$ face [15] of $X$.

Let $|X|=\left(X, \alpha_{X}\right)$ be a poset. $|X|$ is said countable iff its domain $X$ is countable. Also, $|X|$ is said locally finite iff for any element $x \in X$, the set $\theta_{X}(x)$ is finite. A poset which is countable and locally finite is said to be a $C F$-order [15. Now let us recall the definition of (discrete) $n$-surfaces [55]. Let $|X|=\left(X, \alpha_{X}\right)$ be a CForder, the poset $|X|$ is said to be either a $(-1)$-surface iff $X=\emptyset$, or a 0 -surface iff $X$ is made of two different

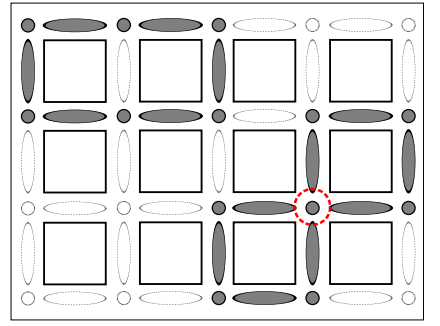

Fig. 18: On the left, a discrete 1-surface is a simple closed curve, and on the right a poset which is not a discrete 1-surface since it contains a pinch circled in red.

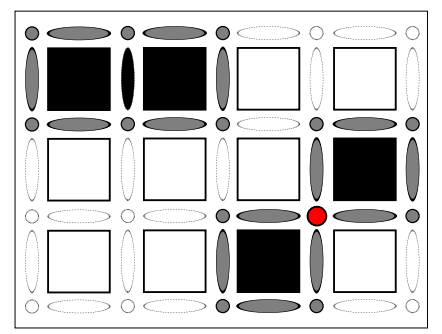

Fig. 19: An example of AWC set on the left, and an example of a set which is not AWC on the right.

elements $x, y \in X$ such that $x \notin \theta_{X}^{\square}(y)$, or an $n$-surface, $n \geq 1$, iff $|X|$ is connected and for any $x \in X,\left|\theta_{X}^{\square}(x)\right|$ is a $(n-1)$-surface. Figure 18 shows an example of a poset which is a 1-surface and an example of poset which is not a 1-surface (see the "pinch" circled in red).

Then, the boundary [127] of a digital subset $S$ in an Alexandrov space $X$ of rank $n \geq 1$ is the set:

$$
\alpha_{X}(S) \cap \alpha_{X}(X \backslash S),
$$

and $S$ is said well-composed in the sense of Alexandrov $(A W C)$ iff the connected components of its boundary are discrete $(n-1)$-surfaces. Examples of AWC and not AWC sets are depicted in Figure 19

\subsection{Khalimsky grids}

The Khalimsky grid $\left[88\right.$ of dimension $n$ is denoted $\left|\mathbb{H}^{n}\right| \equiv$ $\left(\mathbb{H}^{n}, \subseteq\right)$ and is defined as the poset such that $\mathbb{H}_{0}^{1}=$ $\{\{a\} ; a \in \mathbb{Z}\}, \mathbb{H}_{1}^{1}=\{\{a, a+1\} ; a \in \mathbb{Z}\}, \mathbb{H}^{1}=\mathbb{H}_{0}^{1} \cup$ $\mathbb{H}_{1}^{1}$, and $\mathbb{H}^{n}=\left\{h_{1} \times \cdots \times h_{n} ; \forall i \in \llbracket 1, n \rrbracket, h_{i} \in \mathbb{H}^{1}\right\}$. For any $h \in \mathbb{H}^{n}$, we have the following equalities: $\alpha(h) \equiv$ $\alpha_{\mathbb{H}^{n}}(h)=\left\{h^{\prime} \in \mathbb{H}^{n} ; h^{\prime} \subseteq h\right\}, \beta(h) \equiv \beta_{\mathbb{H}^{n}}(h)=\left\{h^{\prime} \in\right.$ $\left.\mathbb{H}^{n} ; h \subseteq h^{\prime}\right\}$, and $\theta(h) \equiv \theta_{\mathbb{H}^{n}}(h)=\left\{h^{\prime} \in \mathbb{H}^{n} ; h^{\prime} \subseteq\right.$ $h$ or $\left.h \subseteq h^{\prime}\right\}$. For any suborder $|X|$ of $\left|\mathbb{H}^{n}\right|$, we obtain that $\alpha_{X}(h)=\left\{h^{\prime} \in X ; h^{\prime} \subseteq h\right\}, \beta_{X}(h)=\left\{h^{\prime} \in\right.$ $\left.X ; h \subseteq h^{\prime}\right\}$, and $\theta_{X}(h)=\left\{h^{\prime} \in X ; h^{\prime} \subseteq h\right.$ or $\left.h \subseteq h^{\prime}\right\}$. Any element $h$ of $\mathbb{H}^{n}$ which is the Cartesian product of 
$k$ elements, with $k \in \llbracket 0, n \rrbracket$, of $\mathbb{H}_{1}^{1}$ and of $(n-k)$ elements of $\mathbb{H}_{0}^{1}$ is called a $k$-face of $\mathbb{H}^{n}$ and is said to be of dimension $k$ [95], which is denoted by $\operatorname{dim}(h)=k$, and the set of all the $k$-faces of $\mathbb{H}^{n}$ is denoted by $\mathbb{H}_{k}^{n}$. In the Khalimsky grids, the dimension is equal to the rank. Furthermore, for any $n \geq 1,\left|\mathbb{H}^{n}\right|$ is an Alexandrov space [15]. Finally, let $A, B$ be two subsets of $\mathbb{H}^{n}$, we say that $A$ and $B$ are separated iff $(A \cap(\beta(B)) \cup(\beta(A) \cap B)=\emptyset$, or equivalently iff $A \cap \theta(B)=\emptyset$.

4.3 Immersions from $\mathbb{Z}^{n}$ into $\mathbb{H}^{n}$

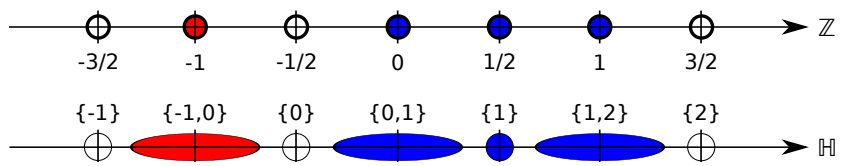

Fig. 20: Bijection between $(\mathbb{Z} / 2)$ and $\mathbb{H}^{1}$.

Now let us define the bijection between $(\mathbb{Z} / 2)^{n}$ and $\mathbb{H}^{n}$ (see Figure 200 on which we will base our immersions into Khalimsky grids. Let $\mathcal{H}:(\mathbb{Z} / 2) \rightarrow \mathbb{H}^{1}$ be the application such that:

$\forall z \in(\mathbb{Z} / 2), \mathcal{H}(z)=\left\{\begin{array}{l}\{z+1 / 2\} \text { if } z \in(\mathbb{Z} / 2) \backslash \mathbb{Z}, \\ \{z, z+1\} \text { if } z \in \mathbb{Z},\end{array}\right.$

from which we deduce naturally the application $\mathcal{H}_{n}$ : $\left(\frac{\mathbb{Z}}{2}\right)^{n} \rightarrow \mathbb{H}^{n}$ such that:

$$
\forall z \in(\mathbb{Z} / 2)^{n}, \mathcal{H}_{n}(z)=\otimes_{i \in \llbracket 1, n \rrbracket} \mathcal{H}\left(z_{i}\right),
$$

where for two arbitrary sets $A$ and $B, A \otimes B:=\{a \times$ $b ; a \in A, b \in B\}$ with $\times$ the Cartesian product; note that elements of $\mathbb{Z}^{n}$ are transformed into $n$-faces of $\mathbb{H}^{n}$. In the sequel, we will denote by $\mathcal{Z}_{n}$ the inverse of the bijection $\mathcal{H}_{n}$.

Note that another "natural" bijection from $(\mathbb{Z} / 2)^{n}$ to $\mathbb{H}^{n}$ is possible, transforming elements of $\mathbb{Z}^{n}$ into vertices of $\mathbb{H}^{n}$; however, this transformation is not convenient to define topological boundaries of immersions of subsets of $\mathbb{Z}^{n}$, and then will not be developed further in this paper.

The first possible immersion of a given binary image $u_{\text {bin }}: \mathbb{Z}^{n} \rightarrow\{0,1\}$ (see Figure 21) would be a 1-1 correspondence from $\mathbb{Z}^{n}$ to $\mathbb{H}^{n}$ since it is bijective. However, we can see in Figure 22 that this approach does not preserve the structure of the initial image by any opening or closing 131, and then it is not interesting as a representation of the initial signal. Two other immersions, based on $\mathcal{H}_{n}$, are well-known in digital topology 43]: the miss-transform $\operatorname{Int}\left(\alpha\left(\mathcal{H}_{n}(X)\right)\right)$ and the

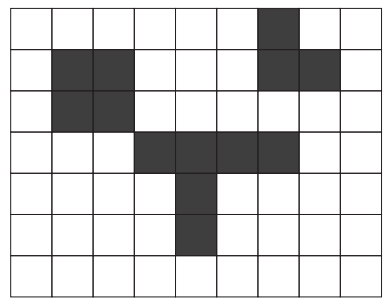

Fig. 21: A binary 2D digital image $u_{\text {bin }}$, that we can identify to the digital set $X=\left[u_{\text {bin }}=1\right]$ [43] (page 31).
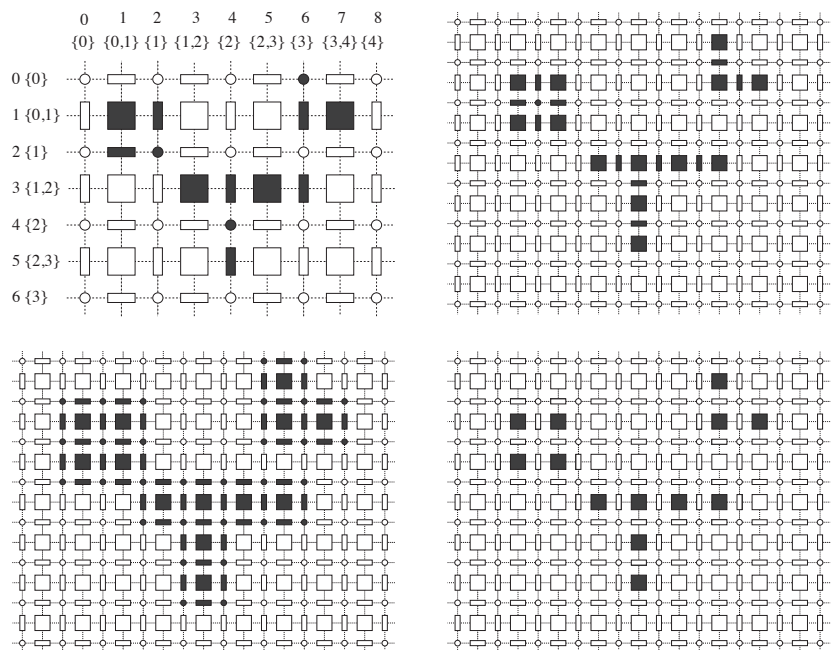

Fig. 22: Different immersions [43,26] of $X$ into $\mathbb{H}^{2}$ : in the raster scan order, the direct immersion, the misstransform, the hit-transform, and $\mathcal{H}_{n}(X)$.

hit-transform $\alpha\left(\mathcal{H}_{n}(X)\right)$, depicted in Figure 22, which both lead to regular sets. By construction, their connected components are respectively open sets for the miss-transform, and closed sets for the hit-transform. The last immersion in Figure 22 has the property to be bijective and to given open sets, but not regular ones. Note that we will usually refer to the hit-transforms and miss-transforms as span-based immersions.

\subsection{Relation between DWCness and AWCness}

Now, let us recall the well-known relation between DWCness of a set and AWCness of its immersion: for any digital subset $X$ of $\mathbb{Z}^{n}$, it is well-known [26] that when $n \in\{2,3\}$, the DWCness of $X$ is equivalent to the AWCness of the span-based immersion, that is, the hitor the miss-transform, of $X$. Let us recall the reasoning between these equivalences.

On Figure 23 the middle of the subfigures represents the restriction of a set to a $2 \mathrm{D}$ block in $\mathbb{Z}^{2}$ (the white points correspond to the foreground), the left of 


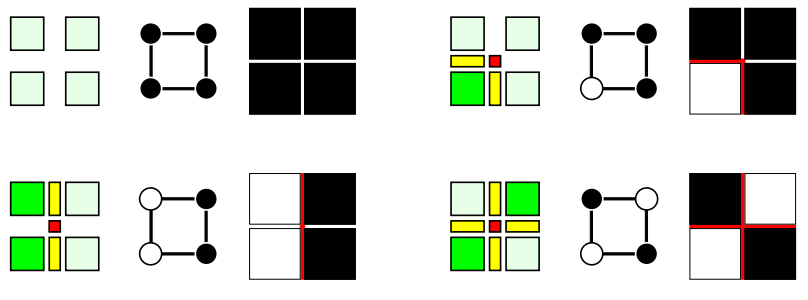

Fig. 23: Set of local configurations in 2D.

the subfigures represents the representation in Khalimsky grids of this same set up to $\mathcal{H}_{n}$ (the foreground is depicted by the green squares and the boundary is depicted by the yellow edges and the red point), and the right of the subfigures represents the continuous analog of the restriction of this set in $\mathbb{R}^{2}$ (the foreground is in white and the boundary is in red).

In the raster scan order, we observe then the following possibilities by comparing the first two columns of the subfigures:

1. if the restriction of the set is made of four black points, that is, no point of $X$ belongs to the block, and then there is no boundary in this part of the Khalimsky grid, we have then nothing to prove,

2 . if the restriction of the set is made of only one point, we can observe that the red point belonging to the discrete boundary has only two neighbors into the boundary: the two yellow edges,

3 . if this resctriction is made of two 4-adjacent white points, the red point belonging to the discrete boundary has one more time two yellow edges as neighbors into the boundary,

4. if this restriction is made of two white points which are 8 -adjacent but not 4 -adjacent, that is, when we have a critical configuration, then we obtain that the red point has four neighbors, the four yellow edges.

Then the red points of the boundary of the representation of the set in Khalimsky grids admit only two neighbors iff the set is digitally well-composed. Since the yellow edges admit always two neighbors, because a boundary is closed (and then contains its vertices in the Khalimsky grid) by construction, we obtain finally that every set which is AWC is DWC and conversely in 2D. Note that Figure 23 also illustrates the equivalence between DWCness and CWCness in 2D.

Looking at Figure 24, with the same reasoning as for the $2 \mathrm{D}$ case, we can see that there is no critical configurations in the restriction $X \cap S$, where $S$ is any 3D block in $\mathbb{Z}^{3}$, if and only if the boundary $\partial \mathcal{I}(X)$ (made of green squares, yellow edges, and red points) of the span-based immersion $\mathcal{I}(X)$ (such that white points in
$\mathbb{Z}^{3}$ become blue cubes) of $X$ is locally a simple closed curve. On the contrary, in the cases containing one or more $2 \mathrm{D}$ critical configurations or a $3 \mathrm{D}$ critical configuration, $\partial \mathcal{I}(X)$ is not locally a simple closed curve: it contains a "pinch" at a yellow edge in the case of a $2 \mathrm{D}$ critical configuration and at a red point in the case of a 3D critical configuration. Note that the cases that we can obtain by complementarity have been omitted since well-composedness is self-dual. This gives the intuition of why AWCness and DWCness are equivalent in 3D.

Boutry [26] asserted in his Ph.D. thesis that this equivalence is still true when $n \geq 4$ but this still remains a conjecture.

\subsection{Well-composedness on polytopal complexes}

As defined in Stelldinger's book [168, a polytopal complex in $\mathbb{R}^{n}$ is a set of convex polyhedra in $\mathbb{R}^{n}$, called polytopes, such that every face of each polytope belongs to this complex, and such that for any two faces of the complex, their intersection is a common face of both these two faces.

The dimension of a polytope is the maximum number of contained independent vectors after translating the polytope so that it covers the origin, and a polytope of dimension $m \geq 0$ is called a $m$-cell. The dimension of a polytopal complex is the maximal dimension of its polytopes.

Two polytopes of a complex are said $m$-adjacent if their intersection is a $m^{\prime}$-polytope with $m^{\prime} \geq m$. Two polytopes are adjacent iff they are adjacent for some $m$. They are incident iff they are adjacent and of different dimensions (then one polytope is a face of the other). A complete polytopal complex of dimension $m$ is a polytopal complex where each polytope of dimension $m^{\prime}<m$ is incident to at least one polytope with dimension $m$.

A polytopal complex is called well-composed iff it is complete, of dimension $n$, and any two adjacent $n$ polytopes are $(n-1)$-adjacent. A set in $\mathbb{R}^{n}$ is said well-composed iff there exists a well-composed polytopal complex such that the union of its polytopes is equal to this set.

According to Stelldinger [168], this definition extends the ones of Latecki [100,102] and Wang and Bhattacharya [183] for arbitrary cell complexes in any dimension.

However, it seems that the polytopal complex such as depicted in Figure 25 made of three edge-connected unit squares depicting a "L", plus their faces, depicts a 


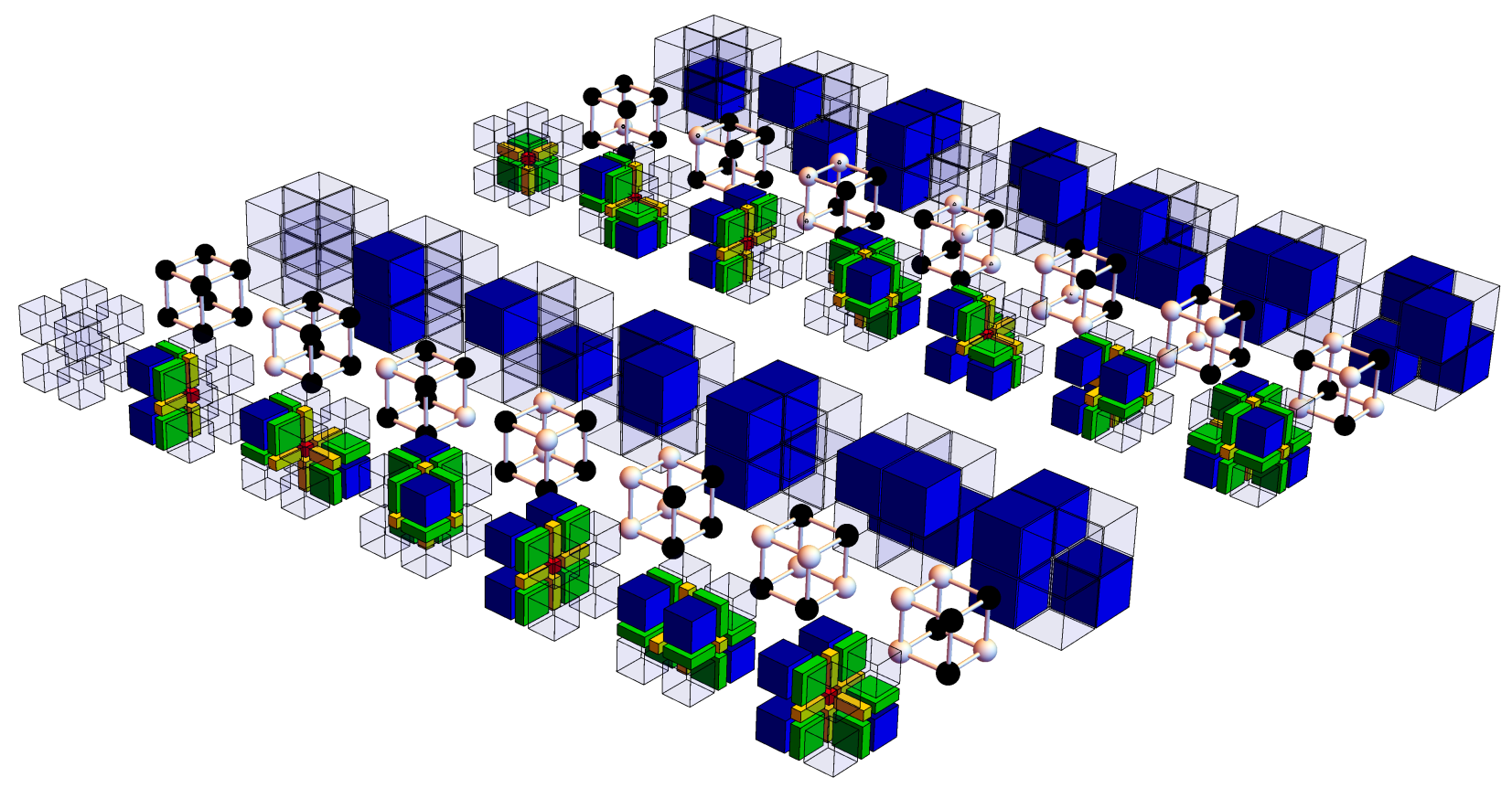

Fig. 24: A set of local configurations in 3D.

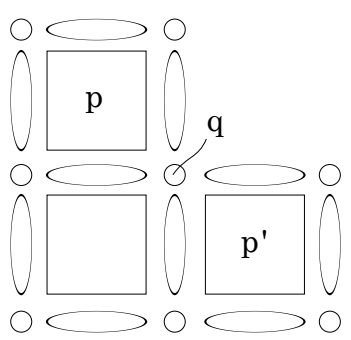

Fig. 25: A polytopal complex which would not be wellcomposed according to Stelldinger [168]; see text for details.

polytopal complex which would be well-composed according to Latecki, since the boundary of the complex is a simple closed curve. It would not be well-composed according to Stelldinger, since this set contains two squares $p$ and $p^{\prime}$ which share a vertex $q$, and then are adjacent, but which do not share any edge. The definition of Latecki and Stelldinger then seems not to be equivalent.

A new definition of well-composedness for polytopal complexes could then follow the one of Boutry [26] about well-composedness of arbitrary grids in $n$-D: a polytopal complex could be said to be well-composed iff for any two different $n$-polytopes $p$ and $q$ incident to a common $m$-polytope $a, 0 \leq m \leq n-1$, there exists a sequence $\Pi=\left(p=f_{n}^{0}, \ldots, q=\bar{f}_{n}^{k}\right), k \geq 1$, of $n$-faces such that for any $i \in \llbracket 0, k-1 \rrbracket, f_{n}^{i}$ and $f_{n}^{i+1}$ share ex- actly a $(n-1)$-face and such that each $f_{n}^{i}, i \in \llbracket 0, k \rrbracket$, is incident to $a$.

However, note that even this way, this definition is not equivalent to the one of Latecki, since the immersion into the Khalimsky grid of a secondary critical configuration in 3D would be well-composed, this definition being not self-dual.

\section{Other flavours of WCnesses}

We have seen definitions of well-composedness in the digital sense in $\mathbb{Z}^{n}$ and in the Alexandrov sense, let us now present the other definitions of well-composednesses that exist nowadays: the well-composedness based on the equivalence of connectivities or EWCness and its relationship with DWCness, the continuous well-composedness or CWCness and its relationship with DWCness in $3 \mathrm{D}$ and with AWCness in $n$-D, and well-composedness on arbitrary grids or $\mathcal{A G}$-well-composedness in $n$-D.

\subsection{Definition of EWCness in $n$-D}

Let us recall the $n$-D extension of well-composedness based on the equivalence of connectivities proposed in [29]: let $X$ be a digital set in $\mathbb{Z}^{n}$, then $X$ is said to be $E W C$ or well-composed based on the equivalence of its connectivities iff the two following conditions hold: 


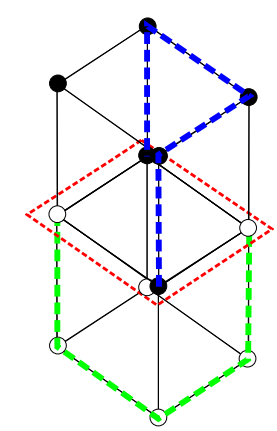

Fig. 26: EWCness does not imply DWCness in $n$-D $(n \geq$ $3)$.

- any of its $2 n$-component is also one of its $\left(3^{n}-1\right)$ components and vice versa,

- any $2 n$-component of $X^{c}$ is also a $\left(3^{n}-1\right)$-component of $X^{c}$ and vice versa.

We can underline that this definition is clearly selfdual, and since the connectivity does not matter for this class of sets, we will sometimes say (when the context is clear) that their connectivities and the ones of their complement in $\mathbb{Z}^{n}$ are "equivalent". Also, this definition is the natural extension of the one of Latecki in 104 for $2 \mathrm{D}$ sets.

\subsection{DWC implies EWC in $n$-D}

Let us recall that EWCness is a global property, since it is based on connected components, and that DWCness is based on local properties, that is, there is no critical configurations. That shows that the link between DWCness and EWCness is not so obvious. However, as presented for the first time in $n$-D in [29] and proved in 26, for any digital subset $X$ of $\mathbb{Z}^{n}$, if $X$ is DWC, then $X$ is EWC.

Recall [29,26] that the converse is not true in 3D and beyond (see Figure 26): a $3 \mathrm{D}$ subset of $\mathbb{Z}^{n}$ can be EWC without being DWC, since the $\left(3^{n}-1\right)$-components and the $2 n$-components of this set are equal, but it contains a $2 \mathrm{D}$ critical configuration and then is not DWC.

\subsection{Definition of CWCness in $n$-D}

The first definition of 3D well-composedness, based on manifoldness, has been first introduced in 1997 [100]. Then, it has been extended to $n$-D in 2000 [102], and renamed as "continuous well-composedness" in 2015 29 to distinguish it from EWCness and DWCness. Before we recall this $n$-D extension, let us introduce some mathematical background. According to Latecki et al. [100.

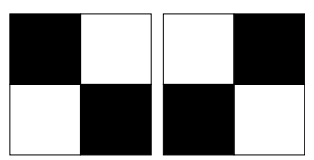

Fig. 27: The continuous analogs of 2D critical configurations in $\mathbb{R}^{2}$.

102 , the continuous analog $\mathrm{CA}(p)$ of a point $p \in \mathbb{Z}^{n}$ is the closed unit cube centered at this point with faces parallel to the coordinate planes: $\mathrm{CA}(p)=\left\{q \in \mathbb{R}^{n} ; \| p-\right.$ $\left.q \|_{\infty} \leq 1 / 2\right\}$, where $\|x\|_{\infty} \equiv \max \left\{x_{i} ; i \in \llbracket 1, n \rrbracket\right\}$ for any $x \in \mathbb{R}^{n}$, and the continuous analog $C A(X)$ of a digital set $X \subset \mathbb{Z}^{n}$ is the union of the continuous analogs of the points belonging to the set $X: \mathrm{CA}(X)=$ $\bigcup_{p \in X} \mathrm{CA}(p)$. This way, a digital subset $X \subset \mathbb{Z}^{n}$ is said well-composed in the continuous sense [100,102 29 , or shortly $C W C$, iff the boundary of the continuous analog $\operatorname{bdCA}(X)$ of this set is a $(n-1)$-manifold, that is, if for any point $p \in X$, the (open) neighborhood of $p$ in $\operatorname{bdCA}(X)$ is homeomorphic to $\mathbb{R}^{(n-1)}$. Note that this definition is self-dual: for any $X \subset \mathbb{Z}^{n}$, $\operatorname{bdCA}(X)=\operatorname{bdCA}\left(X^{c}\right)$ and then $X$ is well-composed iff $X^{c}$ is well-composed.

In [100], Latecki also introduced a characterization of 3D continuous well-composedness using $m$-adjacencies: two points are said 6-adjacent (6-neighbors) iff their continuous analog share a face, 18-adjacent (18neighbors) iff their continuous analogs share a face or an edge, and 26-adjacent (26-neighbors) iff their continuous analogs share a face, an edge, or a corner (of a unit cube centered at a point of $\mathbb{Z}^{3}$ ). Then, with $X$ a digital subset of $\mathbb{Z}^{3}, X_{1}=X$ and $X_{0}=X^{c}, X$ is CWC iff the two following conditions hold for $\kappa \in\{0,1\}$ :

- for every two 18-adjacent points $x$ and $y$ in $X_{\kappa}$, there exists a 6 -path joining $x$ to $y$ into $\mathcal{N}_{18}(x) \cap \mathcal{N}_{18}(y) \cap$ $X_{\kappa}$

- for every two 26-adjacent points $x$ and $y$ in $X_{\kappa}$, there exists a 6 -path joining $x$ to $y$ into $\mathcal{N}_{26}(x) \cap \mathcal{N}_{26}(y) \cap$ $X_{\kappa}$.

This way, we clearly understand that local 18/26-connectivities in CWC sets imply 6-connectivity, and then that CWCness implies EWCness.

\subsection{DWCness is equivalent to CWCness in $2 \mathrm{D} / 3 \mathrm{D}$}

As proved by Latecki et al. [104,100, a digital subset $X \subset \mathbb{Z}^{2}$ contains a critical configuration $\left(\begin{array}{ll}1 & 0 \\ 0 & 1\end{array}\right)$ or its dual $\left(\begin{array}{ll}0 & 1 \\ 1 & 0\end{array}\right)$ iff its continuous analog contains the pat- 

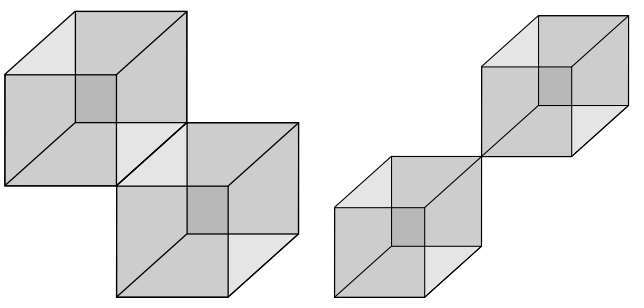

Fig. 28: The continuous analogs of primary $2 \mathrm{D} / 3 \mathrm{D}$ critical configurations in $\mathbb{R}^{3}$.

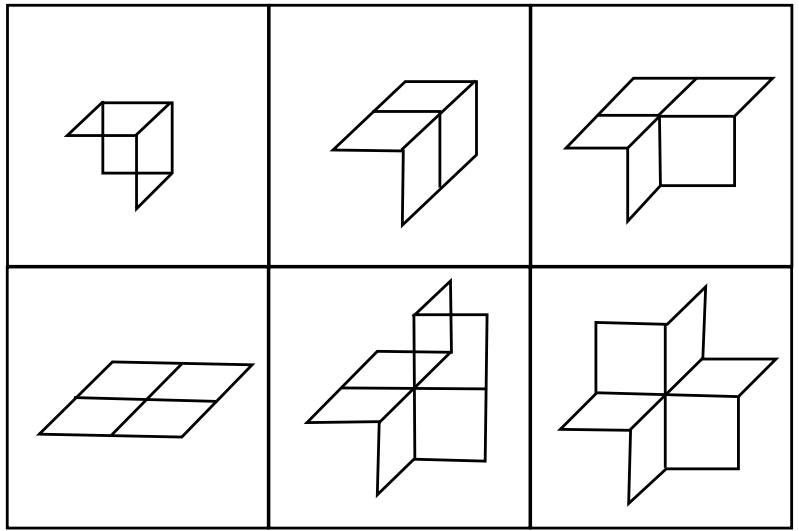

Fig. 29: The six possible configurations [100] at a corner point in a $3 \mathrm{D}$ well-composed set.

tern shown in Figure 27, that is, two squares sharing a vertex.

In the same manner, in $3 \mathrm{D}$, a digital subset $X \subset \mathbb{Z}^{3}$ contains a critical configuration $\left(\begin{array}{ll}1 & 0 \\ 0 & 1\end{array}\right)$ or $\left(\begin{array}{ll|ll}0 & 0 & 0 & 1 \\ 1 & 0 & 0 & 0\end{array}\right)$ iff its continuous analog contains one of the patterns shown in Figure 28 (modulo 90 degrees rotations and translations), that is, two cubes sharing a edge or two cubes sharing a vertex. In other words, DWCness and CWCness are equivalent in $2 \mathrm{D} / 3 \mathrm{D}$.

The complete proof [100] (pp. 166-167) relies on the fact that any set containing one of these critical configurations contains a "pinch" such that at these critical locations, no point of the boundary owns an open neighborhood homeomorphic to an open disk, and then to $\mathbb{R}^{2}$. Conversely, if the set $S$ does not contain any critical configuration of any type, then at each point belonging to the interior of a face, any neighborhood which is small enough will be homeomorphic to an open disk, at any point belonging to the interior of the union of two adjacent faces of the boundary sharing an edge; the neighborhood of this point is homeomorphic to an open disk (whether the two faces are parallel or perpendicular). At the corners of the faces included in the boundary, only 6 configurations are possible (see Figure 291. In the six cases the corner admits a neighbor-

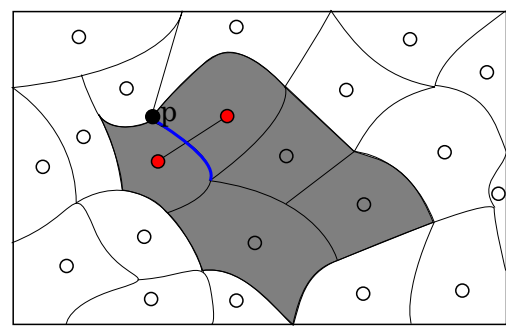

Fig. 30: Definition of 2D well-composedness on 2D arbitrary grids.

hood homeomorphic to an open disk, which concludes the proof of Latecki. However, we can denote that this study has been processed case-by-case and then seems difficult to be extended to higher dimensions.

\subsection{AWCness vs. CWCness in $n$-D}

In digital topology, its is generally admitted that in $2 \mathrm{D}$ and $3 \mathrm{D}$ a digital set $X \subseteq \mathbb{Z}^{n}$ is continuous well-composed, that is, the boundary of its continuous analog $\operatorname{bdCA}(X)$ is a $(n-1)$-manifold, iff its span-based immersion in the Khalimsky grids is well-composed in the sense of Alexandrov, that is, its boundary is a disjoint union of discrete $(n-1)$-surfaces. However, the study of $n$-D well-composedness is yet in its infancy, as shown by the conjecture of Boutry [26] in his thesis, asserting that CWCness and AWCness are equivalent in $n$-D.

\subsection{Well-composedness on arbitrary grids}

According to Wang and Battacharya 183, we can extend the definition of well-composedness coming from the rectangular grids to arbitrary grids in $2 \mathrm{D}$ in the following manner. We assume that we have a (locally finite) arbitrary grid system of (closed) pixels paving the topological space $\mathbb{R}^{2}$ such that the boundary of each pixel is a simple closed curve (or Jordan curve) as depicted in Figure 30

A set $X$ of pixel is then said well-composed iff for any point $p$ belonging to the boundary of $X$, the set of pixels of $X$ containing $p$ is edge-connected [183, which means that for any two pixels in this set, there exists a sequence of pixels of this set going from the first to the second such that two consecutive elements share an edge. Figure 30 depicts a well-composed set in dark gray: at each boundary point $p$ of $X$, the set made of the pixels containing $p$ in $X$ is edge-connected (the edge shared by the two pixels is in blue). Indeed, in the case of rectangular pixels, we obtain that a set $X$ is wellcomposed in the sense of Latecki 104 iff 8-connectivity 
(vertex-connectedness) implies 4-connectivity (edge-connectedness). A particular grid system is the hexagonal grid where every set of pixels is well-composed [183], which is obviously not the case of the rectangular grid.

Serra and Kiran 159 worked on this last topic: $\mathbb{R}^{n}$ is partitioned into a set of regular open sets, called a tessellation, and the complement in $\mathbb{R}^{n}$ of its union, called the net. In this framework [159], they recall an observation of Fedorov [58] which states that the only possible tessellations inherited from a Voronoï grid system such that its elements are identical (up to a translation) are in 2D the square and the hexagonal grid systems, and in 3D the cube, the hexagonal prism, the two elongated and rhombic dodecahedra, and the truncated octahedron; this last one is the Voronoï polyhedron of a body-centered cubic grid, also called BCC grid [172, and is well-known for its guarantees in matter of topology preservation.

Among them, only the hexagonal grid system and the truncated octahedron verify that any two elements of the tessellation, such that their adherence intersect, share a face of dimension $(n-1)$, i.e. an edge in 2D and a (2D) face in 3D. In other words, any (finite) set of elements of these tessellations is strongly adjacent: there exists a small open disk/ball in 2D/3D such that any intersection of adherences of two adjacent elements of $X$ contains this disk/ball.

Then the link between the works of Wang and Battacharya [183] and Serra and Kiran [159] is that strong adjacency is similar to well-composedness on arbitrary grids. Effectively, if we consider a tessellation and an arbitrary grid system which are isomorphic in the sense that they have the same topological structure, every subset of this tessellation which is strongly adjacent has its isomorph in the arbitrary grid system which is wellcomposed, and conversely. However, remark that strong adjacency is based on open sets and well-composedness on arbitrary grids is based on closed sets.

In 2016, Boutry [26] extended this definition of wellcomposedness to (locally finite) arbitrary grids, which justifies our proposition to rename it $\mathcal{A G}$-well-composedness. Let us assume that we have some partition $\left\{\mathcal{V}_{i}\right\}_{i \in I}$ of $\mathbb{R}^{n}$ such that the boundary of each $\mathcal{V}_{i}$ for $i \in I$ is a connected $(n-1)$-manifold (see $75,110,93,3$, for complements about the Jordan-Brouwer theorem in $n$-D). The elements of $\left\{\mathcal{V}_{i}\right\}_{i \in I}$ are then called the voxels. Then, any set $X$ of voxels is said $\mathcal{A} \mathcal{G}$-well-composed (AGWC) iff for any face $f$ of dimension $k \in \llbracket 0, n-1 \rrbracket$ belonging to the boundary of $X$, the set $Y$ of voxels of $X$ containing $f$ (respectively, the set $Y^{\prime}$ of voxels not in $X$ and containing $f$ ) are face-connected, which means

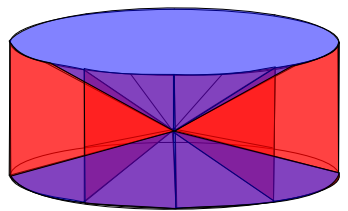

Fig. 31: A set $X$ where the set of voxels in $X$ (in red) containing the boundary point $p$ (at the center of the cylinder) is face-connected and such that the set of voxels in the complement of $X$ (in blue) is not faceconnected [26].

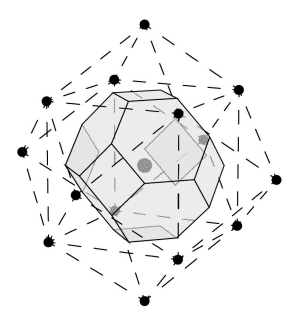

Fig. 32: A truncated octahedra (p. 13 [116]).

that for any two voxels in this set $Y$ (respectively, $Y^{\prime}$ ), there exists a sequence of voxels of this same set going from the first to the second such that two consecutive elements share a face of dimension $(n-1)$.

Note that self-duality in the $n$-dimensional case is ensured because of the double condition, the first relative to $X$ and the second relative to the complement of $X$ (see Figure 31). Its equivalence (or not) with CWCness has not yet been studied.

This way, we obtain that, in the grid system made of truncated octahedra (see Figure 32 covering $\mathbb{R}^{3}$, every set of voxels is well-composed. Effectively, as stated by L. Mazo in his thesis [116], two voxels in such a grid system share either a face of dimension 2 or nothing. This means that two voxels which belong to a set $X$ and which are connected in this set $X$ are face-connected in this same set $X$, and that the converse is true for $X^{c}$. This way, every set in such a space is well-composed. This adjacency is known as $2\left(2^{n}-1\right)$ adjacency in $n$-D (6-adjacency in $2 \mathrm{D}, 14$-adjacency in $3 \mathrm{D}$, and so on), but exhibits a strong anisotropy (see Figure 2.7 into [116]) on the graph of the covered domain.

\section{Extending well-composedness to images}

In this section, we recall first the seminal definition of well-composedness for gray-level 2D images. Then, we show how CWCness, DWCness, and EWCness have been extended to $n$-D gray-level images. Then, we recall the definition of EWCness, CWCness, DWCness 
and AWCness for interval-valued maps. We conclude this section by recalling the definition of well-composed multi-label images.

6.1 Seminal definition of 2D gray-level well-composed images

A 2D gray-level image is a pair $I=\left(\mathbb{Z}^{2}, u\right)$ where $u: \mathbb{Z}^{2} \rightarrow \llbracket 0,255 \rrbracket$ is a mapping from $\mathbb{Z}^{2}$ to $\llbracket 0,255 \rrbracket$ (or more generally from $\mathbb{Z}^{2}$ to any finite set supplied with a total order relation). This image $I$ is generally identified with its mapping $u$ since these two concepts are equivalent. Then, we can apply a very straightforward operation called binarization of a gray-level image relatively to a given threshold. Given a gray-level image $u: \mathbb{Z}^{2} \rightarrow \llbracket 0,255 \rrbracket$ and a threshold $\lambda \in \mathbb{Z}$, the resulting binarization of $u$ relatively to $\lambda$ is equal to the binary image $u_{\text {bin }}: \mathbb{Z}^{2} \rightarrow\{0,1\}$ defined for any $p \in \mathbb{Z}^{2}$ such that $u_{\text {bin }}(p)=1$ if $u(p) \geq \lambda$ and $u_{\text {bin }}(p)=0$ if $u(p)<\lambda$.

A 2D gray-level image is said to be well-composed [102] iff all its binarizations are well-composed. Furthermore, Latecki [102] introduced a characterization of 2D well-composed gray-level images: a gray-level image $I=\left(\mathbb{Z}^{2}, u\right)$ is well-composed iff for any restriction of $u$ to a $2 \times 2$ square, denoted by $\left(\begin{array}{ll}a & b \\ c & d\end{array}\right)$, the diagonal intervals have a non-empty intersection:

$$
[\min (a, d), \max (a, d)] \cap[\min (b, c), \max (b, c)] \neq \emptyset .
$$

Note that this principle of binarization follows the idea of cross-section topology [120,21,17,18], which has been showed to be much convenient to extend set operators to gray-level operators in mathematical morphology [25, 156, 79, 80, 144, 16].

\subsection{Well-composed gray-level $n$-D images}

Based on the same principle as binarizations, threshold sets [120,21,17,18, have allowed to extend well-composedness from sets to gray-level images. Let $\mathcal{D} \subseteq \mathbb{Z}^{n}$ be either $\mathbb{Z}^{n}$ (theoretical case) or a bounded hyperrectangular subdomain of $\mathbb{Z}^{n}[26]$ (practical case). Let $u: \mathcal{D} \rightarrow \mathbb{R}$ be a gray-level image, and let $\lambda \in \mathbb{R}$ be a given threshold. Then, a large upper threshold set is defined as:

$$
[u \geq \lambda]=\{x \in \mathcal{D} ; u(x) \geq \lambda\}
$$

a strict upper threshold set is defined as:

$$
[u>\lambda]=\{x \in \mathcal{D} ; u(x)>\lambda\}
$$

a large lower threshold set is defined as:

$$
[u \leq \lambda]=\{x \in \mathcal{D} ; u(x) \leq \lambda\},
$$

and a strict lower threshold set is defined as:

$$
[u<\lambda]=\{x \in \mathcal{D} ; u(x)<\lambda\} .
$$

Then, a gray-level image $u: \mathcal{D} \subseteq \mathbb{Z}^{n} \rightarrow \mathbb{R}$ is said $X$ $W C$, where $X$ belongs to $\{E, D, C\}$, iff for every threshold $\lambda \in \mathbb{R}$, all the threshold sets of $u$ are $X$-WC. Obviously, the relations between these 3 different flavours of WCnesses for images hold in the same way as for sets, thanks to cross-section topology.

As noticed in [26, in the case of a gray-level image defined on a bounded hyperrectangle, we are able to detect the digital well-composedness of this image using only the upper (respectively, lower) threshold sets: a gray-level image $u: \mathcal{D} \rightarrow \mathbb{R}$ is digitally well-composed iff for any $\lambda \in \mathbb{R}$ the threshold set $[u \geq \lambda]$ is digitally well-composed or equivalently iff for any $\lambda \in \mathbb{R}$ the threshold set $[u \leq \lambda]$ is digitally well-composed. Furthermore, the characterization [29] of gray-level digitally well-composed images holds. A gray-level image $u: \mathcal{D} \subset \mathbb{Z}^{n} \rightarrow \mathbb{R}$ is digitally well-composed iff for any block $S \in \mathcal{B}(\mathcal{D})$ such that $\operatorname{dim}(S) \geq 2$ and for any pair of points $\left(p, p^{\prime}\right) \in S \times S$ such that $p^{\prime}=\operatorname{antag}_{S}(p)$, the following relation is true:

$\operatorname{intvl}\left(u(p), u\left(p^{\prime}\right)\right) \cap \operatorname{Span}\left\{u\left(p^{\prime \prime}\right) \mid p^{\prime \prime} \in S \backslash\left\{p, p^{\prime}\right\}\right\} \neq \emptyset$.

We recall that for any $a, b \in \mathbb{R}$,

$$
\operatorname{intvl}(a, b):=[\min (a, b), \max (a, b)],
$$

and that for any set $A$ of real numbers,

$$
\operatorname{Span}(A):=[\min (A), \max (B)] .
$$

Also, note that an algorithm able to verify the digital well-composedness of a gray-level image has been proposed in [26].

\subsection{WCnesses for interval-valued maps}

Before recalling what is well-composedness for intervalvalued maps, let us reintroduce some mathematical background related to set-valued theory [8]. A set-valued map $U: \mathcal{D} \subseteq X \rightsquigarrow Y$ is a function from a topological space $X$ to a topological space $Y$ such that for any $p \in X, p \in \mathcal{D} \Leftrightarrow U(p) \neq \emptyset(\mathcal{D}$ is called the domain of $U)$ and such that $\forall p \in \mathcal{D}, U(p) \subseteq Y$. Then, an intervalvalued map $U: \mathcal{D} \subseteq X \rightsquigarrow Y$ is a set-valued map such that for any $p \in \mathcal{D}, U(p)$ is an interval of the topological space $Y \subseteq \mathbb{R}$, that is, $U(p)$ can be written $[a, b] \cap Y$ for some $a, b \in \mathbb{R}$ such that $a \leq b$. 


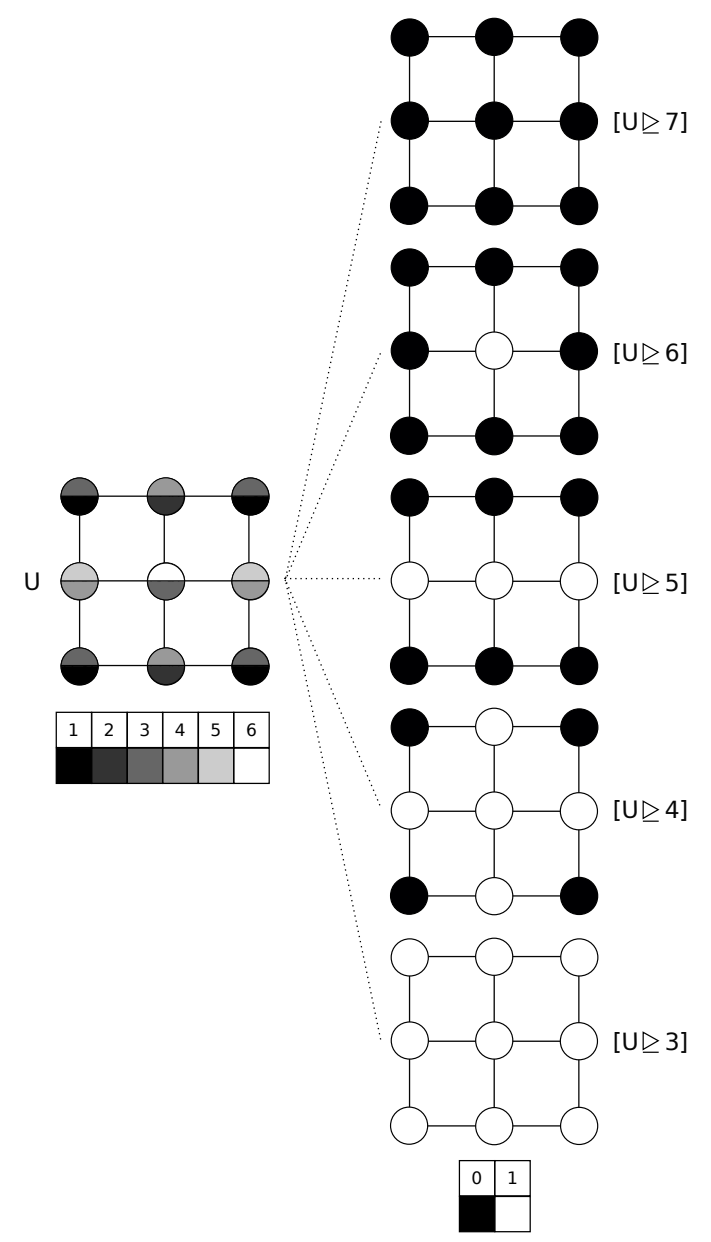

Fig. 33: A family of (large upper) threshold sets $\{[U \unrhd$ $\lambda]\}_{\lambda}$ of an interval-valued image $U: \mathbb{Z}^{2} \rightsquigarrow \mathbb{Z}$; we can remark the straightforward inclusion relationship $[U \unrhd$ $\lambda] \subseteq[U \unrhd \lambda-\varepsilon]$ for any $\lambda \in \mathbb{R}$ and $\varepsilon>0$.

Now that we have defined interval-valued maps, we can recall the definition of their threshold sets (see Figures 33 and 34. For a given interval-valued map $U$ : $\mathcal{D} \subseteq X \rightsquigarrow \mathbb{R}$, where $X$ is generally $\mathbb{Z}^{n}$ or $\mathbb{H}^{n}$, and for any $\lambda \in \mathbb{R}$, the following sets:

$$
\begin{aligned}
& {[U \unrhd \lambda]=\{z \in \mathcal{D} \mid \exists v \in U(z), v \geq \lambda\},} \\
& {[U \triangleright \lambda]=\{z \in \mathcal{D} \mid \forall v \in U(z), v>\lambda\},} \\
& .[U \triangleleft \lambda]=\{z \in \mathcal{D} \mid \forall v \in U(z), v<\lambda\}, \\
& {[U \unlhd \lambda]=\{z \in \mathcal{D} \mid \exists v \in U(z), v \leq \lambda\}}
\end{aligned}
$$

are respectively called the large upper, the strict upper, the strict lower, and the large lower threshold sets [127, 63.

Let $U: \mathcal{D} \rightarrow \mathbb{I}_{\mathbb{R}}$, where $\mathbb{I}_{\mathbb{R}}$ is the set of intervals of $\mathbb{R}$, that we will shortly write $U: \mathcal{D} \rightsquigarrow \mathbb{R}$, then, $U$

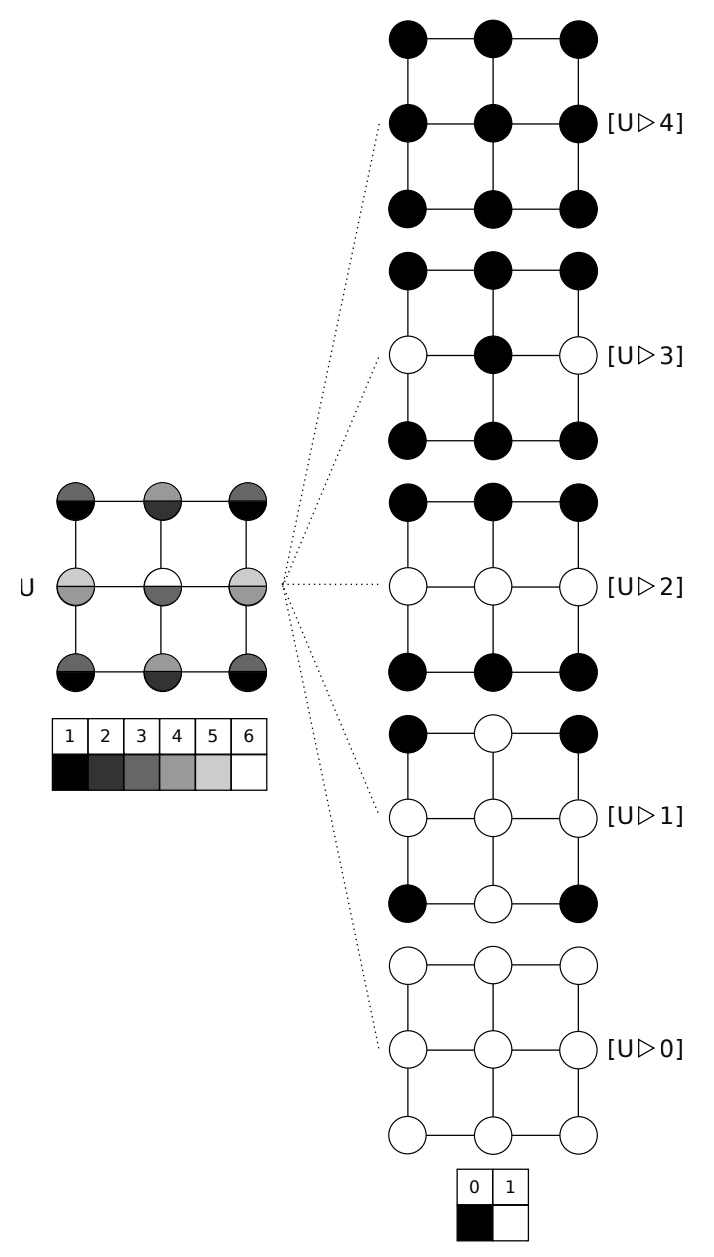

Fig. 34: A family of (strict upper) threshold sets $\{[U \triangleright$ $\lambda]\}_{\lambda}$ of an interval-valued image $U: \mathbb{Z}^{2} \rightsquigarrow \mathbb{Z}$; we can remark the straightforward inclusion relationship $[U \triangleright$ $\lambda] \subseteq[U \triangleright \lambda-\varepsilon]$ for any $\lambda \in \mathbb{R}$ and $\varepsilon>0$.

is said $X-W C$ [127]29], where $X \in\{A, C, D, E\}$, as an interval-valued map iff all its threshold sets are $\mathrm{X}-\mathrm{WC}$.

Also, for an $n$-D interval-valued map $U: \mathcal{D} \subseteq \mathbb{Z}^{n} \rightsquigarrow$ $\mathbb{R}$, the upper bound $\lceil U\rceil$ and the lower bound $\lfloor U\rfloor$ are defined such that for any $p \in \mathcal{D},\lceil U\rceil(p)=\max (U(p))$ and $\lfloor U\rfloor(p)=\min (U(p))$. Then, it is known [29] that an interval-valued map $U: \mathcal{D} \subseteq \mathbb{Z}^{n} \rightsquigarrow \mathbb{R}$ is digitally well-composed iff $\lceil U\rceil: \mathcal{D} \rightarrow \mathbb{R}$ and $\lfloor U\rfloor: \mathcal{D} \rightarrow \mathbb{R}$ are both digitally well-composed.

Note that we have the same relations between AWCness, CWCness, DWCness and EWCness for sets and for gray-level images, except that when we speak about the AWCness of a set, we consider its hit- or misstransform (immersion) in the Khalimsky Grids $\mathbb{H}^{n}$ and when we speak about AWCness of a gray-level image, we consider its span-based immersion. Let us recall that the span-based immersion of a gray-level image $u$ : $\mathcal{D} \subseteq \mathbb{Z}^{n} \rightarrow \mathbb{Z}$ is the interval-valued map $U: \mathcal{D}^{\prime} \equiv$ 


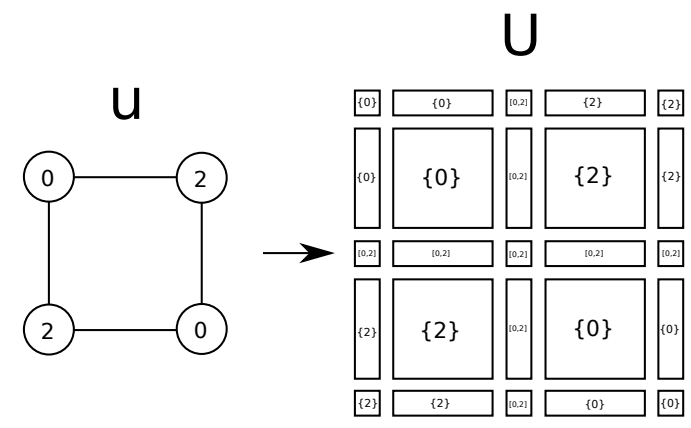

Fig. 35: The span-based immersion of a 2D image.

$\alpha\left(\mathcal{H}_{n}(\mathcal{D})\right) \rightsquigarrow \mathbb{Z}$ defined on the Khalimsky grid such that $\forall z \in \mathcal{D}^{\prime}$ :

$U(h)= \begin{cases}\left\{u\left(\mathcal{Z}_{n}(h)\right)\right\} & \text { if } h \in \mathbb{H}_{n}^{n}, \\ \operatorname{Span}\left\{u\left(\mathcal{Z}_{n}(q)\right) ; q \in \beta(h) \cap \mathbb{H}_{n}^{n}\right\} & \text { otherwise. }\end{cases}$

An example of span-based immersion is given in Figure 35

\subsection{Well-composed multi-label $n$-D images}

Now, considering a set $\left\{c_{1}, \ldots, c_{k}\right\}$ of arbitrary elements $c_{m}$ called labels or colors, the most natural definition of well-composedness for multi-label images, presented first in its digital version in [163], is the following: a multi-label image $u: \mathcal{D} \subseteq \mathbb{Z}^{n} \rightarrow\left\{c_{1}, \ldots, c_{k}\right\}$ is said $X-W C, X \in\{A, C, D, E\}$, iff all the level-sets $\left[u=c_{m}\right] \equiv\left\{x \in \mathcal{D} ; u(x)=c_{m}\right\}, m \in \llbracket 1, k \rrbracket$, are $\mathrm{X}-\mathrm{WC}$.

\section{Producing DWC sets/images}

In this section, we first introduce the different methods that exist nowadays to produce well-composed images, then we develop topological reparations on $\mathbb{Z}^{n}$ and on cubical complexes, and then we recall the main DWC interpolations for sets and images; we finish by recalling existing AWC representations of gray-level images in Khalimsky grids.

\subsection{Preamble}

Two main approaches exist to make a set or an image well-composed on a cubical grid: topological reparations and well-composed interpolations. The first one is called topological reparation, because we consider that we give back to the objects in the image the topological properties they had before the digitization process; mainly, digitized objects should have a boundary which is a $(n-1)$-manifold. The second approach correspond to interpolations, since their restriction to the initial domain is then assumed to be exactly the initial image. However, without constraints, there is no guarantee that the interpolation has the same topology as the initial image. For example, the 1D image • • represents two connected pixels, valued at 1 . One non-constrained interpolation can then be • $\circ \bullet$, where $\circ$ denotes a pixel valued at 0 . The two black points are then disconnected. For this reason, only in-between interpolations are interesting, that is, interpolations such that the secondary pixels have values that are between the values of the primary pixels. Such interpolations have the property of not introducing new extrema in the image. This way, in-between interpolations "preserve" the topology of the initial image.

\subsection{Topological repairing on cubical grids}

Digital images resulting from a convenient digitization of a manifold should be CWC, assuming that the digitization procedure preserves the topology of the initial object. Effectively, real objects, or most of them, have a boundary which is a topological manifold.

However, it is well-known that it is not always the case in image processing: the choice of digitization is not always adapted to the situation, the resolution of the digitization can be too large, and so on. Moreover, it has been shown [171] that even digitization by intersection, which results in well-composed images in $2 \mathrm{D}$ for a sufficient resolution [76], does not provide bordered 3manifolds by reconstruction using cubical voxels, whatever the chosen resolution.

It seems then useful to know how to make digital images CWC in $n$-D. Latecki [101,102] called this procedure "topological repairing", and introduced the first method in $2 \mathrm{D}$ able to do it. As usual, the ones correspond to the object/foreground and the zeros to the background. His method proceeds then by changing the zeros where critical configurations occur into the binary initial image into ones. Also, depending on the neighborhood surrounding the critical configuration and the possible propagation of the critical configuration, a different method is chosen to eliminate the critical configurations in such a neighborhood. This method is translation-invariant and 90 degrees rotation invariant, and guarantees that the number of modifications is minimal.

Then, Siqueira et al. 162,163 proposed a 3D randomized method which produces CWC any 3D binary image. Since no assumption is made on the topology 

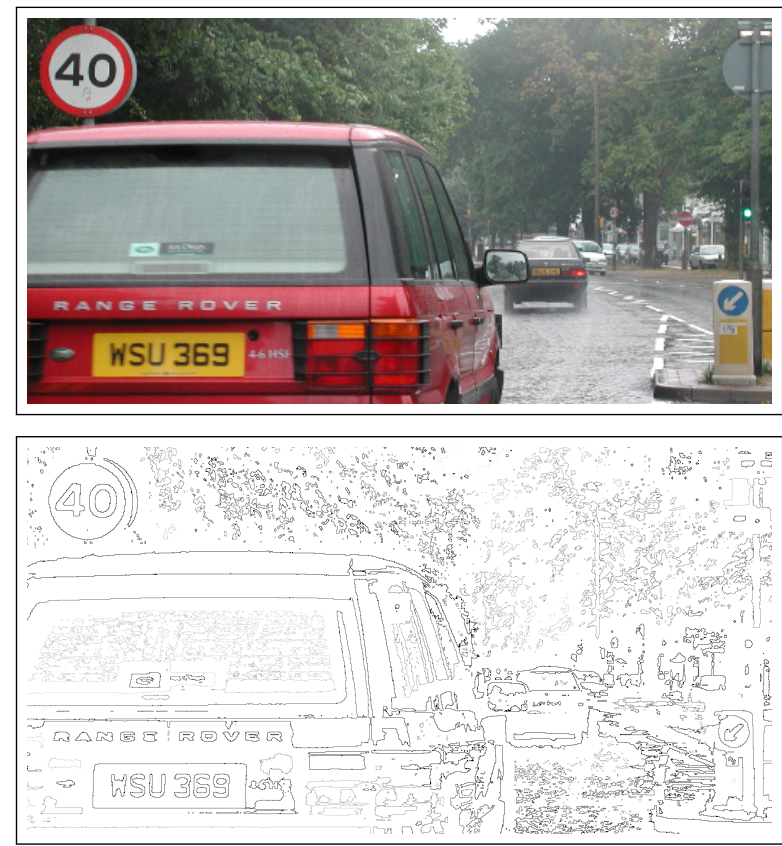

Fig. 36: Hierarchical representation of an image: since component boundaries are simple closed curves on wellcomposed images, two boundaries are either disjoint or in an inclusion relationship; thus, the delimited regions naturally form a tree (actually it is a sub-part of the tree of shapes [63]).

of the initial object, no topological equivalence is ensured, but a theoretical bound ensures that the maximal number of new critical configurations which will appear during the elimination of the $m$ initial configurations is lower than or equal to $m / 2$. Note that this method also works on multi-label images [163, assumed to be the result of a multi-label segmentation; an order of priorities between the different labels is then necessary to decide how to repair the initial image into a well-composed multi-label image.

No method able to make sets CWC in $4 \mathrm{D}$ or to make gray-level images $\mathrm{CWC}$ in $3 \mathrm{D}$ exist nowadays to the best of our knowledge. However, Boutry et al. 28. proposed in 2015 a method able to "repair" in $n$-D any set or gray-level image in such a manner that the resulting signal is DWC, that is, does not contain any critical configuration; this method is based on an "increasing" procedure which avoids oscillations and ensures convergence in linear time w.r.t. the size of the domain of the image. No topological preservation is ensured since no assumption is made on the initial signal. However, it has been observed that the zero-crossings of the DWC reparation of the Laplacian are simple closed curves in $2 \mathrm{D}$, and then provide an hierarchical representation of the objects in the image [28] (see Figure 36.
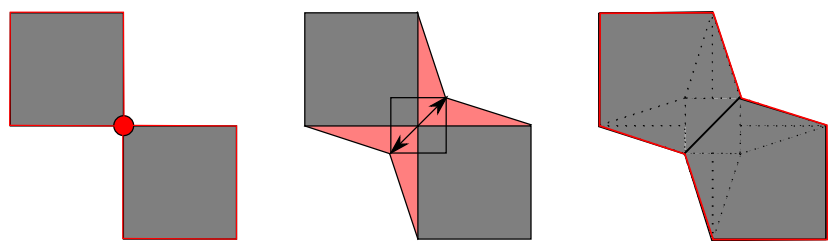

Fig. 37: The equivalent in 2D of the repairing method of Gonzalez-Diaz et al. 67].

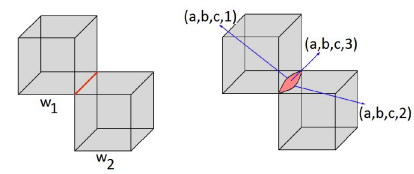

Fig. 38: Repairing of a complex containing a critical edge [67].

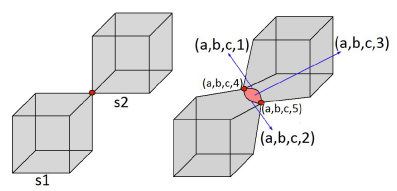

Fig. 39: Repairing of a complex containing a critical vertex [67].

\subsection{Topological repairing of cubical complexes}

Gonzalez-Diaz et al. 67] introduced in 2011 a method able to topologically repair a cubical complex associated with a $3 \mathrm{D}$ binary digital image into a polyhedral complex which is homotopy equivalent and wellcomposed in the sense that the boundary of its underlying polyhedron is a 2-manifold; cohomological information $[71,72,65,66,70$ is then computable directly on this manifold. The proposed (local) method is homotopy preserving, which implies that the resulting cohomological informations can be used to recognition or characterization tasks.

Their method would be this way in $2 \mathrm{D}$ : on a $2 \mathrm{D}$ cubical complex, as shown in Figure 37 the area of the surface of each "critical point" would be "increased" such that there is no more pinch into the boundary of the complex (in dark gray), which would lead to a 2D well-composed polyhedral complex (in dark gray too) whose boundary (in red) is made of simple closed curves.

In $3 \mathrm{D}$, the critical faces, that is, the faces in the combinatorial structure corresponding to the pinch in the underlying polyhedron of the complex, are "stretched" such that the pinch disappears: Figure 38 shows how a critical edge shared by two edge-adjacent cubes $w_{1}$ and $w_{2}$ is replaced by a face of dimension 2 plus two 

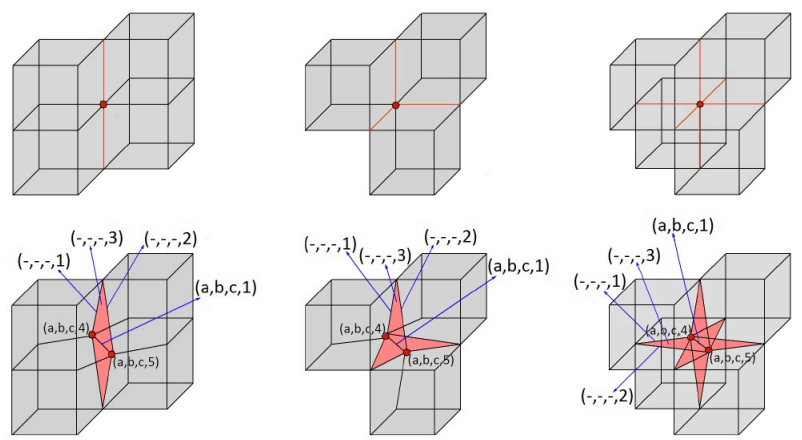

Fig. 40: Repairing of more complex forbidden configurations [67.

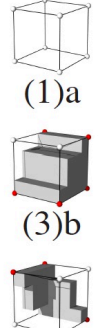

(7)

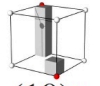

(10)a

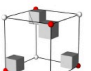

(13)

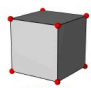

(1) b

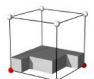

(4)a

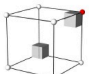

(8)a

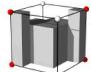

(10)b

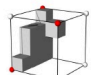

(14)

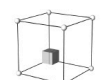

(2)a

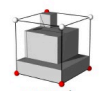

(4)b

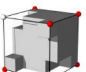

(8)b

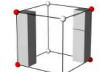

(11)

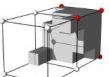

(8) $\mathrm{a}+\mathrm{b}$

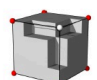

(2)b

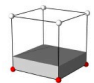

(5)

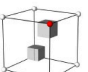

(9)a

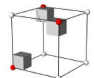

(12)a

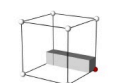

(3)a
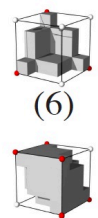

(9)b

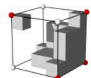

(12)b
Fig. 41: Different configurations using Majority Interpolation [170].

bordering edges, and Figure 39 shows how a critical vertex, shared by two vertex-adjacent cubes $s_{1}$ and $s_{2}$, is replaced by a combinatorial structure made of one 2-face, two bordering edges, and their common vertices. More complicated structures are used to repair the other problematic configurations (see Figure 40). Note that this method is not self-dual since it "expands" the initial complex.

An efficient coding of this family of polyhedral complexes, called ECM representation [68,69], and using 3D cubical images, has been developed to store this family of repaired and well-composed complexes efficiently.

\subsection{DWC interpolations on $\mathbb{Z}^{n}$}

In 1998, Rosenfeld, Kong and Nakamura [152] developed the first well-composed 2D interpolation, that is to say, a method able to compute an image on a larger domain than the one of the initial image, such that its restriction to the initial domain equals the original image and such that the resulting interpolation is wellcomposed.

This method can be decomposed in two steps. First an image magnification [152, which is equivalent to replacing each pixel of the original image by a set of $(k+1) \times(k+1)$ pixels (where $k \geq 1$ is given) of the same value and which replaces the original pixel. Secondly, a modification step removes the critical configurations of the magnified image by changing one of the values of the 4 points of the critical configuration (from 0 to 1 or the converse). Since the magnification process and the modifications are simple deformations [152], they preserve the topology (in the sense that the two images have the same adjacency tree and the same homotopy type), and then the final image is a well-composed image topologically equivalent to the initial one.

Then in 2000, Latecki [102] developed an alternative method to make a $2 \mathrm{D}$ binary image well-composed. This new method is based on the image expansion of Köthe [94, and consists of doubling the resolution of the square grid of the initial image by adding new pixels (the so-called "secondary" pixels) between the initial pixels (the "primary" pixels). A secondary pixel added between two pixels that were edge-connected in the initial grid will take the value of these primary pixels iff they have the same value. Otherwise, they will be labeled as "boundary points". A secondary pixel added at the center of a square of 4 pixels that were vertexconnected in the initial grid will take the value of these pixels iff they all have the same value. Otherwise, they will be labeled as boundary points. Finally, we obtain 3 sets, a set of zeros, a set of ones, and a set of boundary points; each of these sets is well-composed.

Then in 2006, Stelldinger proposed a method called Majority Interpolation 170, shown in Figure 41, which can be seen as a slightly modified 3D extension of the method of Latecki [102, since it is based on a similar counting process. The resulting binary image is always well-composed in the sense that the resulting boundary in the interpolated image is a 2-manifold, but this method is not self-dual.

In 2000, Latecki [102] developed the first gray level well-composed interpolation method in $2 \mathrm{D}$. Using the same image expansion as the one used for the binary interpolation, the new pixels are valued based on bilinear interpolation: a pixel added between 2 primary pixels is valued at the mean of these two pixels, and at the center of a square of primary pixel, the new pixel is set at the mean of the values of these 4 pixels if the restriction of 
the image to these four pixels was well-composed, and at the median of these same values otherwise.

This last method has been slightly modified by Géraud et al. 62 in 2015 where the new pixels added at the center of a square of 4 pixels is always the median of these four primary pixels, since the median is a good solution to make an image well-composed in 2D (and only in 2D [27]). This method does not create any extrema.

We can notice that these gray-level interpolation methods are self-dual in the sense that they do not overemphasize bright components of the dark ones, nor the converse. The counterpart of this powerful property is that the initial images having a integer-based value space, the value space of the new images is $\mathbb{Z} / 4$ for the method of Latecki and $\mathbb{Z} / 2$ for the method of Géraud et al..

As noticed in [27, extending 2D well-composed interpolations to $n$-D is not so easy when we want to ensure self-duality using a local interpolation with usual constraints. Effectively, Mazo [117] developed a method able to interpolate any image in $n$-D into a DWC one [26], based on the connectivity functions [117] where $\varepsilon=1$ corresponds to the max interpolation and $\varepsilon=-1$ corresponds to the min interpolation. Even if this method is initially developed for binary images defined on Khalimsky grids, its extension to $\mathbb{Z}^{n}$ and to gray level images is well known and frequently used. However, this method is not self-dual.

Finally, Boutry et al. [29] introduced in 2015 a $n$-D method able to interpolate in a self-dual and non-local way images into a DWC representation which is "inbetween", that is, which does not introduce any new extrema and, in that sense, preserves the contours. This method first computes a span-based DWC interpolation $U:(\mathbb{Z} / 2)^{n} \rightsquigarrow \mathbb{R}$ of the given image $u: \mathbb{Z} \rightarrow \mathbb{R}$, and applies on it a front propagation algorithm coming from the algorithm of computation of the tree of shapes 63 . which preserves DWCness, to obtain the self-dual DWC representation $u_{\mathrm{DWC}}:(\mathbb{Z} / 2)^{n} \rightsquigarrow \mathbb{R}$. This algorithm is linear in time, deterministic (even if based on a randomized handling of a hierarchical queue), and ensures DWCness in $n$-D, $n \geq 2$. Figure 42 depicts some of the interpolations we have mentioned in this section.

\subsection{AWC representations on $\mathbb{H}^{n}$}

Initially, Géraud and Najman [63, 127] proposed in 2013 two dual ways to obtain a continuous representation (see Figure 43) of a given signal (such as a binary or a gray-level image) on $\mathbb{H}^{n}$ : they used first the DWC

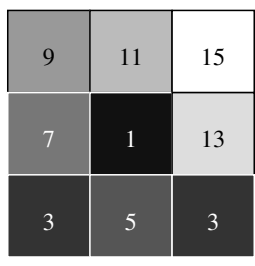

(a) $u$

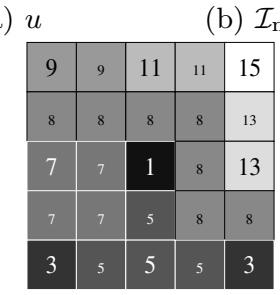

(d) $u_{\mathrm{DWC}}$

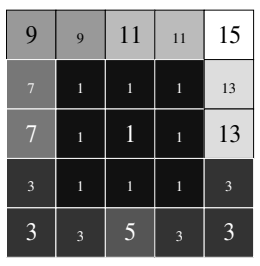

(b) $\mathcal{I}_{\min }(u)$

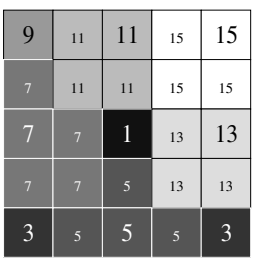

(c) $\mathcal{I}_{\max }(u)$

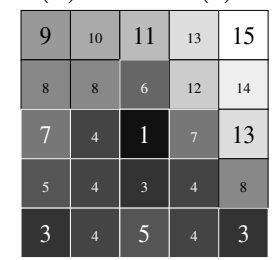

(e) $\mathcal{I}_{\text {med }}(u)$

Fig. 42: Given an integer-valued function $u$, depicted in (a), we have the dual interpolations $\mathcal{I}_{\min }(u)$ and $\mathcal{I}_{\max }(u)$ depicted in (b) and (c) respectively, and the self-dual digitally well-composed interpolation $u_{\mathrm{DWC}}$ depicted in (d); sub-figure (e) depicts a local self-dual interpolation $\mathcal{I}_{\text {med }}(u)$ based on the median operator.

min/max interpolations $u_{\min }$ and $u_{\max }$ of Mazo [117, and then they computed their span-based immersions on $\mathbb{H}^{n}$, to obtain respectively $U_{\min }$ and $U_{\max }$.

The resulting representation is then continuous as an upper semi-continuous map [8], shortly USC map, in the sense that the strict threshold sets are open and the large threshold sets are closed. Furthermore, these representations are $\mathrm{AWC}$ in $2 \mathrm{D}$ and $3 \mathrm{D}$; according to Boutry [26], they should also be AWC in $n$-D, $n \geq 4$. However, these representations are not self-dual: they overemphasize the bright or the dark components depending on the chosen interpolation.

Then, Boutry et al. proposed a self-dual DWC $n$ D self-dual interpolation called $u_{\mathrm{DWC}}$ [29, on which they applied a span-based immersion, to obtain the numerical scheme [26] presented into Figure 44. This representation, called $U_{\mathrm{AWC}}$, is continuous, DWC, AWC (assuming that the conjecture [26] that AWCness and DWCness are equivalent is true in $n$-D), and satisfies the intermediate value theorem. Also, when the closure of the domain of the representation is unicoherent $t^{1}$ the set of shapes [127] $\mathfrak{T}$ of $U_{\mathrm{AWC}}$ is a tree, that is, two components of $\mathfrak{T}$ are either nested or disjoint.

\footnotetext{
1 A topological space is said to be unicoherent iff it is connected and for any two closed connected sets such that their union equals the whole space, their intersection is also connected.
} 
$\mathrm{u}$

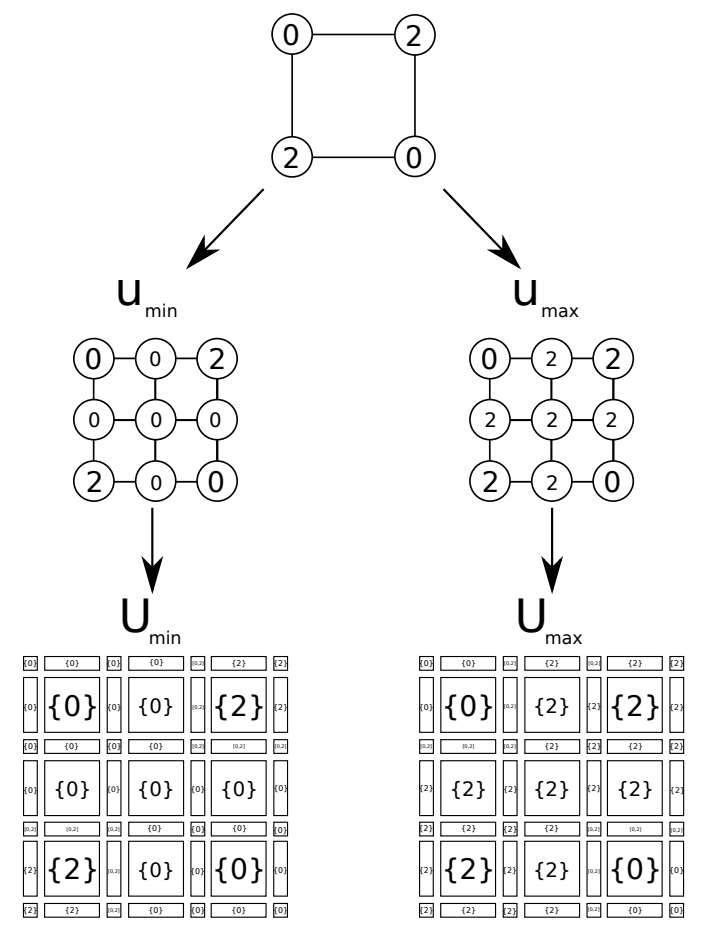

Fig. 43: Continuous dual representations $U_{\min }$ and $U_{\max }$. $\mathrm{u}$

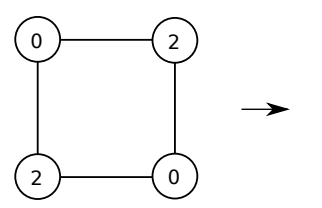

$U$

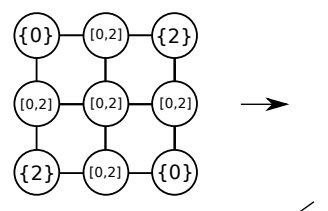

$\mathrm{u}_{\mathrm{owc}}$
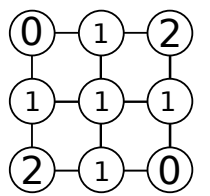

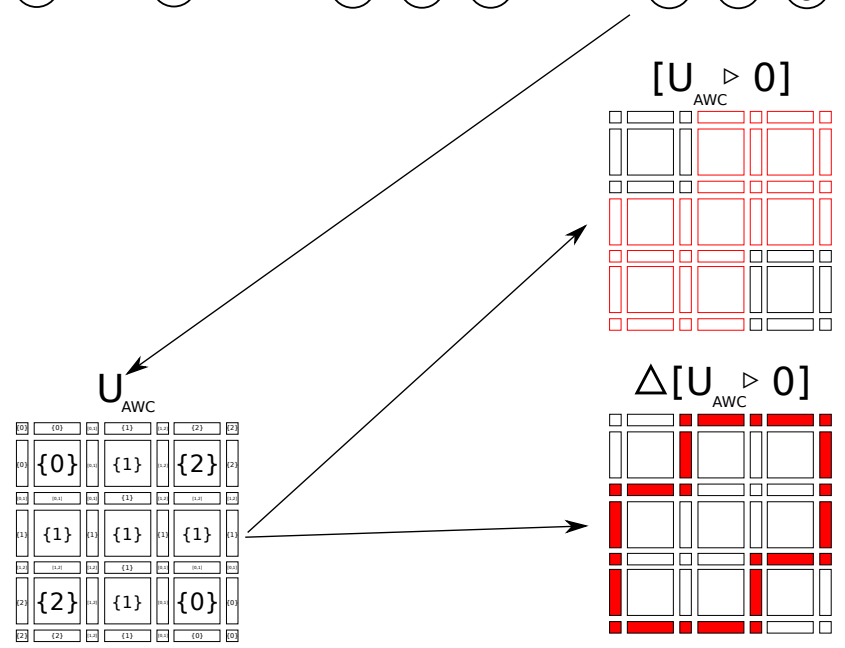

Fig. 44: Our method to obtain an AWC plain map: $\left[U_{\mathrm{AWC}} \triangleright 0\right]$ is open and its border $\triangle\left[U_{\mathrm{AWC}} \triangleright 0\right]$ is a 1-surface.

\section{Applications of well-composedness on sets}

Well-composed sets are related to many topics in digital topology: digitization of images leads generally to digital images that are not well-composed, topology of a given $2 \mathrm{D}$ signal can be preserved by rigid transformations thanks to well-composedness, topology-preserving front propagations can lead to well-composed segmentations, Euler numbers of sets can be computed locally thanks to well-composedness, well-composed Jordan curves separate the digital plane, and the boundary on the continuous analog of a well-composed set is a manifold. All these relations are developed in the section below.

\subsection{On digitization of regular images}

In image analysis, many real objects are assumed to be smooth. More exactly, they are assumed to be closed in $\mathbb{R}^{2} / \mathbb{R}^{3}$, to have a compact boundary, and such that at each point of their boundary, their tangent line/plane is well-defined [76]. This way, these subsets of $\mathbb{R}^{2} / \mathbb{R}^{3}$ are $r$-regular, that is, there exists a value $r>0$ such that, at each point of their boundary, they admit an inside (respectively, an outside) open osculating disk/ball of radius greater than or equal to $r$ lying entirely in this set (respectively, its complementary). This class of sets has been introduced in 1982 [140,158] and then used by Latecki et al. [103,101,104] and by Tajine and Ronse [175].

Then, by digitization, some topological properties may be preserved depending on the chosen digitalization (as the subset digitization [101, the Gauss digitization, the intersection digitization, the threshold-based digitization, and so on). This also depends on the chosen reconstruction method following the digitization process used to reproduce the shape of the original object as good as possible thanks to continuous analogs like Voronoï cells, cubes, or balls (centered at the voxels of the digitization and tessellating $\mathbb{R}^{n}$ ).

Then, the real object and its reconstruction can be homeomorphic (in the sense of the topological equivalence of Pavlidis [140]), or homotopy equivalent, or they can have the same homotopy tree, they can bee strongly $r$-similar (that is their morphing distance [169] is lower than or equal to $r$ ), and so on.

Since a set $X \subset \mathbb{Z}^{n}, n \in\{2,3\}$, is said to be CWC iff its reconstruction using unitary centered cubic voxels has a boundary which is made of a 1-manifold in the $2 \mathrm{D}$ case, and is made of a 2-manifold in the $3 \mathrm{D}$ case, manifoldness of the boundary of a real-object is preserved 

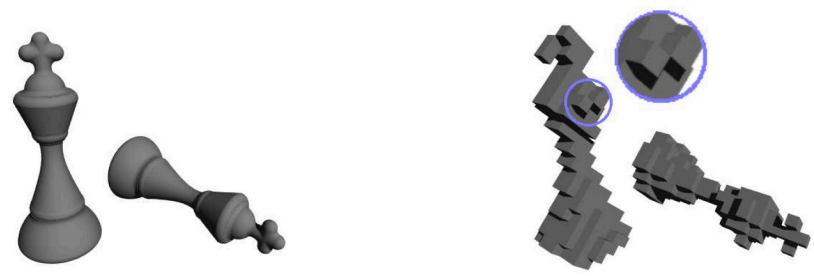

Fig. 45: A reconstruction based on cubical grids in 3D leads to critical configurations [171.

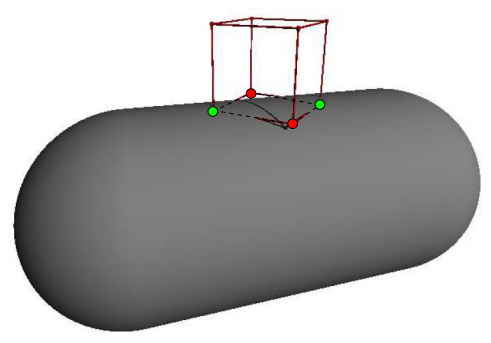

Fig. 46: Even the digitization of a smooth bordered 3manifold can contain a 2D critical configuration [171]: the two red points at the bottom of the cube lie outside the object when the two green points at the bottom of the cube lie inside the object, which leads to a $2 \mathrm{D}$ critical configuration by digitization.

iff its digitization is well-composed, assuming that we used unitary centered cubes for the reconstruction step.

Let be an object in $\mathbb{R}^{2}$. Now let us assume that $\mathbb{R}^{2}$ is tessellated with squares of diameter $r>0$ such that the barycenters of the squared pixels whose interior intersect this object are set at 1 and the barycenters of the other pixels are set at 0 . This procedure is called $2 D$ digitization by intersection, and its digitization step is equal to $r$. According to Gross and Latecki [76], digitizations by intersection of $r$-regular objects are wellcomposed sets when using a digitization step lower than or equal to $r$. This way, manifoldness of the object is preserved using this digitization.

In 3D, it has been shown by Stelldinger et al. in [171, 169 that using cubical grids, whatever the regularity of the initial object and the digitization step, we cannot ensure that the reconstructed object is well-composed (see Figure 45). Effectively, even digitizations of very regular objects can contain critical configurations, which result in pinches in the reconstruction using cubic centered voxels (see Figure 46). The same reasoning can be extended to greater dimensions.

As $r$-regularity is a very strong constraint, we could imagine that some other kinds of geometric or topolog-

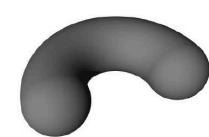

(a)

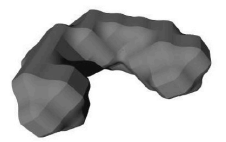

(d)

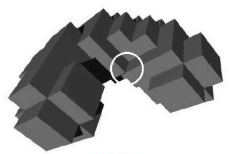

(b)

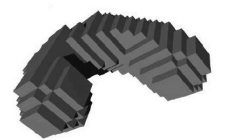

(e)

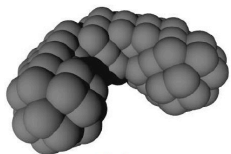

(c)

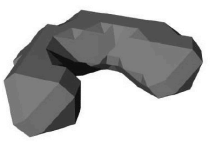

(f)
Fig. 47: A $r$-regular object and its reconstructions [171]: (a) the $r$-regular object, its reconstruction using (b) a cubic $\frac{r}{2}$-grid, (c) ball union, $(d)$ trilinear interpolation, (e) Majority Interpolation, $(f)$ MMC (Modified Marching Cubes).

ical constraints could allow to obtain well-composedness; however, it has been shown than $r$-regularity is a very good assumption to model real objects, since it is a necessary and sufficient condition for many topology preserving theorems [169].

The only possibilities seem then to be to change the grid where the digitization is realized (1), or the digitization itself (2), or the reconstruction procedure (3). In the first case, we can refer to the works of Stelldinger and Strand [172] which show that any digitization on a body-centered-cubic (BCC) or face-centered-cubic (FCC) grid ensures topology preservation if the digitization is dense enough, and then that the boundary of the reconstruction is a 2-manifold. In the second case, only the $2 \mathrm{D}$ digitization by intersection seems promising enough to ensure well-composedness, while the other digitizations do not give any guarantees. In the third case, many efficient techniques exist and ensure that the resulting boundary is a manifold whatever the given input (see Figure 47): majority interpolation [170, ball union [171, the Marching Cubes algorithm [112] (under some constraints), the trilinear interpolation [171], the smooth surface representation [171]. Note that this list may be not exhaustive.

\subsection{Rigid transformations and preservation of well-composedness}

In the continuous world, topological properties are preserved by rigid transformations, that is, compositions of a translation and a rotation. They are much used in remote sensing [166], medical imaging [141,155], image registration [10], and image warping [57]. This is not anymore the case in the discrete world [134,135]: starting from a binary image defined on a square grid, it is 

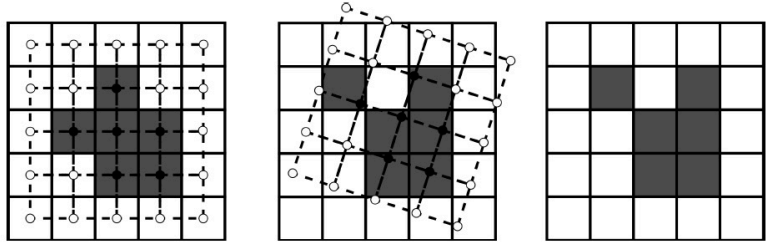

Fig. 48: An image and a possible rotation, i.e., a rigid transformation [137.

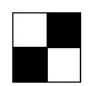

(a) $\mathscr{C}_{1}$

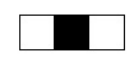

(b) $\mathscr{C}_{2}$

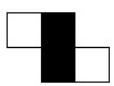

(c) $\mathscr{C}_{3}$
Fig. 49: Forbidden pattern in regular images [137].

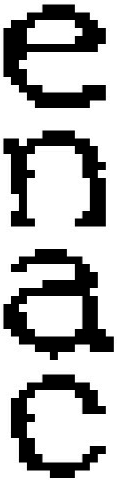

(a)
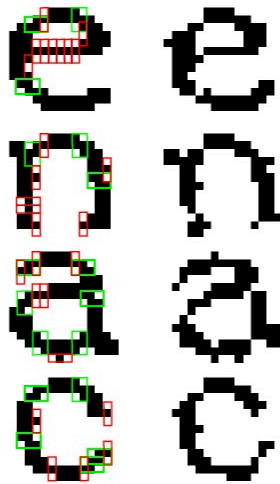

(b)

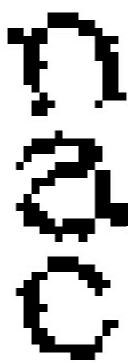

(c)
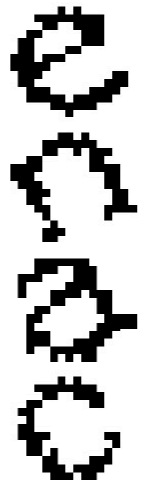

(d)
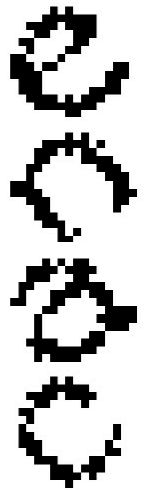

(e)
Fig. 50: Patterns that are completely destructured by the rigid transformation [138].

often mandatory to discretize the result of a continuous rigid transformation of this image since its domain must belong to $\mathbb{Z}^{2}$ (see Figure 48 .

This results in the loss of digital topological properties, especially based on connectivities, like well-composedness, or in modifications in the adjacency tree [147] (a tree-based representation of the nested relationship between the connected components in the image). This way, the two images cannot be topologically equivalent 152 .

Fortunately, Ngo et al. [137, 138, 136] studied under which conditions the topology of a $2 \mathrm{D}$ digital image is preserved under discrete rigid transformation (DRT) and proved that if the initial image is regular (a criterion based on some forbidden patterns described in Figure 49 including the usual critical configurations of Latecki [104]), then the resulting DRT is well-composed and the adjacency trees of the initial and final im-

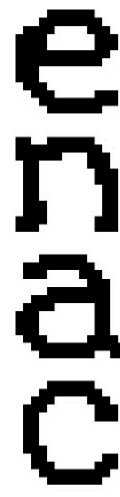

(a)
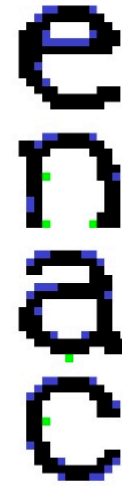

(b)

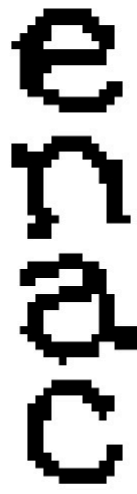

(c)

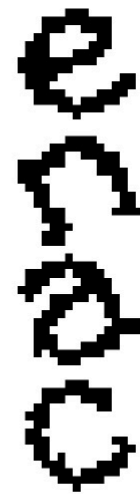

(d)

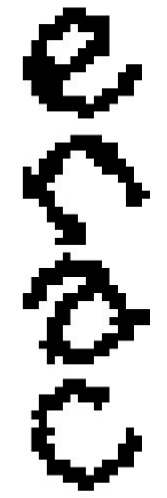

(e)
Fig. 51: Modified patterns whose topology is preserved under the rigid transformation [138].

ages are isomorphic. In that sense, they are "topologically equivalent". Making regular any image is then straightforward, using for example a super-resolution strategy [137] able to make any well-composed image regular. Figure 50 shows letters whose topology is lost under rigid transformation due to the local critical patterns depicted in red: 4-connected components are decomposed into several other 4-connected components, and the 8-components corresponding to the holes are merged with the background. Figure 51 shows the same letters, modified such that no critical pattern occurs and such that the rigid transformation preserves wellcomposedness and the adjacency tree. To date, no result about the preservation of well-composedness using $3 \mathrm{D}$ rigid transformations has been published.

8.3 Front propagation and well-composed segmentations

Among the family of topology constrained front propagation methods [35, 5, 174, 105, 78, 157], the works in [78 and in [157] rely on simple points [13,90,14, that is, points such that their addition or removal to the component will not change the topology of the image. They start from initial seeds distributed in the areas of interest in the space of the image, and then modify these components by adding or removing simple points. It can also be interesting to use multisimple points [157, that is points such that their addition or removal do not create or delete handles in the image.

Tustison proposed in [178 a new method based on two simultaneous criteria: topology and well-composedness 104,100,101 preservations of the seeds. This procedure is based on the identification using topological numbers [19 of points, which preserve the well- 

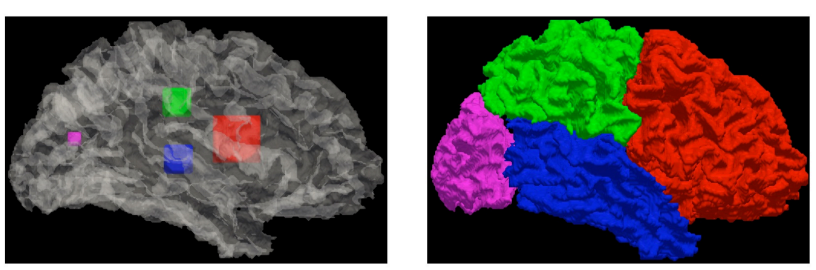

Fig. 52: From seeds to well-composed regions [178.
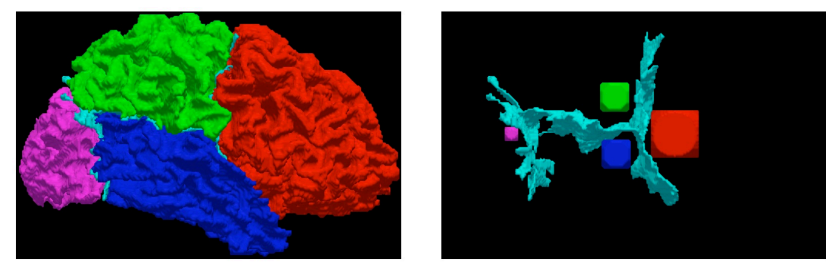

Fig. 53: Glamorous glue [178] applied to regions results in a well-composed region.

composedness and the topology of the image: these topological well-composed points are then the only points allowed to be added to the front to make it evolve. This results in a set of connected components and in an interface which are well-composed (see Figure 52): the adjacency relations are then $(4,4)$ in $2 \mathrm{D}$ and $(6,6)$ in $3 \mathrm{D}$.

Since the interface between two near components will satisfy the Digital Jordan Separation Theorem [132, 101 (DJST) thanks to their well-composedness, these components can be iteratively glued together by adding elegantly the part of the Jordan surface separating them to constitute a final segmentation which is well-composed (see Figure 53). Then, they can be visualized using the MC algorithm [112]: the use of the CCMC (ConnectivityConsistent Marching Cubes) algorithm [77] generally used to resolve the ambiguous cases is not required anymore.

8.4 Locally computable Euler characteristic thanks to well-composedness

The Euler number [90] or genus is a topological invariant used in many applications [113,184]: computer graphics, image analysis, object counting, visual inspection [4, 192, license plates characters and numbers recognition tasks [1], and real-time thresholding [164.

A subset $X$ of the plane or of the $3 \mathrm{D}$ space is said to be simplicial iff it is expressible as a finite union $C$ of vertices (0-faces), edges (1-faces), triangles (2-faces), and tetrahedra (3-faces). We also say that $C$ is the simplicial decomposition of $X$. The Euler number of this

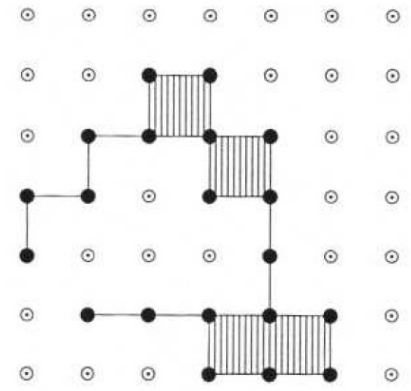

Fig. 54: Simplicial set of a $(4,8)$ digital picture whose Euler characteristic is equal to $1([90])$.

simplicial complex is then defined by the following axioms:

$-\xi(\emptyset)=0$

$-\xi(S)=1$ if $S$ is non-empty and convex,

- for any simplicial sets $S^{1}, S^{2}$,

$$
\xi\left(S^{1} \cup S^{2}\right)=\xi\left(S^{1}\right)+\xi\left(S^{2}\right)-\xi\left(S^{1} \cap S^{2}\right),
$$

and does not depend on the triangulation $C$ of $X$.

According to [176, 90], the (face) Euler number of the simplicial set $S$ can be formulated such that:

$$
\xi(S)=n_{0}-n_{1}+n_{2}-n_{3},
$$

where $n_{k}, k \in \llbracket 0,3 \rrbracket$, denotes the number of $k$-faces in the simplicial decomposition. Note that the value of the face Euler number depends on the chosen connectivity [176].

By the Euler-Poincaré formula, we obtain the formula of the volume Euler number:

$$
\varepsilon=b_{0}-b_{1}+b_{2},
$$

where $b_{k}$ is the $k$-dimensional Betti number. In fact, $b_{0}$ equals the number of connected components of the object, $b_{1}$ equals the number of holes in all these components, and $b_{2}$ equals the number of cavities in all these components. For a given binary image $F$, the sum of the volume Euler numbers of all connected components in $F$ is called the volume Euler number of the image $F$.

In the case of a planar simplicial set, the Euler number is equal to the number of connected components minus the number of holes, which permits to define easily the Euler characteristic of a 2D image where the continuous analog of the ones is represented by its corresponding planar simplicial set, which is always possible on a rectangular grid for a digital set; this point is detailed hereafter.

Assume that any 2D binary digital $(m, n)$-image $P$, where $(m, n)$ belongs to $\{(4,8),(8,4)\}$, is given, and 


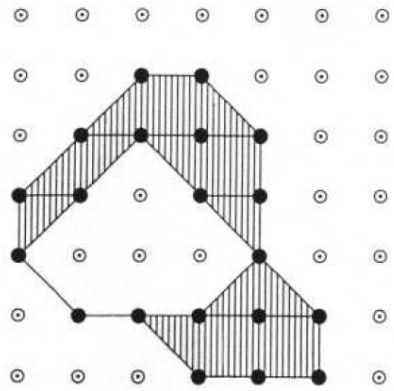

Fig. 55: Simplicial set of a $(8,4)$ digital picture whose Euler characteristic is equal to $0([90])$.

that we define, as in $90, C_{0}$ as the black point set in the image, $C_{1}$ as the union of the black segments whose endpoints are $m$-adjacent black points. If $(m, n)=(4,8)$ (respectively, if $(m, n)=(8,4))$, we define $C_{2}$ as the union of the unit squares (respectively, the $(1,1, \sqrt{2}$ ) triangles) whose sides are contained in $C_{1}$. Then we obtain $C(P)=C_{0} \cup C_{1} \cup C_{2}$, that is, the simplicial set of the image $P$. The Euler number of $P$ is then obtained by computing the number of connected components of $C(P)$ minus the number of holes in $C(P)$.

Figure 54 and Figure 55 depict two binary images with the same set of points. Figure 54 depicts an image whose Euler number is equal to one, when Figure 55 depicts an image whose Euler number is equal to zero. Effectively, the Euler number depends on the chosen connectivity. For this reason, if the given digital picture is well-composed, the choice of the adjacencies does not import, and the Euler number is unique.

Furthermore, it has been observed that using dual adjacencies on arbitrary binary digital images, this number can be computed locally [139,91] by an enumeration of some local patterns (see also [74,167] for different approaches). Since using any pair of dual adjacencies on a well-composed image leads to the same result, Latecki deduced in 104 that the Euler number is also locally computable on well-composed sets. This results in much faster algorithms, which shows one more time the powerfulness of well-composedness.

The $3 \mathrm{D}$ case is obviously also important and has been treated in [36, 48, 37, 181, 51,22, 106]. In particular, in [107, the used method is local.

Benefits of well-composedness come mainly from the fact that the number of possible configurations is minimal, and then the calculus is simplified and computations are faster.

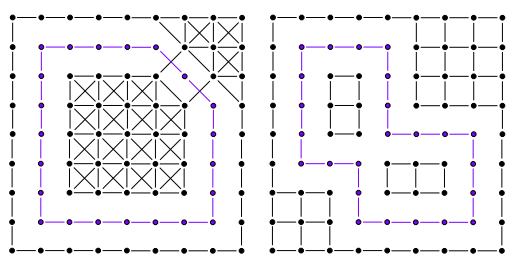

Fig. 56: 8-curves and 4-curves are not always Jordan curves in $\mathbb{Z}^{2}$.

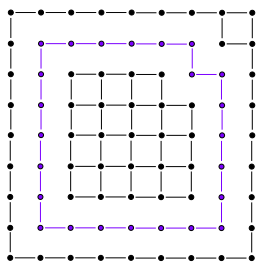

Fig. 57: A well-composed curve is always a Jordan curve in $\mathbb{Z}^{2}$.

8.5 Well-composed Jordan curves separate the plane

It is well-known that the JST 133 exposed and discussed before does not hold, for instance, in the discrete world of rectangular grids.

For example, a simple closed curve based on digital connectivity [74,82,124,125, 147, 149, 150, 151, 194, does not always separate the space into two components anymore: Figure 56 shows on the left a curve based on 8-connectivity and on the right a curve based on 4connectivity; none of them separates the digital plane $\mathbb{Z}^{2}$.

This is related to the connectivity paradoxes of Rosenfeld [153] that we exposed in Subsection 2.2 This paradox can appears when we choose the same connectivity in $\mathbb{Z}^{2}$ for a set and its complement. To obviate this problem, we can use dual pair of connectivities, and then we obtain the Digital Jordan Separation Theorem (DJST) [173, 148, 146, 145] which states that a digital 4-connected simple closed curve (whose each point has two 4-neighbors in the curve) separates the plane into two 8-connected components. Conversely a digital 8-connected simple closed curve (whose each point has two 8-neighbors in the curve) separates the plane into two 4-components.

Another way to obviate the connectivity paradox is to use well-composed simple closed curves, for which 4connectivity and 8-connectivity are equivalent: in this manner, no ambiguity is possible and the connectivity paradox cannot occur anymore. Figure 57 shows an example of well-composed simple closed curve, which is then a Jordan curve in the sense that it separates the digital plane into two components. 


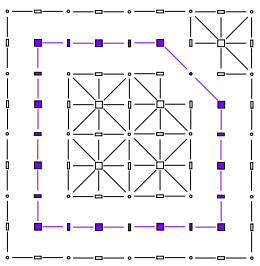

Fig. 58: A simple closed curve in $\mathbb{H}^{2}$ is a Jordan curve.
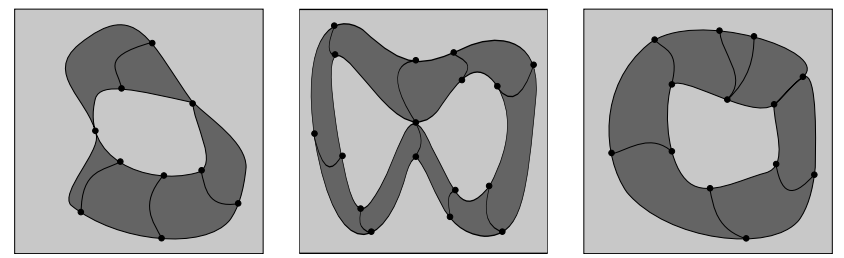

Fig. 59: Different kinds of simple closed curves according to Wang and Battacharya.

Note that another way to preserve the separation property proper to the plane is to work in Khalimsky Grids 86, 87,88] (see Figure 58, where simple closed curves, also called discrete 1-surfaces [92,55], separate the Khalimsky grid $\mathbb{H}^{2}$. However, in this case, the neighborhood of a point in $\mathbb{H}^{2}$ depends on its coordinates, and then the grid structure of $\mathbb{H}^{2}$ is different from the usual ones of $\mathbb{Z}^{2}$.

On arbitrary grids, Wang and Battacharya [183. proposed an interesting generalization of the DJST. Let $S$ be a set of pixels. Two pixels of $S$ are called edgeconnected (respectively, point-connected) in $S$ if there is a path between them such that the successive pixels on the path belong to $S$ and are directly edge-connected (respectively, directly point-connected). Two pixels of $S$ are called mix-connected in S if there is a path between them such that the successive pixels on the path belong to $\mathrm{S}$ and are either directly edge-connected or directly point-connected; they call such paths an edgeconnected, point-connected, or mix-connected path in $S$, respectively. This way, their equivalent of the Jordan Separation Theorem on arbitrary grids is the following: a finite edge-connected (resp. a finite mix-connected) simple closed curve of pixels separates the plane into two mix-connected (respectively, edge-connected) components. Furthermore, a set of pixels $S$ is said well-composed iff for any point $v$ in the boundary of $S, S \cap \mathcal{A}(v)$, where $\mathcal{A}(v)$ denotes the set of pixels which contain the point $v$, is edge-connected. Then, a well-composed simple closed curve of pixels in the sense of [183] separates $\mathbb{R}^{2}$ into two components. Figure 59 shows on the left a mix-connected simple closed curve, separating the plane into two edge-connected parts, the curve in the middle is an edge-connected simple closed curve separating the

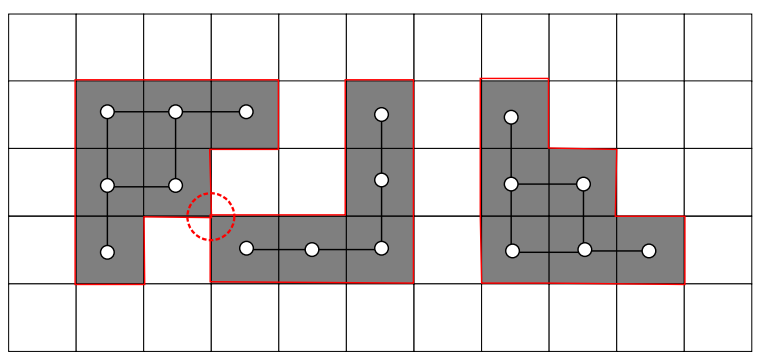

Fig. 60: The continuous analog of a set which is not wellcomposed: the topological boundary contains a "pinch".

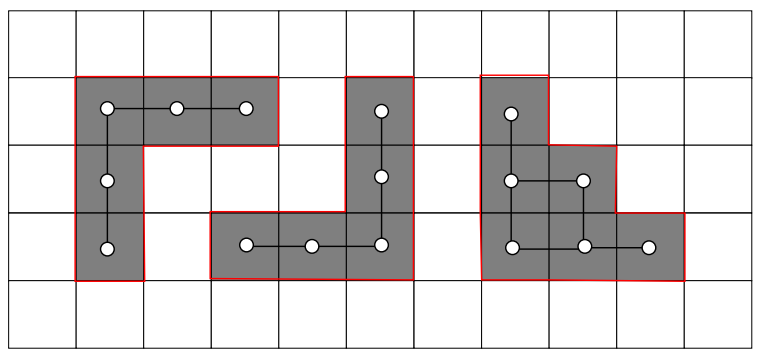

Fig. 61: The continuous analog of a well-composed set: the topological boundary is a manifold.

plane into two mix-connected components, and on the right, we can see a well-composed simple closed curve which separates the plane into two components, whatever the chosen connectivity. Well-composedness is then used here to give back to the JST the "natural" topological property verified in the continuous plane.

8.6 Jordan separation theorem and well-composedness

Well-composedness is deeply related to Jordan curves, satisfying the JST $[83,12,179,23$, and to Jordan surfaces, satisfying the Jordan-Brouwer Separation Theorem 3, 75, 110,93.

Effectively, as stated in [102, it is equivalent to say that a digital subset $X$ of $\mathbb{Z}^{2}$ is well-composed or to say that the boundary of its continuous analog is a 1manifold, which means that it is made of disjoint simple closed curves. Figure 60 shows a set which is not wellcomposed, since one of the connected components of its boundary is not a simple closed curve, and Figure 61 shows a well-composed set, since each connected component of its boundary is a simple closed curve.

Note that the fact that a simple closed curve is wellcomposed in the digital plane is different from the fact that the boundary of a $2 \mathrm{D}$ set is a simple closed curve in $\mathbb{R}^{2}$. The first concept is a property of well-composed curves as a set (in this case, the whole set is a Jordan curve), when the second correspond to the separation 
property of any boundary of any 2D well-composed set (in this case, the Jordan curves are the connected components of the boundary).

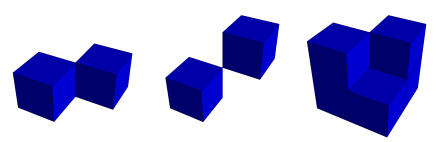

Fig. 62: Non-Jordan surfaces.

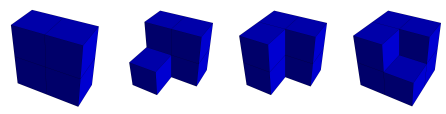

Fig. 63: Jordan surfaces.

Also, as we have seen before, a digital set $X \subset \mathbb{Z}^{3}$ is said CWC iff the boundary of its continuous analog is a 2-manifold, that is, is made of disjoint simple closed surfaces, which strongly relates the Jordan-Brouwer separation theorem to well-composedness. Figure 62 shows the boundaries of continuous analogs, which are not Jordan surfaces, and Figure 63 shows on the contrary boundaries of continuous analogs of well-composed sets, which are then 2-manifolds.

\section{Applications of well-composedness on gray-level images}

Here we present some applications related to gray-level well-composed images: we first recall that the surface extraction algorithm called Marching Cubes has no ambiguous cases, and then no hole problem. On these images, we recall how to obtain thin topological maps of digital gray-level images to compute watersheds starting from a well-composed gradient image. We also recall the different applications that exist nowadays, based on the computation of the tree of shapes on these well-composed images.

\subsection{Marching Cubes}

The main reference in matter of scientific and experimental visualization of scalar field data on $3 \mathrm{D}$ cubical grids is the Marching Cubes (MC) algorithm [112]. Assuming that we have a continuous scalar field $f$ whose values are known on the lattice points of a cubical grid, we can visualize the approximation of the implicit surface $[f=\alpha]$ (usually assumed to be a topological 2manifold), $\alpha \in \mathbb{R}$, using a triangular mesh, that is, a
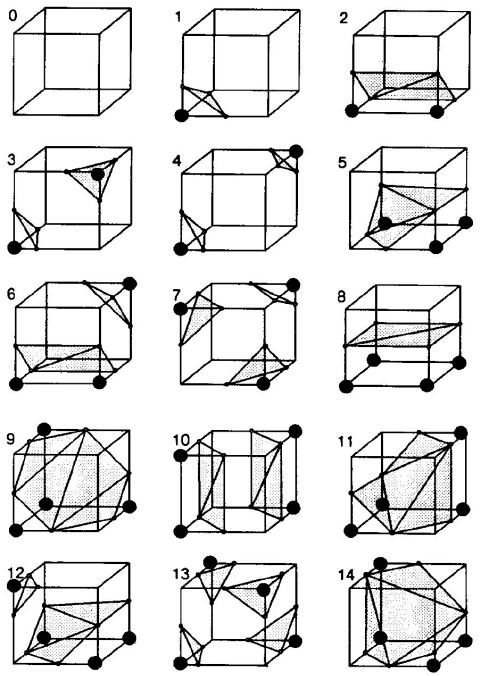

Fig. 64: Lorensen's Marching Cubes Lookup Table 112 .

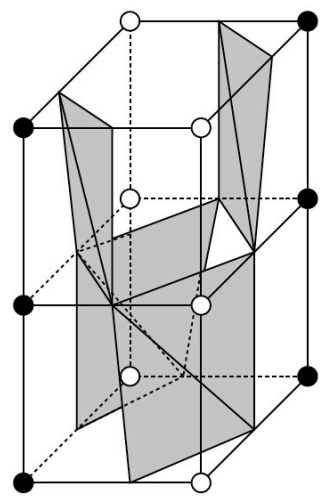

Fig. 65: The "hole problem" 162 using Marching Cubes.

simplicial complex, also called the surface tilling of the iso-surface. This algorithm computes the triangulation cube by cube in this way: each corner $c$ of the cube (the lattice points of the cubical grid) whose value $f(c)$ is greater than or equal to the given threshold $\alpha$ is said to be positive (they correspond to the inside part of the object), and the other corners of the cubes are said to be negative (they correspond to the outside part of the object). A boundary point is then created on each edge of the cube using a (trivial or non-trivial) interpolation such that one of its vertices is positive and the other is negative. Then, using a lookup table proper to the MC algorithm 112 as shown in Figure 64 boundary points are connected with one or several triangles, making a triangular mesh, connected or not, depending on the configuration of points in the cube. Then the "local" 
meshes are grouped together to make the final mesh in $\mathbb{R}^{3}$.

We would then hope that the resulting mesh is an union of disjoint simplicial surfaces [24] which separates the positive vertices to the negative vertices. However, some holes/cracks can appear as shown in Figure 65, due to ambiguities in some configurations. In this case, the algorithm fails to produce a piecewise linear manifold: some edges are faces of only one triangle, which means that they draw together the boundary of a hole in the surface. To obviate these ambiguities, Han et al [77] use digital topology: a pair of connectivities (among the 8-,18-, and 26-connectivities) is then associated with the positive/negative lattice points, to be able to decide which surface tilling has to be drawn (at each cube separatly). As usual, this pair must be a Jordan pair to avoid the connectivity paradoxes of Rosenfeld. Then there is only one possible tilling at each cube and positive and negative vertices are separated in each cube by the local tilling surface. The resulting mesh is "digitally" topologically correct in the sense that the surface tilling correctly reflects the topology of the initial isosurface $[f=\alpha]$ if the connectedness is well chosen. This algorithm is called connectivity-consistent marching cubes (CCMC).

Be careful not to confuse topological correctness used in digital topology and topological correctness used in isosurface extraction: this last means that the approximating isosurface is homeomorphic to piecewise trilinear interpolation of the digitization of the given continuous scalar field.

However, it is sometimes difficult to choose which connectivity is best suited to a given application, and then we would rather avoid to choose a connectivity, since the resulting mesh depends strongly on this choice. Digital well-composedness is then salutary: it has been stated in 89 that a cube is unambiguous iff there exists a 6-path of positive (respectively, negative) vertices in this cube connecting each pair of positive (respectively, negative) vertices of this same cube, which is the characterization of DWCness in 3D. This way, no choice of connectivity is needed anymore, since whatever the chosen connectivities the result will be the same. Moreover, $[f=\alpha]$ is a piecewise linear 2-manifold with no hole, and then its boundary is contained in the boundary of the cubical grid; this last property is called topological consistency.

Furthermore, Siqueira et al. proved in 162 that the isosurface resulting from the MC algorithm may reflect the topology of the initial continuous scalar field when the given binary image is well-composed. If for some

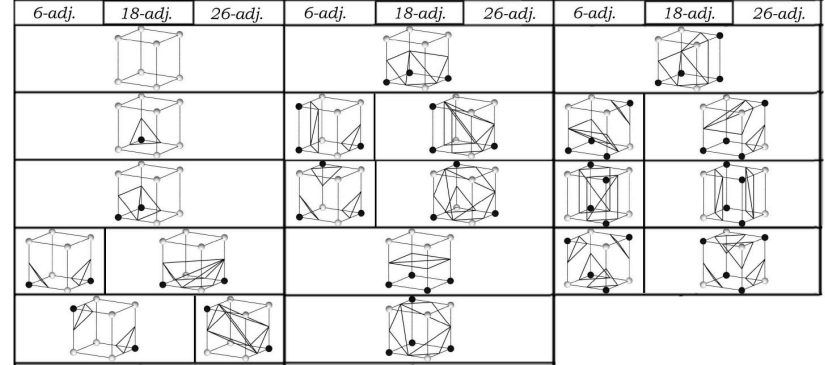

Fig. 66: Lookup tables of Daragon 44,45, in the 3D case.

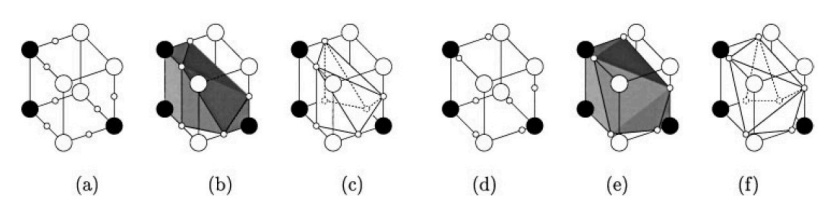

Fig. 67: $n$-D approach of Lachaud [98, based on the convex hull.

reason, we are not able to make any well-composed interpolation or to use any topological repairing method, the use of the Modified Marching Cubes algorithm [170, shortly MMC, is a good choice, but it assumes that the digitized object is $r$-regular and that the sampling grid has a sufficient resolution.

Note that some very powerful MC-like methods exist for the $n$-dimensional case, $n \geq 2$, as the frontier orders of Daragon [44,45] based on combinatorial topology and the continuous analog of the digital boundary of Lachaud and Montanvert 98 based on digital topology. Both obtain the same surface tillings in the $3 \mathrm{D}$ case, as shown in Figure 66, showing each possible configuration in the $3 \mathrm{D}$ case, assuming that we use 6-,18-, or 26-connectivity for the black points (and a dual connectivity for the white points). In other words, $(6,18)$-connectivity will join the black points which are 6 -connected, and will separate the black points which are only 18 - or 26-connected. At the same time, it will join the white points which are 6- or 18-connected, and it will separate the white points which are only 26connected. Note that the $n-\mathrm{D}$ approach of Lachaud is depicted in Figure 67, and consists in separatly computing in each cube the boundary of the convex hull of the set of points made with the black points plus the boundary points (see $(a),(b)$, and $(c))$. An equivalent approach using a non trivial interpolating function is showed from $(d)$ to $(f)$. Under reasonable constraints, these two methods provide simplicial surfaces with no "holes", at least in the 3D case. 


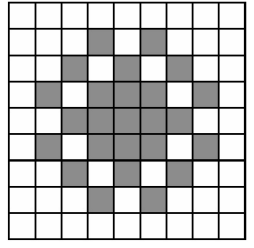

Fig. 68: The irreducible thick configuration [7].

We can also mention the existence of isosurface simplification algorithms [160,84 used to reduce the excessive number of triangles produced by the MC algorithm in practice. These methods work particularly well with 3D CWC images since they preserve the topology of the boundary of the continuous analog of the foreground of a well-composed digital image [162].

Finally, Boutry [26] exposed in his thesis that a $n$-D image which is DWC should not have any possible local ambiguity, since DWCness implies local equivalence of connectivity, and in this manner gray-level DWC images should not have ambiguous cases. Furthermore, he conjectured that no "hole problem" is possible in $n$-D using DWC images. No proof of this assertion has been published yet.

9.2 Thin topological maps thanks to well-composedness

A discrete image can be seen as the digitization of a piecewise continuous function. This way, we can represent the underlying piecewise continuous function of a discrete image using a topological map where faces correspond to the smooth regions and where the contours made of edges and vertices correspond to the discontinuities of this underlying function. Note that this representation using faces, edges and vertices is not new [59, 96. However, consistency problems, like the "irreducible thick configuration" of [7] in Figure 68 or [18, 90], are encountered when we work on cubical grids in this context: there is then no guarantee that the extracted $c r e s t$ network [115] is thin.

To obviate this problem, Marchadier et al. [115] propose to use well-composedness [104,17, 102] to avoid the connectivity problem and to obtain a coherent topological map where the resulting crest network is thin. The proposed method is the following. Starting from a given $2 \mathrm{D}$ grayscale image, they compute the gradient that they make well-composed using some topological repairing method [115. Then they apply a leveling method of Bertrand et al. [18] which combines the well-composed-preserving thinning of Latecki [104] and

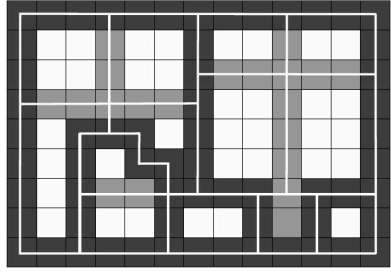

Fig. 69: An irreducible gray-level well-composed image and its crest network [115].
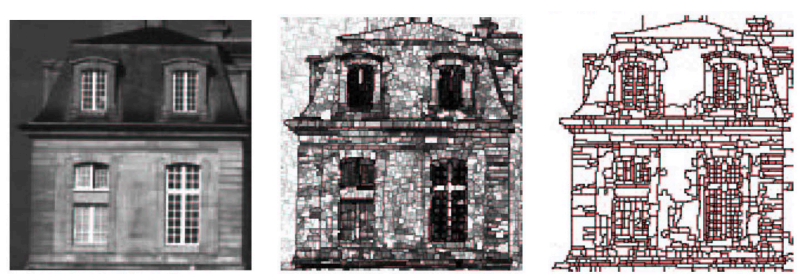

Fig. 70: The initial image, the reduced gradient, and the resulting watershed [115].
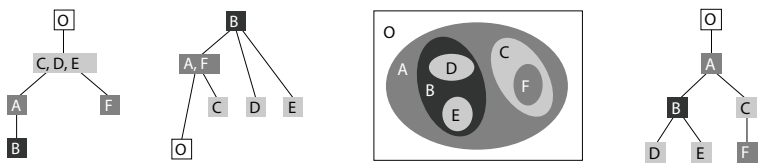

Fig. 71: A gray-level image (in the middle), its min-tree and its max-tree (on the left), and its tree of shapes [63] (on the right).

deletion of the peaks, to obtain finally an irreducible well-composed gray-level image (see Figure 69.

In fact, we can see this last image as a watershed transform [20,130,143, 126, 16] of the gradient of the initial image. Effectively, the quasi-minima of this gradient represent the catchment bassins. Then, by a caseby-case study, a thin crest network is computed on the complement of these quasi-minima, using a linking method [120,47], with no ambiguities since this image is well-composed. This way, Marchadier et al. obtain a coherent topological map [30,60] (see Figure 70 representing the underlying piecewise continuous function of the given discrete image.

\section{3 "Pure" self-duality}

A very powerful hierarchical representation, based on the inclusion relationship of the components of an image, exists in mathematical morphology: the tree of shapes [123, 63, 42] (see [119, 187, 188, 189, 186] for some possible applications). It is sometimes seen as the fusion of the min-tree, made of the connected components 


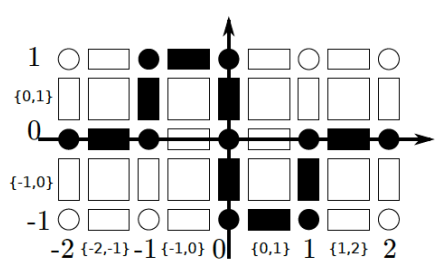

Fig. 72: An example of plain-map from $\mathbb{H}^{1}$ to $\mathbb{H}^{1}[127$.
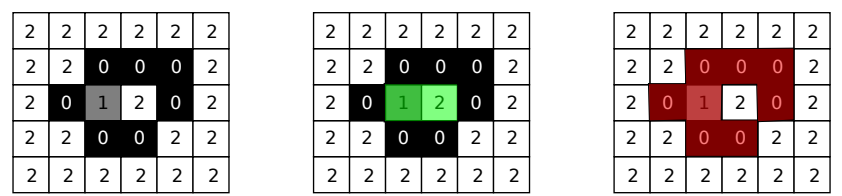

Fig. 73: Incoherences using 4-connectivity for both upper and lower threshold sets: some shapes intersect but are not nested the one in the other one.
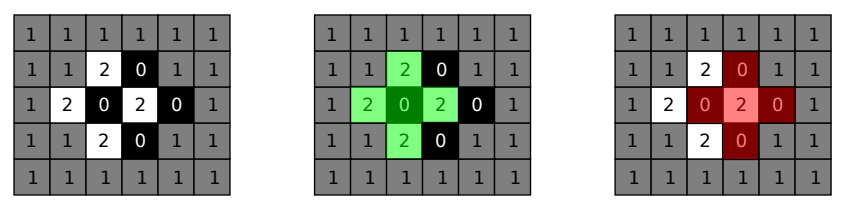

Fig. 74: Incoherences using 8-connectivity for both upper and lower threshold sets: some shapes intersect but are not nested the one in the other one.

of the lower threshold sets (leaves are the regional minima in the image), and its dual, the max-tree, which is made of the connected components of the upper threshold sets (leaves are the regional maxima in the image). Figure 71 shows a gray-level image and its component trees.

The conditions that the tree of shapes of an image exists has been developed in details in [127: summarily, if the image is a plain map (see Figure 72, that is, an interval-valued map from $\mathbb{H}^{n}$ to $\mathbb{H}^{1}$ which is closed-valued and whose domain is the whole space (assumed to be unicoherent [34]), and if this plain map is AWC, then the saturation operator (cavity-fill-in operator) and the closure operator can be "switched" (see Lemma 20 in [127]); this way, the set of quasi-shapes is a tree, i.e., two components are either nested or disjoint, and the set of shapes computed using these quasishapes is a tree too. In other words, the tree of shapes exists when computed on an AWC plain map. Note that all the details about the computational aspects are given in 63.

This morphological representation is self-dual in the sense that it is invariant by contrast inversion: it treats in a similar way bright objects over a dark background (a) $u$.

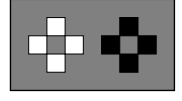

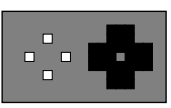

(b) $u_{\min }$.

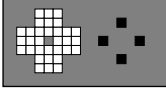

(c) $u_{\max }$.

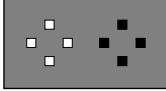

(d) $u_{\text {DWC }}$.
Fig. 75: An image $u$, its $\min / \max$ interpolations, and $u_{\text {DWC }}$ which is self-dual.

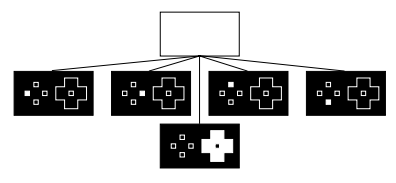

(a) $\mathcal{T}\left(u_{\min }\right)$.

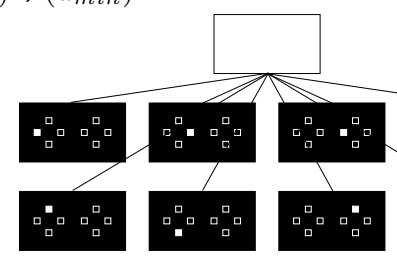

(c) $\mathcal{T}\left(u_{\mathrm{DWC}}\right)$.

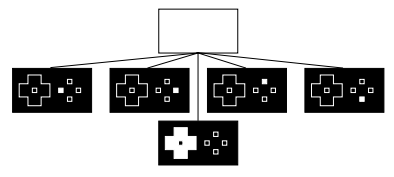

(b) $\mathcal{T}\left(u_{\max }\right)$.
Fig. 76: The tree of shapes of the min, max, and selfdual interpolations.

or dark objects over a bright background. This is very useful when we do not know a priori the contrast of the object, or if we need to study several objects of different contrasts in the same image. This operator is based on connectivities, and then, in Rosenfeld's framework, we have to associate one connectivity to the upper threshold sets and one of its dual connectivities to the lower threshold sets to avoid incoherences [9,34]: see Figures 73 and 74 By contrast, using an EWC image, connectivities are equivalent, and then we can compute the tree of shapes of an image or of its negative with the same pair of connectivities: no switch of connectivities is needed [62]; Géraud and Najman 663] call this phenomenon "pure self-duality".

Note that AWCness of the representation of the initial image is sufficient to obtain that the computed tree of shapes is effectively a tree from a theoretical point of view, when EWCness is sufficient to ensure that pure self-duality is obtained when computing the tree of shapes in practice. A self-dual DWC interpolation such as $u_{\text {DWC }}$ is then sufficient in $2 \mathrm{D} / 3 \mathrm{D}$ to ensure that we have at the same time existence of the tree of shapes and pure self-duality.

Now let us present a simple example of pure selfduality in $2 \mathrm{D}$ related to the tree of shapes. Since the front propagation in the computation of the tree of shapes [63] is based on $2 n$-connectivity, we can "emulate" the dual pair of connectivities $\left(c_{2 n}, c_{3^{n}-1}\right)$ (which connects the zeros and disconnect the ones) using a min 


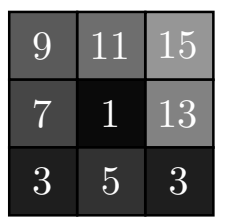

(a) $u$.

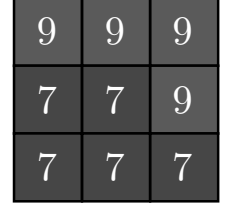

(b) $G_{c_{4}, c_{8}}(u)$

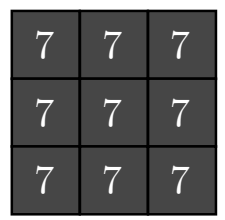

(c) $G_{c_{8}, c_{4}}(u)$.

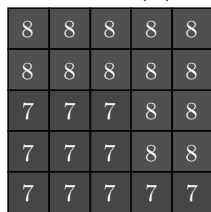

(d) $G\left(u_{\mathrm{DWC}}\right)$.

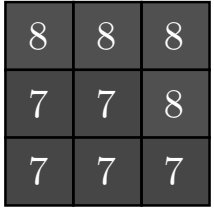

(e) Emersion of $G\left(u_{\mathrm{DWC}}\right)$
Fig. 77: Grain filterings on an image: using the DWC self-dual representation $u_{\mathrm{DWC}}$ leads to "pure" selfduality.

interpolation. In the same way, we can compute the tree of shapes based on the dual pair $\left(c_{3^{n}-1}, c_{2 n}\right)$ (which connects the ones and disconnects the zeros) using a max interpolation. So, starting from a given image $u$, we compute its min, max, and self-dual interpolations as shown in Figure 75 . Then we compute their respective trees of shapes $\mathcal{T}\left(u_{\min }\right), \mathcal{T}\left(u_{\max }\right)$, and $\mathcal{T}\left(u_{\mathrm{DWC}}\right)$ as shown in Figure 76 . We can observe that the upper and lower threshold sets are not processed in the same way using the min and max interpolations, contrary to the tree of shapes computed on $u_{\text {DWC }}$ [26] which treats exactly in the same way bright and dark components.

Note that any self-dual operator which is based on connectivities, derived [33,190] from the tree of shape or not, will be purely self-dual on well-composed images. Effectively, an example of self-dual operator derived from the tree of shapes is the grain filter, which removes the shapes in the hierarchical representation of an image $u$ whose area is smaller than a given threshold. We can remark easily in Figure 77 that using the connectivities $\left(c_{4}, c_{8}\right)$ or $\left(c_{8}, c_{4}\right)$ does not lead to the same result, when the use of a DWC self-dual interpolation such as $u_{\mathrm{DWC}}$ gives the same result whatever the chosen pair of connectivities.

Furthermore, in digital topology, since we generally assume that a Jordan pair of adjacencies is associated with a binary or gray-level image to obviate connectivity paradoxes, a specific adjacency is then considered depending on the local values of the pixels in the image; this way, we assume that there exists an "underlying structure" in the graph of the image (see Figure 78. DWC images having their connectivities locally equivalent, they can be seen as $(2 n, 2 n)$ images. This way, the underlying graph of the image becomes simpler, regu-

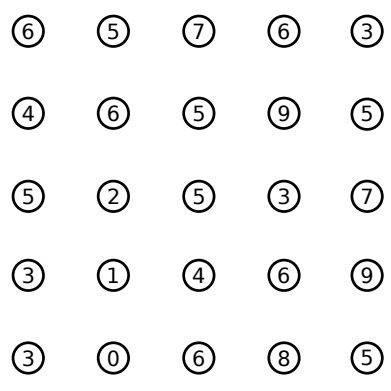

(a) $u$.

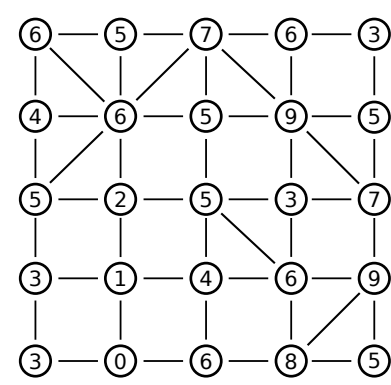

(b) Underlying graph structure.
Fig. 78: From $u$ to its underlying graph structure using the dual pair $\left(c_{8}, c_{4}\right)$ : we connect pairs of diagonal pixels whose values are greater than the two other diagonal values.

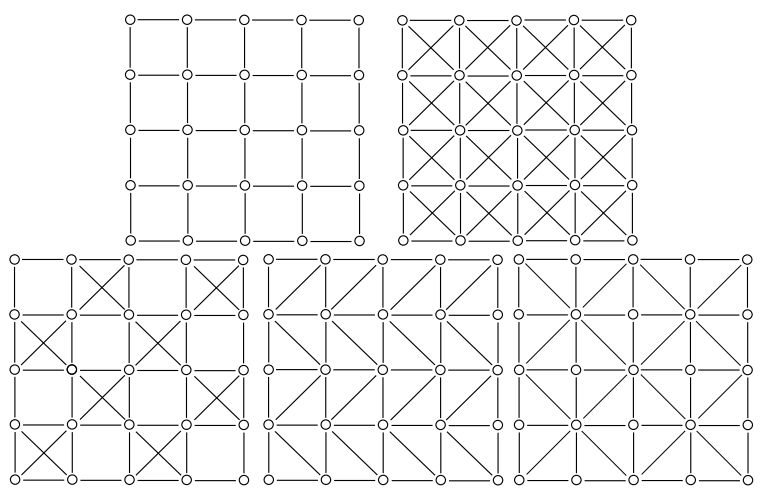

Fig. 79: All the possible cubical connectivity grids are equivalent on a digitally well-composed image: in the raster scan order, the 4-connectivity grid, the 8-connectivity grid, the perfect fusion grid, a 6 connectivity grid, and the Khalimsky grid.

lar, 90-degrees-rotation- and translation-invariant, and is not anymore correlated to the values in the image. This way, the perfect fusion grid [40,39,41], the Khalimsky grid [87], and so on, can be associated with a DWC image (see Figure 79.

\subsection{Tree of shapes of the Laplacian}

In this section, we present some results of Huyhn et al. [81] obtained thanks to the computation of the tree of the sign of the DWC morphological Laplacian in a self-dual way. Even if it is used here for text detection, this approach may be extended to treat $n$-D signals, such as M.R images, videos, or CT-scans.

Nowadays, text detection methods [56, 193, 196] are widely used, especially on mobile devices, for recognition tasks. They are generally classified into connectedcomponents-based approaches, like FASText [31], the 


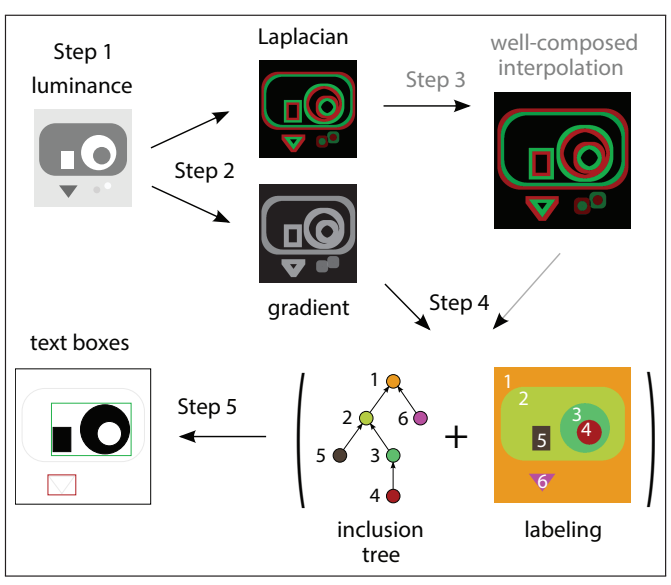

Fig. 80: Summary of the method used by Huyhn et al. 81 .

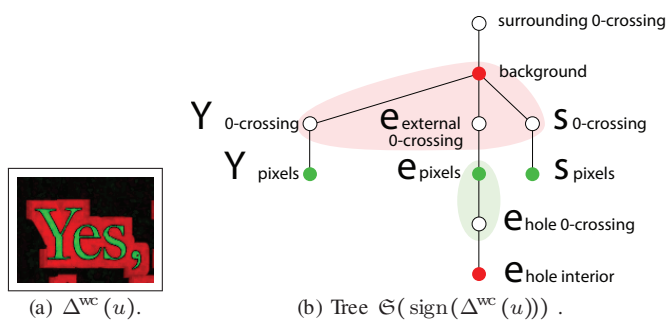

Fig. 81: An inclusion tree and its corresponding image 81 .

SWT (Stroke Width Transform) [54, the TMMS (Toggle Mapping Morphological Segmentation), and the $M$ SER (Maximally Stable Extremal Regions), or into sliding-windows approaches using $S V M$ (Support Vector Machines) [38], AdaBoost [108], or CNN (Convolutional Neural Networks) 182 as classifiers.

The one presented by Huyhn et al. [81] is part of the connected-components-based approaches, and consists in transforming an image into a tree-based hierarchical representation (see Figure 80, based on adjacency and inclusion relationship between the components in the image.

To proceed, they compute the Laplacian of a given image using a morphological Laplacian operator [180, 165, 131, whose zero-crossings are known to be very precise contour estimations of the initial image. Using a large-sized structuring element relatively to the size of the text to detect, spurious contours are easily eliminated and salient contours preserved, thanks to the non-linearity of the operator. After that, a selfdual well-composed interpolation [29] of this Laplacian is computed; this way, the zero-crossings of this interpolation are simple closed curves. Using these separated Jordan curves, we can naturally induce a hierarchy 28
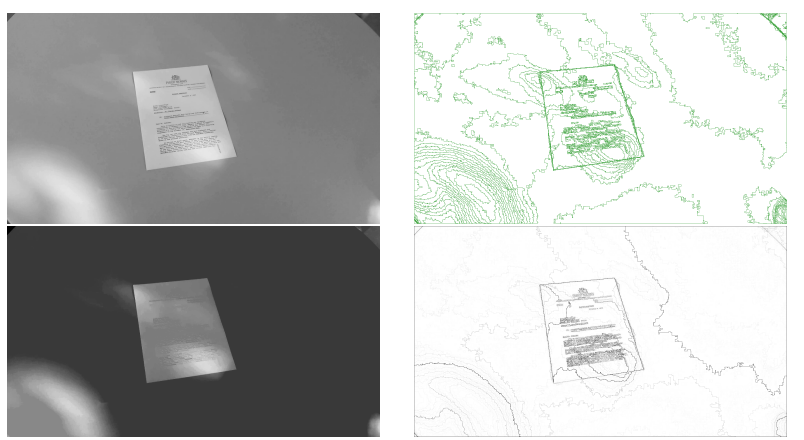

Fig. 82: In the raster scan order: the initial image, its level lines, the Dahu pseudo-distance, and its saliency map 64 .

in the image: the components given by the interior of these curves (whatever the chosen connectivity) are either nested or disjoint. A component labeling of the sign of the Laplacian and the generation of the inclusion tree are then easy and fast to compute (a classical blob labeling algorithm is sufficient).

Thanks to this tree-based representation of the image, we can extract text candidates: a hole of a character or a solid character are leaves of the tree (see Figure 81, and so on. Text grouping is then simply a subtree of this inclusion tree, since characters must be grouped iff they belong to the same background.

Finally, well-composedness gave access to a self-dual text detection algorithm which is furthermore in linear time and very efficient, thanks to the hierarchy induced by the Jordan curves extracted from the well-composed Laplacian.

\subsection{The Dahu pseudo-distance}

The minimum barrier distance, or $M B$ distance for short, is defined as the minimal interval of gray-level values along the path between two points in an image. This distance has been shown very efficient to proceed to salient object segmentation [177,[195]. A continuous version of this distance, the Dahu pseudo-distance, has then been proposed in 64]; note that this is not a distance but a pseudo-distance in the sense that it does not satisfy the identity of indiscernibles. Besides, this distance leads to topological issues in its naive version at saddle points, and for this reason, a slightly modified version of this distance has been proposed to overcome these issues: starting from a vertex-valued graph $u$, Géraud et al. compute first the self-dual interpolation $u_{\text {DWC }}$ to get rid of possible saddle 0D points (note that saddle faces still exist), and then they compute its selfdual span-based immersion into the Khalimsky grids to 
obtain a self-dual continuous representation of the initial signal. Then, no crossing of level-lines is possible, and the computation of the tree of shapes, used here to obtain the exact value of the Dahu pseudo-distance, is easy. Note that thanks to this discrete framework, all the computations remain tractable. An example of saliency map [185] is depicted in Figure 82

\section{Conclusion}

As we have seen in this survey, the connectivity paradoxes of Rosenfeld lead to ambiguities in digital images in the sense that we have to choose between dual pairs of connectivities; well-composedness is then salutary since in this context no topological paradox or ambiguity is possible. Fortunately, making an image wellcomposed is straightforward in $n$-D thanks to topological reparations and well-composed interpolations that have been developed these last years.

We have also seen that many topics of digital topology are linked to well-composedness: thinnings, segmentation, contour extraction, and visualization techniques. Furthermore, very interesting applications of well-composedness such as the Dahu pseudo-distance or the tree of shapes of the sign of the Laplacian lead to very nice results in matter of image segmentation, text extraction, and so on.

To conclude, we think that promising research directions could be to study the equivalences between the different flavours of well-composedness in $n$-D, or to study which other properties well-composed images own compared to the continuous world; we know at least that boundaries of threshold sets are Jordan curves, that binary and gray-level images have regularity properties, but it seems also that critical points such as studied in Morse theory 61] are isolated and open thanks to self-duality of this class of images [127]. The link between topological persistence [53] and the tree of shapes [34,63 could also be investigated; see for example the work of $\mathrm{Xu}$ et al. [191, which detects local invariant features in images thanks to Morse theory and the tree of shapes. On a related theme, many problems in image analysis, digital processing and shape optimization can be expressed as variational problems involving the discretization of some discrete discrete differential operators [46, 121,73] such as normal vectors or curvatures [128, 129] of digital surfaces which are of prime importance in geometry processing. This is in particular the case for the Laplace-Beltrami operator, much used in computer graphics [109,154 and in biomedical imaging [161. Recently, Caissard et al. 32. showed that particular digitizations of this operator, taking into account the discrete nature of the space (such as digital surfaces embedded in 3D), leads to better properties of convergence and precision. Hence, a question would be to understand how well-composedness helps to make such operators more "stable" from a topographical point of view.

\section{References}

1. Wisam Al Faqheri and Syamsiah Mashohor. A realtime Malaysian automatic license plate recognition (MALPR) using hybrid fuzzy. IJCSNS International Journal of Computer Science and Network Security, 9(2):333-340, 2009.

2. Pavel S Aleksandrov. Combinatorial topology, volume 1. Courier Corporation, 1956.

3. James W Alexander. A proof and extension of the JordanBrouwer separation theorem. Transactions of the American Mathematical Society, 23(4):333-349, 1922.

4. Paul Alexandroff and Heinz Hopf. Topologie I: Erster Band. Grundbegriffe der Mengentheoretischen, Topologie ,Topologie der Komplexe. Topologische Invarianzsätze und Anschliessende Begriffsbildungen. Verschlingungen im nDimensionalen Euklidischen Raum Stetige Abbildungen von Polyedern. Springer-Verlag, 2013.

5. Oleg Alexandrov and Fadil Santosa. A topologypreserving level set method for shape optimization. Journal of Computational Physics, 204(1):121-130, 2005.

6. Pavel SergueÃrevitch Alexandrov. Diskrete RÃdume. Matematicheskij Sbornik, 2(44):501-519, 1937.

7. Carlo Arcelli. Pattern thinning by contour tracing. Computer Graphics and Image Processing, 17(2):130-144, 1981.

8. Jean-Pierre Aubin and Hélène Frankowska. Set-valued analysis. Springer Science \& Business Media, 2009.

9. Coloma Ballester, Vicent Caselles, and Pascal Monasse. The tree of shapes of an image. ESAIM: Control, Optimisation and Calculus of Variations, 9:1-18, 2003.

10. Pierre-Louis Bazin, Lotta Maria Ellingsen, and Dzung L Pham. Digital homeomorphisms in deformable registration. In International Conference on Information Processing in Medical Imaging, pages 211-222. Springer, 2007.

11. Pierre-Louis Bazin and Dzung L Pham. Topologypreserving tissue classification of magnetic resonance brain images. IEEE transactions on medical imaging, 26(4):487-496, 2007.

12. Gordon O Berg, William H Julian, Ray Mines, and Fred Richman. The constructive Jordan curve theorem. Rocky Mountain Journal of Mathematics, 5(2):225-236, 061975.

13. Gilles Bertrand. Simple points, topological numbers and geodesic neighborhoods in cubic grids. Pattern Recognition Letters, 15(10):1003-1011, 1994.

14. Gilles Bertrand. A Boolean characterization of threedimensional simple points. Pattern Recognition Letters, 17(2):115-124, 1996.

15. Gilles Bertrand. New notions for discrete topology. In Discrete Geometry for Computer Imagery, volume 1568 of Lecture Notes in Computer Science, pages 218-228. Springer, 1999.

16. Gilles Bertrand. On topological watersheds. Journal of Mathematical Imaging and Vision, 22(2-3):217-230, 2005.

17. Gilles Bertrand, Jean-Christophe Everat, and Michel Couprie. Topological approach to image segmentation. In SPIE's 1996 International Symposium on Optical Science, 
Engineering, and Instrumentation, pages 65-76. International Society for Optics and Photonics, 1996.

18. Gilles Bertrand, Jean-Christophe Everat, and Michel Couprie. Image segmentation through operators based on topology. Journal of Electronic Imaging, 6(4):395-405, 1997.

19. Gilles Bertrand and Grégoire Malandain. A new characterization of three-dimensional simple points. Pattern Recognition Letters, 15(2):169-175, 1994.

20. Serge Beucher and Christian Lantuéjoul. Use of watersheds in contour detection. International Workshop on Image Processing: Real-time Edge and Motion Detection/Estimation, 1979.

21. Serge Beucher and Fernand Meyer. The morphological approach to segmentation: The watershed transformation. Optical Engineering, New York, Marcel Dekker Incorporated, 34:433-433, 1992.

22. Hanspeter Bieri and Walter Nef. Algorithms for the Euler characteristic and related additive functionals of digital objects. Computer vision, graphics, and image processing, 28(2):166-175, 1984.

23. Errett Bishop and Douglas S Bridges. Constructive analysis, volume 279. Springer Science \& Business Media, 2012.

24. Ethan D Bloch. A first course in geometric topology and differential geometry. Springer Science \& Business Media, 1997.

25. Isabelle Bloch, Henk Heijmans, and Christian Ronse. Mathematical morphology. In M. Aiello, I. PrattHartmann, and J. Van Benthem, editors, Handbook of Spatial Logics, pages 857-944. Springer, 2007.

26. Nicolas Boutry. A study of well-composedness in $n-D$. PhD thesis, Université Paris-Est, France, 2016.

27. Nicolas Boutry, Thierry Géraud, and Laurent Najman. On making $n \mathrm{D}$ images well-composed by a self-dual local interpolation. In E. Barcucci, A. Frosini, and S. Rinaldi, editors, Discrete Geometry for Computer Imagery - Proceedings of the 18th International Conference on Discrete Geometry for Computer Imagery (DGCI), volume 8668 of Lecture Notes in Computer Science, pages 320-331, Siena, Italy, September 2014. Springer.

28. Nicolas Boutry, Thierry Géraud, and Laurent Najman. How to make images well-composed in $n \mathrm{D}$ without interpolation. In Proceedings of the 22nd IEEE International Conference on Image Processing (ICIP), pages 2149-2153, Québec City, Canada, September 2015.

29. Nicolas Boutry, Thierry Géraud, and Laurent Najman. How to make $n$-D functions digitally well-composed in a self-dual way. In J.A. Benediktsson, J. Chanussot, L. Najman, and H. Talbot, editors, Mathematical Morphology and Its Application to Signal and Image Processing - Proceedings of the 12th International Symposium on Mathematical Morphology (ISMM), volume 9082 of Lecture Notes in Computer Science, pages 561-572, Reykjavik, Iceland, 2015. Springer.

30. Jean-Pierre Braquelaire and Luc Brun. Image segmentation with topological maps and inter-pixel representation. Journal of Visual Communication and Image Representation, 9(1):62-79, 1998.

31. Michal Busta, Lukas Neumann, and Jiri Matas. Fastext: Efficient unconstrained scene text detector. In IEEE International Conference on Computer Vision, pages 12061214. IEEE, 2015.

32. Thomas Caissard, David Coeurjolly, Jacques-Olivier Lachaud, and Tristan Roussillon. Heat kernel LaplaceBeltrami operator on digital surfaces. In International Conference on Discrete Geometry for Computer Imagery, pages 241-253. Springer, 2017.
33. Vicent Caselles and Pascal Monasse. Grain filters. Journal of Mathematical Imaging and Vision, 17(3):249-270, 2002.

34. Vicent Caselles and Pascal Monasse. Geometric description of images as topographic maps, ser. Lecture Notes in Mathematics. Springer-Verlag, 1984, 2009.

35. Thomas Christopher Cecil. Numerical methods for partial differential equations involving discontinuities. PhD thesis, University of California Los Angeles, 2003.

36. Li Chen. Genus computing for 3D digital objects: Algorithm and implementation. arXiv preprint arXiv:0912.4936, 2009.

37. Li Chen. Algorithms for computing topological invariants in $2 \mathrm{D}$ and $3 \mathrm{D}$ digital spaces. arXiv preprint arXiv:1309.4109, 2013.

38. Adam Coates, Blake Carpenter, Carl Case, Sanjeev Satheesh, Bipin Suresh, Tao Wang, David J Wu, and Andrew Y Ng. Text detection and character recognition in scene images with unsupervised feature learning. In International Conference on Document Analysis and Recognition, pages 440-445. IEEE, 2011.

39. Jean Cousty and Gilles Bertrand. Uniqueness of the perfect fusion grid on $\mathbb{Z}^{d}$. Journal of Mathematical Imaging and Vision, 34(3):291-306, 2009.

40. Jean Cousty, Gilles Bertrand, Michel Couprie, and Laurent Najman. Fusion graphs: Merging properties and watersheds. Journal of Mathematical Imaging and Vision, 30(1):87-104, January 2008.

41. Jean Cousty, Michel Couprie, Laurent Najman, and Gilles Bertrand. Weighted fusion graphs: merging properties and watersheds. Discrete Applied Mathematics, 156(15):30113027, 2008.

42. Sébastien Crozet and Thierry Géraud. A first parallel algorithm to compute the morphological tree of shapes of $n \mathrm{D}$ images. In Proceedings of the 21st IEEE International Conference on Image Processing (ICIP), pages 2933-2937, Paris, France, 2014.

43. Xavier Daragon. Surfaces discrètes et frontières d'objets dans les ordres. PhD thesis, Université de Marne-la-Vallée, 2005.

44. Xavier Daragon, Michel Couprie, and Gilles Bertrand. Marching chains algorithm for Alexandroff-Khalimsky spaces. In International Symposium on Optical Science and Technology, pages 51-62. International Society for Optics and Photonics, 2002.

45. Xavier Daragon, Michel Couprie, and Gilles Bertrand. Discrete surfaces and frontier orders. Journal of Mathematical Imaging and Vision, 23(3):379-399, 2005.

46. Mathieu Desbrun, Mark Meyer, Peter Schröder, and Alan H Barr. Discrete differential-geometry operators in nd. preprint, the Caltech Multi-Res Modeling Group, 2000.

47. Marc Pierrot Deseilligny, Georges Stamon, and Ching Y Suen. Veinerization: A new shape description for flexible skeletonization. IEEE Transactions on Pattern Analysis and Machine Intelligence, 20(5):505-521, 1998.

48. Tamal K Dey and Sumanta Guha. Computing homology groups of simplicial complexes in $\mathbb{R}^{3}$. Journal of the ACM, 45(2):266-287, 1998.

49. Juan Luis Diaz-de Leon and Juan Humberto SossaAzuela. On the computation of the Euler number of a binary object. Pattern Recognition, 29(3):471-476, 1996.

50. Richard O Duda and John H Munson. Graphical-dataprocessing research study and experimental investigation. Technical report, DTIC Document, 1967.

51. Charles R Dyer. Computing the Euler number of an image from its quadtree. Computer graphics and image processing, 13(3):270-276, 1980 . 
52. Ulrich Eckhardt and Longin Jan Latecki. Digital topology. Institut für Angewandte Mathematik, 1994.

53. Herbert Edelsbrunner, David Letscher, and Afra Zomorodian. Topological persistence and simplification. In Foundations of Computer Science, 2000. Proceedings. 41st Annual Symposium on, pages 454-463. IEEE, 2000.

54. Boris Epshtein, Eyal Ofek, and Yonatan Wexler. Detecting text in natural scenes with stroke width transform. In IEEE Conference on Computer Vision and Pattern Recognition, pages 2963-2970. IEEE, 2010.

55. Alexander V Evako, Ralph Kopperman, and Yurii V Mukhin. Dimensional properties of graphs and digital spaces. Journal of Mathematical Imaging and Vision, 6(23):109-119, 1996.

56. Jonathan Fabrizio, Myriam Robert-Seidowsky, Séverine Dubuisson, Stefania Calarasanu, and Raphaël Boissel. Textcatcher: A method to detect curved and challenging text in natural scenes. International Journal on Document Analysis and Recognition, 19(2):99-117, 2016.

57. Sylvain Faisan, Nicolas Passat, Vincent Noblet, Renée Chabrier, and Christophe Meyer. Topology preserving warping of 3D binary images according to continuous oneto-one mappings. IEEE Transactions on Image Processing, 20(8):2135-2145, 2011.

58. Evgraf Stepanovitch Fedorov. Course of crystallography. Published by RK Rikker, Saint-Petersburg (in Russian), 1901.

59. Christophe Fiorio. Approche interpixel en analyse d'images, une topologie et des algorithmes de segmentation. PhD thesis, Technische Universität Wien, 1995.

60. Christophe Fiorio. A topologically consistent representation for image analysis: The frontiers topological graph. In Discrete Geometry for Computer Imagery, pages 151-162. Springer, 1996.

61. Robin Forman. Morse theory for cell complexes. Advances in Mathematics, 134(1):90-145, 1998.

62. Thierry Géraud, Edwin Carlinet, and Sébastien Crozet. Self-duality and discrete topology: Links between the morphological tree of shapes and well-composed gray-level images. In J.A. Benediktsson, J. Chanussot, L. Najman, and H. Talbot, editors, Mathematical Morphology and Its Application to Signal and Image Processing - Proceedings of the 12th International Symposium on Mathematical Morphology (ISMM), volume 9082 of Lecture Notes in Computer Science, pages 573-584, Reykjavik, Iceland, 2015. Springer.

63. Thierry Géraud, Edwin Carlinet, Sébastien Crozet, and Laurent Najman. A quasi-linear algorithm to compute the tree of shapes of $n$-D images. In C.L. Luengo Hendriks, G. Borgefors, and R. Strand, editors, Mathematical Morphology and Its Application to Signal and Image Processing - Proceedings of the 11th International Symposium on Mathematical Morphology (ISMM), volume 7883 of Lecture Notes in Computer Science, pages 98-110, Uppsala, Sweden, 2013. Springer.

64. Thierry Géraud, Yongchao Xu, Edwin Carlinet, and Nicolas Boutry. Introducing the Dahu pseudo-distance. In J. Angulo, S. Velasco-Forero, and F. Meyer, editors, Mathematical Morphology and Its Application to Signal and Image Processing - Proceedings of the 13th International Symposium on Mathematical Morphology (ISMM), volume 10225 of Lecture Notes in Computer Science, pages 55-67, Fontainebleau, France, May 2017. Springer.

65. Rocío González-Díaz, María José Jiménez, and Belén Medrano. Cohomology ring of 3D cubical complexes. In IWCIA Special Track on Applications, pages 139-150, 2009.
66. Rocío González-Díaz, María José Jiménez, and Belén Medrano. Cubical cohomology ring of 3D photographs. International Journal of Imaging Systems and Technology, 21(1):76-85, 2011.

67. Rocío González-Díaz, María José Jiménez, and Belén Medrano. Well-composed cell complexes. In Discrete Geometry for Computer Imagery, volume 6607 of Lecture Notes in Computer Science, pages 153-162. Springer, 2011.

68. Rocío González-Díaz, María José Jiménez, and Belén Medrano. 3D well-composed polyhedral complexes. Discrete Applied Mathematics, 183:59-77, 2015.

69. Rocío González-Díaz, María José Jiménez, and Belén Medrano. Encoding specific 3D polyhedral complexes using 3D binary images. In Discrete Geometry for Computer Imagery, pages 268-281. Springer, 2016.

70. Rocío González-Díaz, Javier Lamar, and Ronald Umble. Cup products on polyhedral approximations of 3D digital images. In International Workshop on Combinatorial Image Analysis, pages 107-119. Springer, 2011.

71. Rocío González-Díaz and Pedro Real. Towards digital cohomology. In Discrete Geometry for Computer Imagery, volume 2886 of Lecture Notes in Computer Science, pages 92-101. Springer, 2003.

72. Rocío González-Díaz and Pedro Real. On the cohomology of 3D digital images. Discrete Applied Mathematics, 147(2):245-263, 2005.

73. Leo J Grady and Jonathan R Polimeni. Discrete calculus: Applied analysis on graphs for computational science. Springer Science \& Business Media, 2010.

74. Stephen B Gray. Local properties of binary images in two dimensions. IEEE Transactions on Computers, 100(5):551-561, 1971.

75. Marvin J Greenberg. Lectures on algebraic topology, volume 9. WA Benjamin New York, 1967.

76. Ari Gross and Longin Jan Latecki. Digitizations preserving topological and differential geometric properties. Computer Vision and Image Understanding, 62(3):370381, 1995.

77. Xiao Han, Chenyang Xu, and Jerry L Prince. A topology preserving deformable model using level sets. In IEEE Conference on Computer Vision and Pattern Recognition, volume 2, pages 765-770. IEEE, 2001.

78. Xiao Han, Chenyang Xu, and Jerry L Prince. A topology preserving level set method for geometric deformable models. IEEE Transactions on Pattern Analysis and Machine Intelligence, 25(6):755-768, 2003.

79. Henk Heijmans. Theoretical aspects of gray-level morphology. IEEE Transactions on Pattern Analysis and Machine Intelligence, 13(6):568-582, 1991.

80. Henk Heijmans. Morphological image operators, volume 25 of Advances in Electronics and Electron Physics. Academic Press, 1994.

81. Lê Duy Huỳnh, Yongchao $\mathrm{Xu}$, and Thierry Géraud. Morphology-based hierarchical representation with application to text segmentation in natural images. In Proceedings of the 23rd International Conference on Pattern Recognition (ICPR), pages 4029-4034, Cancun, Mexico, December 2016. IEEE Computer Society.

82. Ludvik Janos and Azriel Rosenfeld. Digital connectedness: An algebraic approach. Pattern Recognition Letters, 1(3):135-139, 1983.

83. C. Jordan. Cours d'Analyse de l'Ecole Polytechnique, volume 3. Gauthier-Villars, Paris, 1887.

84. Tao Ju, Frank Losasso, Scott Schaefer, and Joe Warren. Dual contouring of Hermite data. In ACM Transactions on Graphics, volume 21(3), pages 339-346. ACM, 2002. 
85. John L Kelley. General topology. the university series in higher mathematics, 1955.

86. Efim Khalimsky. Applications of connected ordered topological spaces in topology. Conference of Math, Department of Povolosia, 1970.

87. Efim Khalimsky. Ordered Topological Spaces. Naukova Dumka Press, Kiev, 1977.

88. Efim Khalimsky, Ralph Kopperman, and Paul R Meyer. Computer graphics and connected topologies on finite ordered sets. Topology and its Applications, 36(1):1-17, 1990.

89. Robert M Kirby, Valerio Pascucci, Cláudio T Silva, Thomas J Peters, Julien Tierny, Carlos Scheidegger, L Gustavo Nonato, and Tiago Etiene. Topology verification for isosurface extraction. IEEE Transactions on $V i$ sualization and Computer Graphics, 18(6):952-965, 2012.

90. T Yung Kong and Azriel Rosenfeld. Digital topology: Introduction and survey. Computer Vision, Graphics, and Image Processing, 48(3):357-393, 1989.

91. T Yung Kong and Azriel Rosenfeld. If we use 4-or 8connectedness for both the objects and the background, the Euler characteristics is not locally computable. Pattern Recognition Letters, 11(4):231-232, 1990.

92. Ralph Kopperman. The Khalimsky line as a foundation for digital topology. In YL. O, A. Toet, D. Foster H.J.A.M. Heijmans, and P. Meer, editors, Shape in Picture, volume 126 of Computer and Systems Sciences, pages 3-20. Springer, 1994.

93. Ralph Kopperman, Paul R Meyer, and Richard G Wilson. A Jordan surface theorem for three-dimensional digital spaces. Discrete \& Computational Geometry, 6(2):155161, 1991.

94. Ullrich Köthe. Generische Programmierung für die Bildverarbeitung. BoD-Books on Demand, 2000.

95. Vladimir Kovalevsky. Axiomatic digital topology. Journal of Mathematical Imaging and Vision, 26(1):41-58, 2006.

96. Vladimir A Kovalevsky. Finite topology as applied to image analysis. Computer Vision, Graphics, and Image Processing, 46(2):141-161, 1989.

97. Nils Krahnstoever and Cristian Lorenz. Computing curvature-adaptive surface triangulations of threedimensional image data. The Visual Computer, 20(1):17$36,2004$.

98. Jacques-Olivier Lachaud and Annick Montanvert. Continuous analogs of digital boundaries: A topological approach to iso-surfaces. Graphical Models and Image Processing, 62(3):129-164, 2000.

99. Jacques-Olivier Lachaud and Boris Thibert. Properties of gauss digitized shapes and digital surface integration. Journal of Mathematical Imaging and Vision, 54(2):162$180,2016$.

100. Longin Jan Latecki. 3D well-composed pictures. Graphical Models and Image Processing, 59(3):164-172, 1997.

101. Longin Jan Latecki. Discrete representation of spatial objects in computer vision, volume 11. Springer Science \& Business Media, 1998.

102. Longin Jan Latecki. Well-composed sets. Advances in Electronics and Electron Physics, 112:95-163, 2000.

103. Longin Jan Latecki, Christopher Conrad, and Ari Gross. Preserving topology by a digitization process. Journal of Mathematical Imaging and Vision, 8(2):131-159, 1998.

104. Longin Jan Latecki, Ulrich Eckhardt, and Azriel Rosenfeld. Well-composed sets. Computer Vision and Image Understanding, 61(1):70-83, 1995.

105. Carole Le Guyader and Luminita A Vese. Self-repelling snakes for topology-preserving segmentation models. IEEE Transactions on Image Processing, 17(5):767-779, 2008.
106. Chung-Nim Lee, Timothy Poston, and Azriel Rosenfeld. Winding and Euler numbers for 2D and 3D digital images. Graphical Models and Image Processing, 53(6):522-537, 1991.

107. Chung-Nim Lee and Azriel Rosenfeld. Computing the Euler number of a 3D image. Center for Automation Research Technical Report CAR-TR-205, 1986.

108. Jung-Jin Lee, Pyoung-Hean Lee, Seong-Whan Lee, Alan L Yuille, and Christof Koch. Adaboost for text detection in natural scene. In ICDAR, pages 429-434, 2011.

109. Bruno Lévy. Laplace-Beltrami eigenfunctions towards an algorithm that" understands" geometry. In Shape Modeling and Applications, 2006. SMI 2006. IEEE International Conference on, pages 13-13. IEEE, 2006.

110. Elon L Lima. The Jordan-Brouwer separation theorem for smooth hypersurfaces. The American Mathematical Monthly, 95(1):39-42, 1988.

111. Jimin Liu, Su Huang, and Wieslaw L Nowinski. Registration of brain atlas to $\mathrm{mr}$ images using topology preserving front propagation. Journal of Signal Processing Systems, 55(1-3):209-216, 2009.

112. William E Lorensen and Harvey E Cline. Marching cubes: A high resolution 3D surface construction algorithm. In Special Interest Group on Computer GRAPHics and Interactive Techniques, volume 21(3), pages 163-169. ACM, 1987.

113. Wolfram HHJ Lunscher and Michael P Beddoes. Fast binary-image boundary extraction. Computer Vision, Graphics, and Image Processing, 38(3):229-257, 1987.

114. Jean-François Mangin, Vincent Frouin, Isabelle Bloch, Jean Régis, and Jaime López-Krahe. From 3d magnetic resonance images to structural representations of the cortex topography using topology preserving deformations. Journal of Mathematical Imaging and Vision, 5(4):297318, 1995.

115. Jocelyn Marchadier, Didier Arquès, and Sylvain Michelin. Thinning grayscale well-composed images. Pattern Recognition Letters, 25(5):581-590, 2004.

116. Loïc Mazo. Déformations homotopiques dans les images digitales n-aires. $\mathrm{PhD}$ thesis, Université de Strasbourg, 2011.

117. Loïc Mazo, Nicolas Passat, Michel Couprie, and Christian Ronse. Digital imaging: A unified topological framework. Journal of Mathematical Imaging and Vision, 44(1):1937, 2012.

118. Loïc Mazo, Nicolas Passat, Michel Couprie, and Christian Ronse. Topology on digital label images. Journal of Mathematical Imaging and Vision, 44(3):254-281, 2012.

119. Enric Meinhardt-Llopis. Morphological and statistical techniques for the analysis of $3 D$ images. PhD thesis, Universitat Pompeu Fabra, Spain, 2011.

120. Fernand Meyer. Skeletons and perceptual graphs. Signal Processing, 16(4):335-363, 1989.

121. Mark Meyer, Mathieu Desbrun, Peter Schröder, and Alan H Barr. Discrete differential-geometry operators for triangulated 2-manifolds. In H.C. Hege and K. Polthier, editors, Visualization and mathematics, volume 3, pages 35-57. Springer, 2003.

122. Sanae Miri, Nicolas Passat, and Jean-Paul Armspach. Topology-preserving discrete deformable model: Application to multi-segmentation of brain mri. In International Conference on Image and Signal Processing, pages 67-75. Springer, 2008.

123. Pascal Monasse and Frederic Guichard. Fast computation of a contrast-invariant image representation. IEEE Transactions on Image Processing, 9(5):860-872, 2000. 
124. John P Mylopoulos and Theodosios Pavlidis. On the topological properties of quantized spaces, I. The notion of dimension. Journal of the ACM, 18(2):239-246, 1971.

125. John P Mylopoulos and Theodosios Pavlidis. On the topological properties of quantized spaces, II. Connectivity and order of connectivity. Journal of the ACM, 18(2):247-254, 1971.

126. Laurent Najman and Michel Couprie. Watershed algorithms and contrast preservation. In I. Nyström, G. Sanniti di Baja, and S. Svensson, editors, Discrete Geometry for Computer Imagery, volume 2886 of Lecture Notes in Computer Science, pages 62-71. Springer, 2003.

127. Laurent Najman and Thierry Géraud. Discrete set-valued continuity and interpolation. In C.L. Luengo Hendriks, G. Borgefors, and R. Strand, editors, Mathematical Morphology and Its Application to Signal and Image Processing - Proceedings of the 11th International Symposium on Mathematical Morphology (ISMM), volume 7883 of Lecture Notes in Computer Science, pages 37-48, Heidelberg, 2013. Springer.

128. Laurent Najman and Pascal Romon, editors. Discrete curvature: theory and applications, volume 3 of Actes des rencontres du CIRM, France, December 2014. CEDRAM.

129. Laurent Najman and Pascal Romon, editors. Modern Approaches to Discrete Curvature, volume 2184 of Lecture Notes in Mathematics. Springer International Publishing, 2017.

130. Laurent Najman and Michel Schmitt. Watershed of a continuous function. Signal Processing, 38(1):99-112, 1994.

131. Laurent Najman and Hugues Talbot. Mathematical Morphology. John Wiley \& Sons, 2013.

132. Mikio Nakahara. Geometry, topology and physics. CRC Press, 2003.

133. Maxwell Herman Alexander Newman. Elements of the topology of plane sets of points. Cambridge, 1939.

134. Phuc Ngo, Yukiko Kenmochi, Nicolas Passat, and Hugues Talbot. Combinatorial structure of rigid transformations in 2D digital images. Computer Vision and Image Understanding, 117(4):393-408, 2013.

135. Phuc Ngo, Yukiko Kenmochi, Nicolas Passat, and Hugues Talbot. Sufficient conditions for topological invariance of $2 \mathrm{D}$ images under rigid transformations. In Discrete Geometry for Computer Imagery, volume 7749 of Lecture Notes in Computer Science, pages 155-168. Springer, 2013.

136. Phuc Ngo, Yukiko Kenmochi, Nicolas Passat, and Hugues Talbot. Topology-preserving conditions for $2 \mathrm{~d}$ digital images under rigid transformations. Journal of mathematical imaging and vision, 49(2):418-433, 2014.

137. Phuc Ngo, Nicolas Passat, Yukiko Kenmochi, and Hugues Talbot. Well-composed images and rigid transformations. In IEEE International Conference on Image Processing, pages 3035-3039, 2013.

138. Phuc Ngo, Nicolas Passat, Yukiko Kenmochi, and Hugues Talbot. Topology-preserving rigid transformation of 2D digital images. IEEE Transactions on Image Processing, 23(2):885-897, 2014

139. Seymour Papert and Marvin Minsky. Perceptrons: An Introduction to Computational Geometry. The MIT Press, 1969.

140. Theodosios Pavlidis. Algorithms for Graphics and Image Processing. Springer Science \& Business Media, 2012.

141. Dzung L Pham, Pierre-Louis Bazin, and Jerry L Prince. Digital topology in brain imaging. Signal Processing Magazine, 27(4):51-59, 2010.

142. Fabrice Poupon, J Mangin, Dominique Hasboun, Cyril Poupon, I Magnin, and Vincent Frouin. Multi-object deformable templates dedicated to the segmentation of brain deep structures. Medical Image Computing and Computer-Assisted Intervention, MICCAI '98, pages 1134-1143, 1998

143. Jos BTM Roerdink and Arnold Meijster. The watershed transform: Definitions, algorithms and parallelization strategies. Fundamenta Informaticae, 41(1-2):187$228,2000$.

144. Christian Ronse. Flat morphological operators on arbitrary power lattices. In T. Asano, R. Klette, and C. Ronse, editors, Geometry, Morphology, and Computational Imaging, volume 2616 of Lecture Notes in Computer Science, pages 1-21. Springer, 2003.

145. Azriel Rosenfeld. Connectivity in digital pictures. Journal of the ACM, 17(1):146-160, 1970.

146. Azriel Rosenfeld. Arcs and curves in digital pictures. Journal of the ACM, 20(1):81-87, 1973.

147. Azriel Rosenfeld. Adjacency in digital pictures. Information and Control, 26(1):24-33, 1974.

148. Azriel Rosenfeld. Digital topology. American Mathematical Monthly, 86(8):621-630, 1979.

149. Azriel Rosenfeld. Fuzzy digital topology. Information and Control, 40(1):76-87, 1979.

150. Azriel Rosenfeld. Picture Languages-Formal Model of Picture Recognition. Academic Press, New York, 1979.

151. Azriel Rosenfeld. On connectivity properties of grayscale pictures. Pattern Recognition, 16(1):47-50, 1983.

152. Azriel Rosenfeld, T Yung Kong, and Akira Nakamura. Topology-preserving deformations of two-valued digital pictures. Graphical Models and Image Processing, 60(1):24-34, 1998.

153. Azriel Rosenfeld and John L Pfaltz. Sequential operations in digital picture processing. Journal of the ACM, 13(4):471-494, 1966

154. Raif M Rustamov. Laplace-Beltrami eigenfunctions for deformation invariant shape representation. In Proceedings of the fifth Eurographics symposium on Geometry processing, pages 225-233. Eurographics Association, 2007.

155. Punam K Saha, Robin Strand, and Gunilla Borgefors. Digital topology and geometry in medical imaging: A survey. IEEE Transactions on Medical Imaging, 34(9):19401964, 2015.

156. Philippe Salembier and Jean Serra. Flat zones filtering, connected operators, and filters by reconstruction. IEEE Transactions on Image Processing, 4(8):1153-1160, 1995.

157. Florent Ségonne. Active contours under topology control - genus preserving level sets. International Journal of Computer Vision, 79(2):107-117, 2008.

158. Jean Serra. Image Analysis and Mathematical Morphology, Vol. 1. Academic Press, 1982.

159. Jean Serra and B Ravi Kiran. Digitization of partitions and tessellations. In N. Normand, J. Guédon, and F. Autrusseau, editors, Discrete Geometry for Computer Imagery, volume 9647 of Lecture Notes in Computer Science, pages 323-334. Springer, 2016.

160. Raj Shekhar, Elias Fayyad, Roni Yagel, and J Fredrick Cornhill. Octree-based decimation of marching cubes surfaces. In Visualization'96, pages 335-342. IEEE, 1996.

161. Yonggang Shi, Rongjie Lai, Raja Gill, Daniel Pelletier, David Mohr, Nancy Sicotte, and Arthur W Toga. Conformal metric optimization on surface (CMOS) for deformation and mapping in Laplace-Beltrami embedding space. In International Conference on Medical Image Computing and Computer-Assisted Intervention, pages 327-334. Springer, 2011.

162. Marcelo Siqueira, Longin Jan Latecki, and Jean Gallier. Making 3D binary digital images well-composed. In Electronic Imaging 2005, pages 150-163. International Society for Optics and Photonics, 2005. 
163. Marcelo Siqueira, Longin Jan Latecki, Nicholas Tustison, Jean Gallier, and James Gee. Topological repairing of 3D digital images. Journal of Mathematical Imaging and Vision, 30(3):249-274, 2008.

164. Lauro Snidaro and Gian Luca Foresti. Real-time thresholding with Euler numbers. Pattern Recognition Letters, 24(9):1533-1544, 2003.

165. Pierre Soille. Morphological Image Analysis: Principles and Applications. Springer Science \& Business Media, 2013.

166. Pierre Soille and Martino Pesaresi. Advances in mathematical morphology applied to geoscience and remote sensing. IEEE Transactions on Geoscience and Remote Sensing, 40(9):2042-2055, 2002.

167. Juan Humberto Sossa-Azuela, R Santiago-Montero, M Pérez-Cisneros, and E Rubio-Espino. Computing the Euler number of a binary image based on a vertex codification. Journal of Applied Research and Technology, 11(3):360-370, 2013.

168. Peer Stelldinger. Image Digitization and its Influence on Shape Properties in Finite Dimensions, volume 312. IOS Press, 2008.

169. Peer Stelldinger and Ullrich Köthe. Towards a general sampling theory for shape preservation. Image and Vision Computing, 23(2):237-248, 2005.

170. Peer Stelldinger and Longin Jan Latecki. 3D object digitization: Majority interpolation and marching cubes. In IEEE International Conference on Pattern Recognition, volume 2, pages 1173-1176. IEEE, 2006.

171. Peer Stelldinger, Longin Jan Latecki, and Marcelo Siqueira. Topological equivalence between a $3 \mathrm{D}$ object and the reconstruction of its digital image. IEEE Transactions on Pattern Analysis and Machine Intelligence, 29(1):126140, 2007.

172. Peer Stelldinger and Robin Strand. Topology preserving digitization with FCC and $\mathrm{BCC}$ grids. In International Workshop on Combinatorial Image Analysis, pages 226240. Springer, 2006.

173. Lawrence Neff Stout. Two discrete forms of the Jordan curve theorem. The American Mathematical Monthly, 95(4):332-336, 1988.

174. Ganesh Sundaramoorthi and Anthony Yezzi. Global regularizing flows with topology preservation for active contours and polygons. IEEE Transactions on Image Processing, 16(3):803-812, 2007.

175. Mohamed Tajine and Christian Ronse. Topological properties of Hausdorff discretizations. In J. Goutsias, L. Vincent, and D.S. Bloomberg, editors, Mathematical Morphology and its Applications to Image and Signal Processing, volume 18 of Computational Imaging and Vision, pages 41-50. Springer, 2002.

176. Junichiro Toriwaki and Hiroyuki Yoshida. Fundamentals of three-dimensional digital image processing. Springer Science \& Business Media, 2009.

177. Wei-Chih Tu, Shengfeng He, Qingxiong Yang, and ShaoYi Chien. Real-time salient object detection with a minimum spanning tree. In Proceedings of the IEEE Conference on Computer Vision and Pattern Recognition, pages 2334-2342, 2016.

178. Nicholas J Tustison, Brian B Avants, Marcelo Siqueira, and James C Gee. Topological well-composedness and glamorous glue: A digital gluing algorithm for topologically constrained front propagation. IEEE Transactions onImage Processing, 20(6):1756-1761, 2011.

179. Helge Tverberg. A proof of the Jordan curve theorem. Bulletin of the London Mathematical Society, 12(1):34-38, 1980.
180. Lucas J Van Vliet, Ian T Young, and Guus L Beckers. An edge detection model based on non-linear Laplace filtering. In International workshop on Pattern Recognition and Artificial Intelligence, towards an Integration, 1988.

181. K Voss. Images, objects, and surfaces in $\mathbb{Z}^{n}$. International Journal of Pattern Recognition and Artificial Intelligence, 5(05):797-808, 1991.

182. Tao Wang, David J Wu, Adam Coates, and Andrew Y $\mathrm{Ng}$. End-to-end text recognition with convolutional neural networks. In International Conference on Pattern Recognition, pages 3304-3308. IEEE, 2012.

183. Yang Wang and Prabir Bhattacharya. Digital connectivity and extended well-composed sets for gray images. Computer Vision and Image Understanding, 68(3):330345, 1997.

184. Paul R Wilson. Euler formulas and geometric modeling. IEEE Computer Graphics and Applications, 8(5):24-36, 1985.

185. Yongchao Xu, Edwin Carlinet, Thierry Géraud, and Laurent Najman. Efficient computation of attributes and saliency maps on tree-based image representations. In J.A. Benediktsson, J. Chanussot, L. Najman, and H. Talbot, editors, Mathematical Morphology and Its Application to Signal and Image Processing - Proceedings of the 12th International Symposium on Mathematical Morphology (ISMM), volume 9082 of Lecture Notes in Computer Science, pages 693-704, Reykjavik, Iceland, 2015. Springer.

186. Yongchao Xu, Edwin Carlinet, Thierry Géraud, and Laurent Najman. Hierarchical segmentation using tree-based shape spaces. IEEE Transactions on Pattern Analysis and Machine Intelligence, 39(3):457-469, April 2017.

187. Yongchao $\mathrm{Xu}$, Thierry Géraud, and Laurent Najman. Context-based energy estimator: Application to object segmentation on the tree of shapes. In Proceedings of the 19th IEEE International Conference on Image Processing (ICIP), pages 1577-1580, Orlando, Florida, USA, October 2012.

188. Yongchao Xu, Thierry Géraud, and Laurent Najman. Morphological filtering in shape spaces: Applications using tree-based image representations. In Proceedings of the 21st International Conference on Pattern Recognition $(I C P R)$, pages 485-488, Tsukuba Science City, Japan, November 2012. IEEE Computer Society.

189. Yongchao Xu, Thierry Géraud, and Laurent Najman. Two applications of shape-based morphology: Blood vessels segmentation and a generalization of constrained connectivity. In C.L. Luengo Hendriks, G. Borgefors, and R. Strand, editors, Mathematical Morphology and Its Application to Signal and Image Processing - Proceedings of the 11th International Symposium on Mathematical Morphology (ISMM), volume 7883 of Lecture Notes in Computer Science, pages 390-401, Heidelberg, 2013. Springer.

190. Yongchao Xu, Thierry Géraud, and Laurent Najman. Connected filtering on tree-based shape-spaces. IEEE Transactions on Pattern Analysis and Machine Intelligence, 38(6):1126-1140, June 2016.

191. Yongchao Xu, Pascal Monasse, Thierry Géraud, and Laurent Najman. Tree-based morse regions: A topological approach to local feature detection. IEEE Transactions on Image Processing, 23(12):5612-5625, 2014.

192. Hyun S Yang and Sanjay Sengupta. Intelligent shape recognition for complex industrial tasks. IEEE Control Systems Magazine, 8(3):23-30, 1988.

193. Qixiang Ye and David Doermann. Text detection and recognition in imagery: A survey. IEEE Transactions on 
Pattern Analysis and Machine Intelligence, 37(7):14801500, 2015.

194. Shigeki Yokoi, Jun-Ichiro Toriwaki, and Teruo Fukumura. An analysis of topological properties of digitized binary pictures using local features. Computer Graphics and Image Processing, 4(1):63-73, 1975.

195. Jianming Zhang, Stan Sclaroff, Zhe Lin, Xiaohui Shen, Brian Price, and Radomir Mech. Minimum barrier salient object detection at $80 \mathrm{fps}$. In Proceedings of the IEEE International Conference on Computer Vision, pages 14041412, 2015.

196. Yingying Zhu, Cong Yao, and Xiang Bai. Scene text detection and recognition: Recent advances and future trends. Frontiers of Computer Science, 10(1):19-36, 2016. 